\title{
Video posturography near the limit of stability
}

Citation for published version (APA):

El-Kahky, A. M. (1998). Video posturography near the limit of stability. [Doctoral Thesis, Maastricht University]. Universiteit Maastricht. https://doi.org/10.26481/dis.19981216ae

Document status and date:

Published: 01/01/1998

DOI:

10.26481/dis.19981216ae

Document Version:

Publisher's PDF, also known as Version of record

\section{Please check the document version of this publication:}

- A submitted manuscript is the version of the article upon submission and before peer-review. There can be important differences between the submitted version and the official published version of record.

People interested in the research are advised to contact the author for the final version of the publication, or visit the DOI to the publisher's website.

- The final author version and the galley proof are versions of the publication after peer review.

- The final published version features the final layout of the paper including the volume, issue and page numbers.

Link to publication

\footnotetext{
General rights rights.

- You may freely distribute the URL identifying the publication in the public portal. please follow below link for the End User Agreement:

www.umlib.nl/taverne-license

Take down policy

If you believe that this document breaches copyright please contact us at:

repository@maastrichtuniversity.nl

providing details and we will investigate your claim.
}

Copyright and moral rights for the publications made accessible in the public portal are retained by the authors and/or other copyright owners and it is a condition of accessing publications that users recognise and abide by the legal requirements associated with these

- Users may download and print one copy of any publication from the public portal for the purpose of private study or research.

- You may not further distribute the material or use it for any profit-making activity or commercial gain

If the publication is distributed under the terms of Article $25 \mathrm{fa}$ of the Dutch Copyright Act, indicated by the "Taverne" license above, 


\section{Video posturography near the limit of stability}

Ayman Mohamed EL-Kahky 
Printed by: Unigraphics Maastricht Thesis Universiteit Maastricht

Video posturography near the limit of stability ISBN: 90-9011995-7

NUGI 743

Copyright $\odot 1998$ A.M. EL-Kahky 


\section{Video posturography near the limit of stability}

\section{PROEFSCHRIFT}

ter verkrijging van de graad van doctor aan de Universiteit Maastricht, op gezag van de Rector Magnificus,

Prof. dr. A.C. Nieuwenhuijzen Kruseman volgens het besluit van het College van Decanen, in het openbaar te verdedigen op woensdag 16 december 1998 om 12.00 uur

door

Ayman Mohamed EL-Kahky 


\section{Promotor:}

Prof. dr. J.J. Manni

Co-promotor:

Dr. H. Kingma

\section{Beoordelingscommissie:}

Prof. dr. H. Kuipers (voorzitter)

Dr. W. Bles (TNO Technische Menskunde Soesterberg)

Dr. L.J.J.M. Boumans (Erasmus Universiteit Rotterdam)

Prof. dr. J. Jolles

Prof. dr. J. Troost 


\section{Contents}


Chapter 1 Introduction

Chapter 2 Dynamic posturography in healthy subjects: evaluation of COG measuring techniques and individualisation of test conditions

Chapter 3 Balance control near the limit of stability at various sensory conditions in healthy subjects

Chapter 4 Effect of training on a balance bar upon postural control near the limit of stability in healthy subjects: a double blind placebo controlled study

Chapter 5 Balance control near the limit of stability at various sensory conditions in patients suffering from vertigo or balance disorders

Chapter 6 Body movement strategy near the limit of stability, studied by video-posturography in normal subjects and dizzy patients

Chapter $7 \quad$ General discussion

Summary

Samenvatting

Acknowledgements

Curriculum Vitae 
Chapter 1

\section{General Introduction}


Upright stance has an enormous significance for the human being, because it frees the hands for productive activity (1) and improves visual orientation. Upright stance and balance in general is regulated by neuromuscular control centres in the brain that depend upon redundant information provided by vestibular, visual and proprioceptive input. In the context of balance control these three sensory systems provide the following information. The vestibular system senses absolute head acceleration and spatial orientation. The visual system detects motion and orientation of the image of the visual surround projected on the retina. The proprioceptive system primarily senses the motions of the lower extremities as well as the force exerted by the feet on the support surface. Therefore body orientation is detected in three different frames of references. Vestibular input is partly referenced to gravity, visual input is referenced to the visual surround and proprioceptive input is referenced to an earth based support surface. Absolute orientation to the earth vertical is therefore derived from the vestibular system only, whereas the other senses provide relative orientation references (2). Proprioceptive and visual feedback can be misleading in certain environmental conditions. For example, walking over a surface that moves or changes from a concrete to an irregular soft soil alter the proprioceptive input. Walking through a moving scene with many visual cues generally leads to a more dominant effect of the visual system upon postural control. Upright stance may not be disturbed in such conditions owing to a strategy that focus on the more accurate inputs. Thus balance control depends upon the ability to adaptively modify the relative weighting of each sensory modality, depending upon the environmental context (3).

A balance system abnormality may occur in the three sensory systems mentioned above, in the motor system involved in executing the appropriate musculoskeletal responses and in the associated neuro-muscular and sensory integration centres in the brain (2). The complexity of the balance system (figure 1) makes localisation of any dysequilibrium problem difficult, even if other causes of instability can been ruled out. Loss of balance control seems to be more severe in case of acute loss of vestibular function than in case of sudden impairment of the visual or the proprioceptive system (4). Therefore, loss of balance control has intensively been studied through quantification of both vestibulo-ocular and vestibulo-spinal reflexes. Vestibulo-ocular reflexes often appear to be normal in patients suffering from balance disorders. This might be explained by the fact that the central pathways and muscular systems of the vestibulo-ccular system and the vestibulo-spinal system are distinctive in many aspects $(5,6)$. The adaptive and compensatory mechanisms of both vestibular subsystems also run different courses in case of a sudden peripheral vestibular loss (7). $\mathrm{So}_{3}$ in patients suffering from poor equilibrium, the balance system should be adequately assessed at the vestibulospinal level to establish an adequate diagnosis (8). 


\section{BALANCE}

SENSORS CENTERS

GOAL

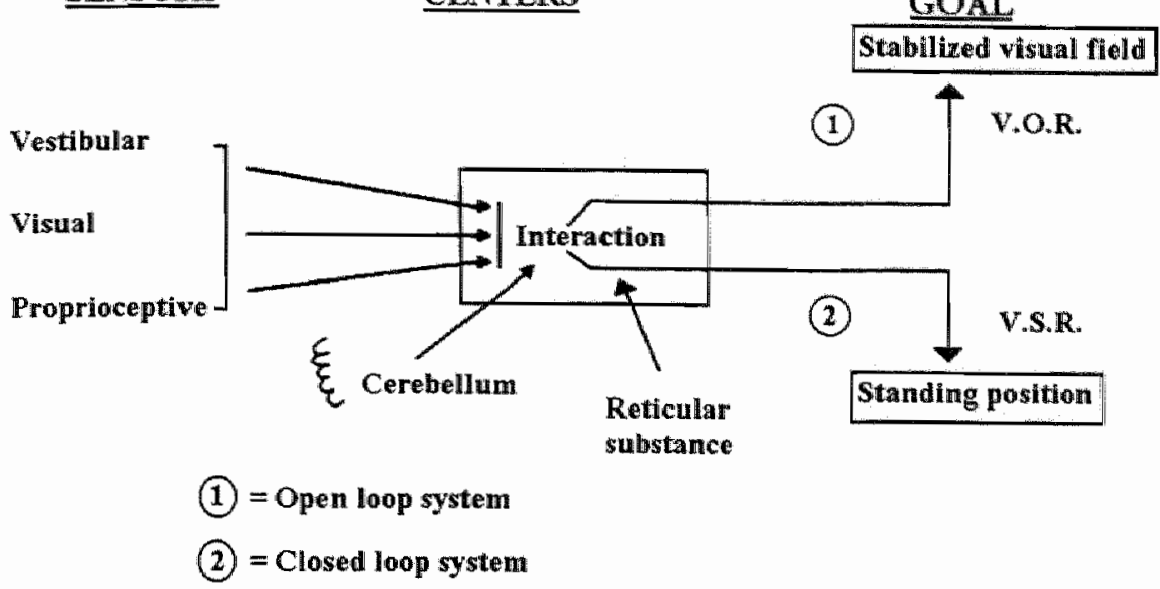

Figure 1. Scheme of balance function indicating the separate pathways of vestibulo ocular (VOR) and vestibulospinal (VSR) reflexes (8).

\section{Stabilometry or static posturography}

Romberg was among the first to recognise the complex sensorimotor interactions required for control of upright stance (9). In this classical test the patient is asked to stand erect, eyes open, the feet slightly separated from each other, and postural control is judged. Romberg originally devised this test to demonstrate postural instability in case of deficits of the posterior column, but was fully aware of the influence of the inputs from vision, labyrinths and general propriocepsis upon postural control: for example, he refined his test by observing the patient's postural sway not only with eyes opened but also with eyes closed (10). Postural sway is considered to reflect the physiological postural muscular activity necessary to keep in balance. This muscular activity induces a continuous to-and-fro movement of the centre of gravity of the body (COG) around the required position $(10,11,12)$. A small (normal) sway is therefore associated with a normal function of the postural control system, whereas a large (abnormal) sway or fall is considered characteristic for deficits anywhere in the postural control system. However the large inter-and intra-individual variability of the test, as well as the large inter-observer variability resulted in a low appreciation of the test as a part of the vestibular test battery. Therefore considerable improvement was expected from the introduction of stabilometry which can be considered as the quantitative version of Romberg's test. Stabilometry became available through the 
development of force platforms that enabled detection and quantification of lateral and anterior-posterior body sway. Here the patient stands on a platform (often called force plate) that is equipped with minimal three force transducers. The transducers detect the vertical forces exerted by the feet on the platform and inform about the force distribution present over the platform. The force distribution changes in case of displacements of the COG. As a consequence detection of the force distribution allows calculation of the absolute position of the vertical projection of the COG on the platform. In this way body sway direction, amplitude and velocity are easily quantified $(1,13)$. Stabilometry (or static posturography) has long been proposed as a relevant tool to examine patients with instability $(10,12,14,15)$. However the poor reproducibility, the low sensitivity and specificity made the clinical relevance of the test questionable.

Many researchers have tried to modify and improve the stabillometry test conditions in order to obtain a relevant clinical tool. Increase of sensitivity can be achieved by placing the feet in front of each other (sensitised Romberg), or by distracting the attention of the subject from the primary task of maintaining balance (Jendrassiks modification), by reducing the proprioceptive information by placing rubber foam on the platform (16), or misleading the visual spatial orientation by placing the platform in a slowly moving visual surround (17). However, the poor reproducibility and low specificity appeared to be consistent and prevented a break through of stabilometry as a rellevant diagnostic tool.

\section{Dynamic posturography}

In a second phase of innovative experiments moving platforms were used to study postural control. This type of posturography is better known as dynamic posturography. The technique was introduced to create a stimulus-response condition or to manipulate the proprioceptive input to the postural control system.

Primary, fast moving platforms (rotatory or translational) were used as stimulators (velocity impulse) to induce postural reflexes, detect movements of body segments and electromyography of postural muscles $(18,41)$. This application proved to be useful in diagnostics of neuromuscular diseases and recently has been reported to contribute substantially to detect deficits of the vestibular system (42).

Secondary, another class of dynamic posturography was introduced: sway referenced platforms were applied to reduce or mislead the proprioceptive input in a sophisticated and computer controlled way in combination with a sway referenced visual surround (19). The specificity of stabilometry was low because the contributions of the visual and vestibular system to the control of posture could not be assessed in comparison to that of the proprioceptive system (20). The new posturography technique proposed was designed to be able to disturb the visual and proprioceptive sensory inputs by a technique called swayreferencing $(10,19)$. The ultimate goal was isolation of one or 
more sensory input systems. In this technique the anterior-posterior movements of the COG of the subject are detected by the platform ${ }^{l}$ and fed into a computer that controls the rotation angle of a tillting platform and a visual surround. Both platform and visual surround, either simultaneously or separately, rotate around an axis through the ankle joints with an angle proportional to the anterior-posterior body sway angle. In this way the orientation of the surface and/or visual surround relative to the body remains constant despite the posterior-anterior body movements suggesting that the subjects spatial orientation to the gravitational vector does not change, whereas the opposite is true. To maintain normal balance, normal subjects will ignore the inaccurate spatial information. This complex dynamic posturography system is commercially available under the name Equitest (21) and has become the standard of posturography worldwide, despite some essential drawbacks that will be discussed here. In this thesis we will refer to this system by the term "the dynamic posturography system according to Nashner and Peters" (22). Through this system a well defined set of six sensory conditions, together known as the sensory organisation test (SOT), are used to evaluate postural control. The patient attempts to maintain upright stance for twenty seconds during the six sensory conditions, and minimises body sway as far as possible. The first two sensory conditions are a standard Romberg test (eyes open and closed on a fixed platform with a fixed visual surround). In condition three (swayreferenced visual surround) the visual surround moves proportional to the body sway and disables visual spatial orientation. In condition four (swayreferenced platform) the platform moves proportional to the anterior-posterior sway and disables proprioceptive orientation of the foot soles and the ankle joints. Condition five is a modification of condition four, whereas the patient is asked to close the eyes: in this condition the proprioceptive orientation is impaired and visual orientation absent. Condition six is combination of condition three and four: both visual and proprioceptive orientation is impaired by a swayreferenced visual surround and swayreferenced platform (figure 2).

In the complete SOT, the patient is exposed to three trials for each of the six conditions. The assessment is based on the assumption that a normal individual can exhibit anterior to posterior sway over a total range of 12.5 degrees without losing balance (23). The equilibrium score is calculated by comparing the angular difference between the patient's calculated maximum anterior to posterior COG displacement (A) (figure $3 a$ ) to this theoretical maximum displacement (B) (figure $3 b$ ). In a normative study Nashner et al (23) showed that B can be estimated to be 12.5 degrees. An equilibrium score of $100 \%$ is perfect (body sway $=0$ ) whereas a score of $0 \%$ is maximum instability and generally results in a fall. In case of a fall the equilibrium score is always set at $0 \%$. The equilibrium score is presented in a bar graph (figure 3c).

\footnotetext{
${ }^{1}$ A fundamental question arises about the measuring techniques applied in almost all dynamic posturographic platforms. During active movement of the platform reaction forces do occur and interfere with the detection of the $\mathrm{COG}$. This phenomenon prevents an accurate detection of body sway (36).
} 


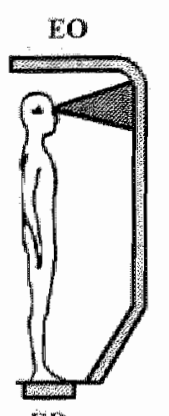

FP

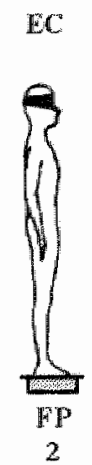

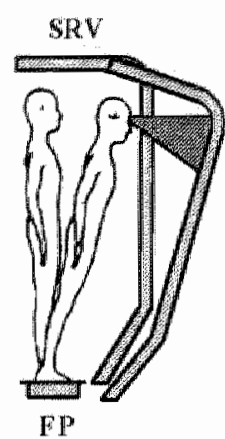

3

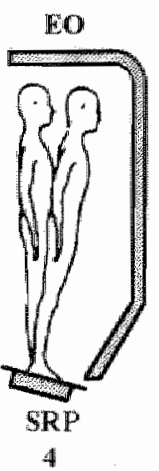

4
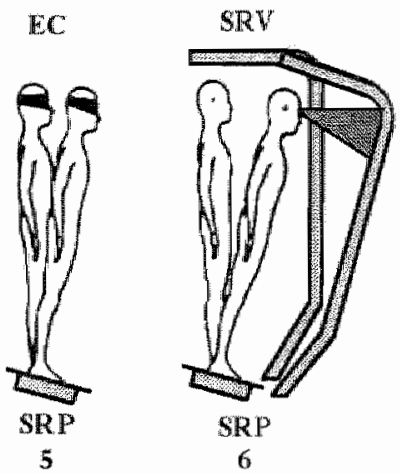

Figure 2. Sensory organisation test: conditions 1 through 6 .

1. Eyes open (EO) and fixed platform (FP), 2. Eyes closed (EC) and fixed platform, 3. Swayreferenced visual surround (SRV). 4. Swayreferenced platform (SRP) and eyes open, 5. Swayreferenced platform and eyes closed, 6. Swayreferenced platform and visual surrourd (22).

Figure 4 shows a typical presentation of the outcome of the complete SOT of the dynamic posturography system according to Nashner and Peters (22). Each sensory condition (numbered 1 to 6 ) is displayed through three black bars representing the equilibrium score of the three successive trials per condition. The broad grey bar displayed at the background of each condition and the composite score (see below) indicates the normative equilibrium score (matched for the subjects age). The composite equilibrium score depicted on the right side of figure 4 is calculated by averaging the three scores for both conditions 1 and 2 , adding these two averaged scores to the equilibrium scores of each trial of sensory conditions $3,4,5$ and 6 , and dividing the sum by 14. Missing trials in conditions 3,4,5 and 6 are replaced by the average score for that condition. The highest possible composite score is 100 .

The SOT has been designed to assess the overall function of the balance control system by manipulating the available sensory information and through verification of the subject"s ability to use various spatial reference frames $(10,16,24,25)$. It is assumed that abnormal equilibrium scores can be observed in case of impairment of one or more of the relevant sensory subsystems or the central integration centres. And indeed, a number of typical SOT patterns have been cescribed and attributed to specific sensory abnormalities $(23,26,27,28,29)$. However these findings have not been confirmed at present. In contrast, Furman (30) concluded that the SOT does not provide localising information in the neurological sense at all. The SOT seems not to provide relevant information regarding the etiology of unilateral vestibular losses either (31). 


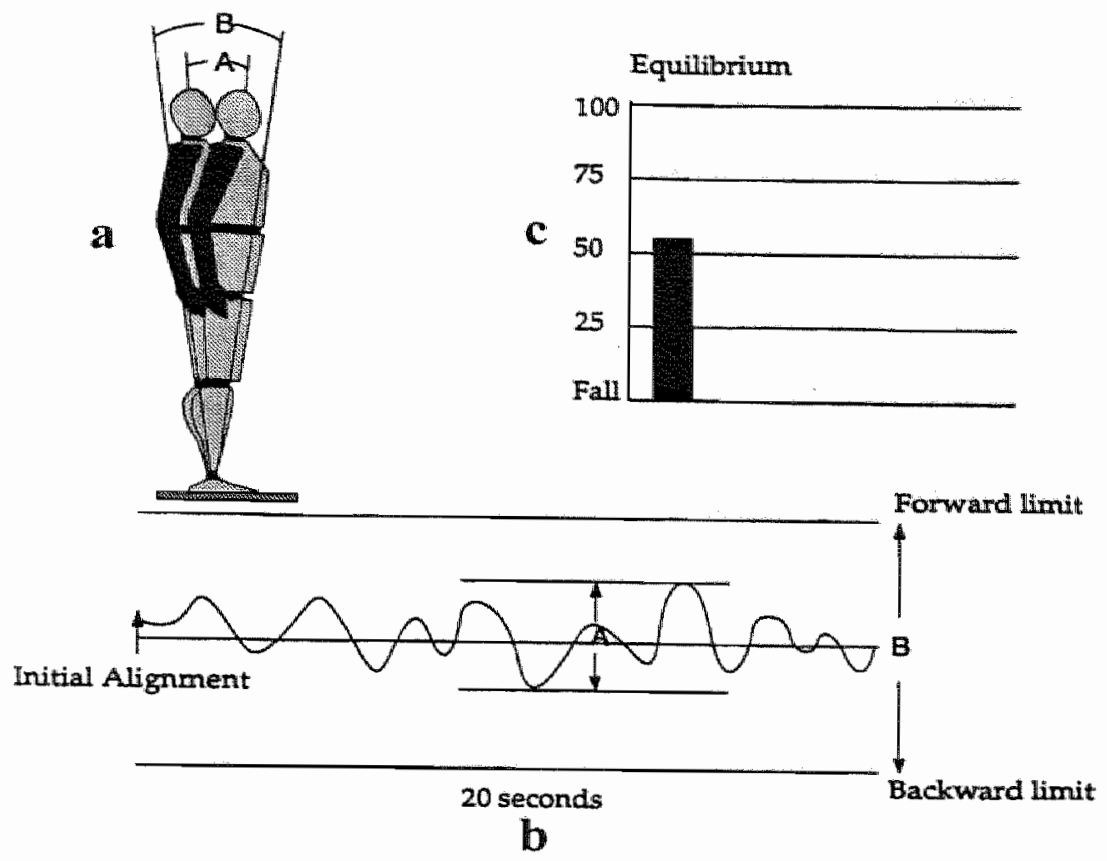

Figure 3a-c. a. visualisation of the anterior-posterior bodysway (A: actual body sway; $B$ : theoretical maximum body sway).

b. body sway as a function of time.

c. the equilibrium score in percent.

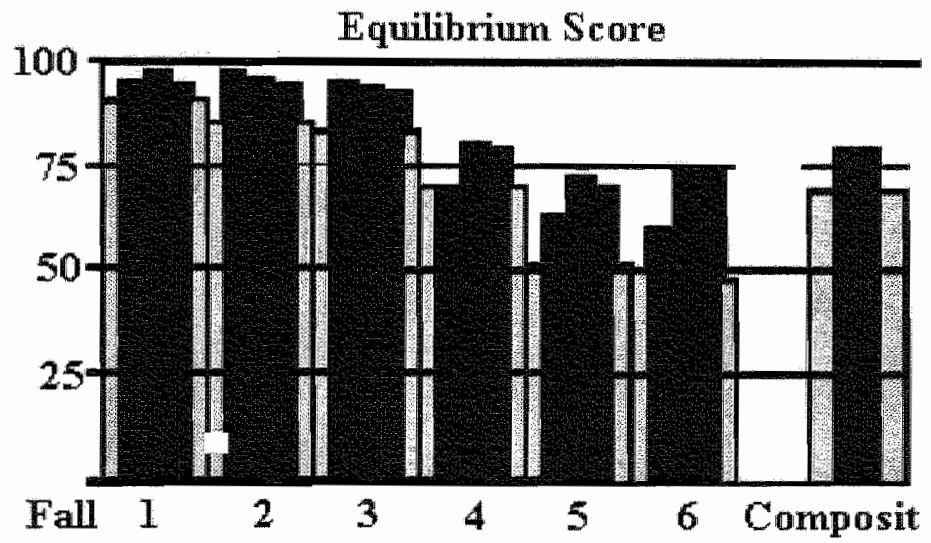

Figure 4. Typical presentation of the test outcome of the SOT according to Nashner and Peters (22). See text for further explanation. 
To our experience the balance score in many patients and normal individuals, is better in the swayreferenced visual surround condition than in the eyes closed condition (Fig. 5). This finding is in contrast to the normal values provided by the commercially available posturography system. Nashner (personal communication, 1995) indicates that the swayreferenced visual surround seems to be more difficult than the closed eyes sensory condition in elderly patients, whereas this effect is less evident in younger subjects. This aspect is quite important, because if conditions 3 and 6 give essentially the same (redundant) information as compared to that obtained through condition 2 and 5 , the experimental set-up could be considerably simplified. It is quite essential to gain more insight in the ability of the SOT to "isolate" the sensory subsystems. The impression exists that the swayreference technique only partly reduces the associated sensory input. At last, postural sway in the lateral plan is of utmost importance with respect to postural control (17) and is completely neglected by the SOT. For the sake of simplicity and due to technical limitations, the work in this thesis had to be restricted to the study of postural sway in the antero-posrerior plane too, but we are fully aware of the fact that balance control should be analysed in two and not in one dimension.

Besides quantification of body sway as a function of the six sensory conditions, Nashner and others (32) also studied body movement strategies used for postural control. They hypothesised that postural control is organised around two basic movement patterns, one using the ankle joint, the other using the hip as centres of rotation $(22,32,33,34)$ (figure $6 \mathrm{a}$ ). Which pattern or combination of patterns is used depends on the size and consistency of the support surface and the position of the COG relative to the limits of stability (22). The basic principle of stable stance is that a subject will not fall as long as the projection of the COG on the ground is within the base of support enclosed by both feet. Through the ankle strategy the COG can be placed forward or backward by rotation around the ankle joints over a maximum angle of approximately 12.5 degrees: in this way the projection of the COG will remain within the base of support (23). The muscles of the feet and the lower limbs have to counteract the forward or backward directed vector of the gravitational force in order to prevent the body from falling. This activation of muscles results in a change of force distribution exerted by the feet on the supporting surface. Therefore the ankle strategy requires a sufficiently large supporting surface. Balancing on a bar or on a soft surface requires another strategy. The ankle strategy is relatively slow because the point of rotation (and the effector) is at a relatively large distance from the COG. The hip strategy relies on torsional movements about the hips in order to rotate the ankle and hip joints in opposite directions and to maintain the $C O G$ at a more or less constant position (23). Using this strategy, the force distribution of the feet exerted upon the supporting surface does not change substantially because the COG hardly moves. 

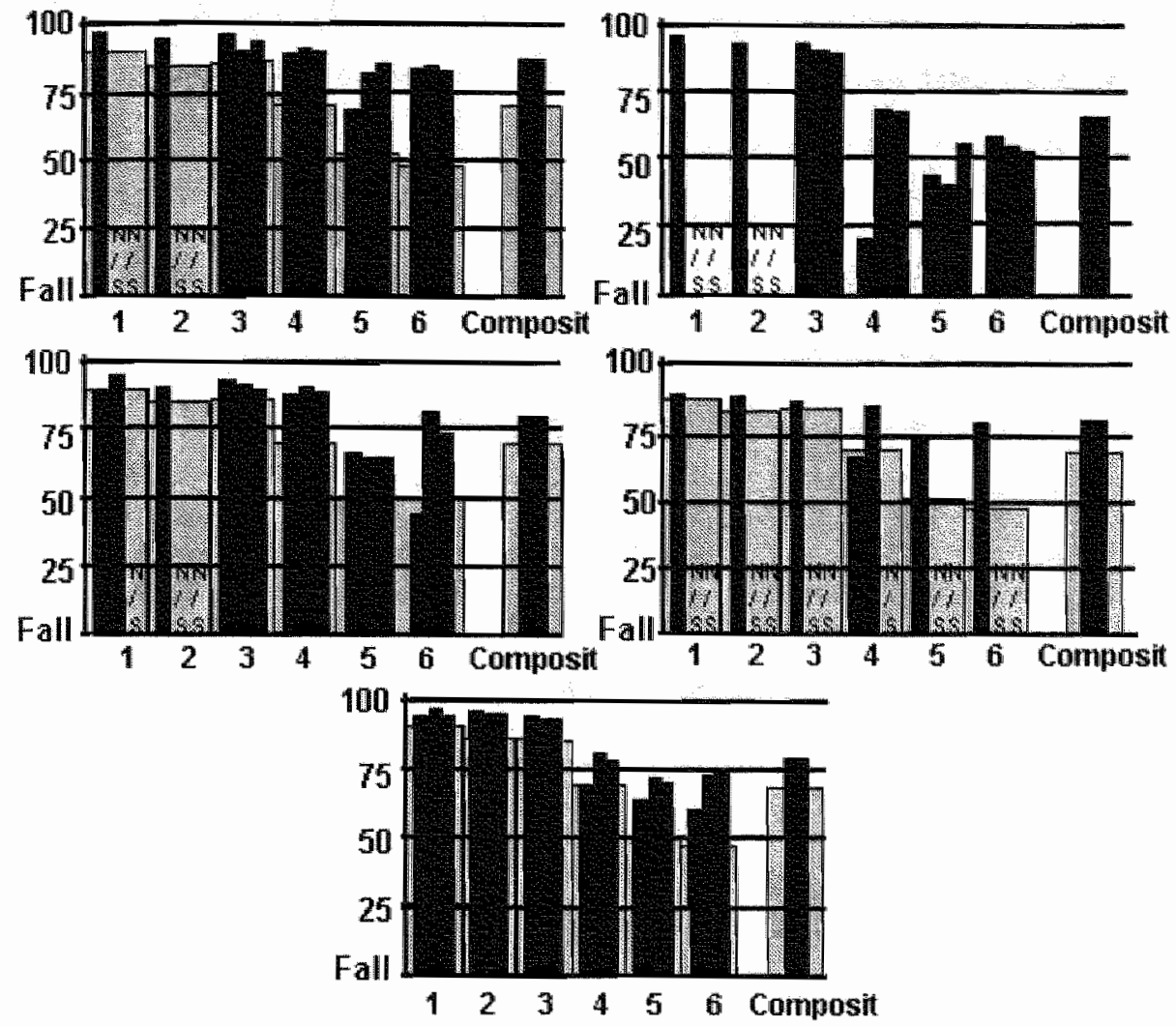

Figure 5. The equilibrium score of the sensory organisation test of five healthy individuals. The equilibrium score is approximately the same or even better in condition 6 than in 5 .

N/S: no score (test not repeated).

Therefore, no firm or large base of support is required. Also, the hip strategy allows a rapid movement of the $\mathrm{COG}$ when the $\mathrm{COG}$ is positioned near the limit of stability and therefore it is the strategy of choice to prevent a possible fall (35). In case of the hip strategy, the translational force exerted on the lower trunk in the horizontal plane is partly counteracted by an opposite translational (shear) force exerted by the feet in the plane of the supporting surface. The patient's body movement strategies can be detected through a shear force transducer mounted in the platform. The transducer measures the horizontal shear forces exerted on the platform whenever the body's segments accelerates. For the ankle movement strategy, the COG accelerations and shear forces are small with low frequencies (below $0.5 \mathrm{~Hz}$ ). For the hip movement 
strategy, the body segment accelerations and shear forces are large with frequencies above $1 \mathrm{~Hz}(34)$.

Strategy scores are calculated by comparing the peak-to-peak amplitude of the shear force oscillation to the maximum possible shear force. Figure $6 \mathrm{~b}$ indicates that the theoretical maximum possible shear force (B) a subject can induce is estimated to be $11.4 \mathrm{~kg}$ (23). A strategy score of $100 \%$ points to a pure ankle strategy (figure $6 \mathrm{a}$, no shear force), whereas a score of $0 \%$ indicates a pure hip strategy (figure $6 \mathrm{a}$, maximum shear force). The strategy score is presented in a bar graph (figure 6c).

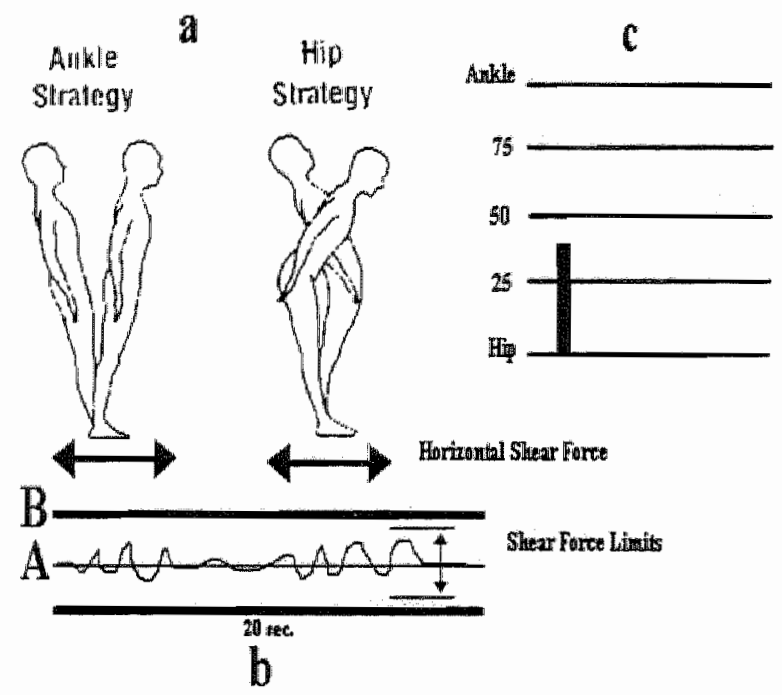

Figure 6 .

Body movement strategy score.

A. actual shear force exerted in the plane of the platform.

B. theoretical maximum shear force (21).

a. visualisation of the ankle and hip strategy.

b. graphical representation of the shear force exerted in the place of the piatform (A) and the theoretical maximum shear force.

c. relative score of ankle versus hip strategy component $(100 \%=$ pure ankle strategy, $0 \%=$ pure hip strategy).

There are a number of reported abnormal body movement strategy's scores provided during the SOT which have been attributed to different vestibular disorders $(37,38$, 39). The theory of body movement strategy is based on the assumption that body sway is confined to the anterior-posterior plane and is limited to the ankle and hip joints (23), which however needs more verification. At last, hip and ankle movement strategy is not detected as accurately by the force platforms as suggested $(36,40)$. This suggests that other methods of body movement detection may be preferable. 
To summarise, dynamic posturography still seems to be a complementary examination, adding specific information to the classical vestibular tests that evaluate the vestibuloocular reflexes. However, even the current type of dynamic posturography broadly applied has to be considered as a functional test of balance with limited dimgnostic capabilities (22). An even more direct point of interest is to which extent vestibular deficiencies cause disturbed posture through the vestibulo-spinal reflex (8). The question also arises whether it will be possible to modify the measurement procedure and conditions such that more discriminative and reproducible response pattems can be obtained.

The objective of this thesis is:

To develop a new concept of the dynamic posturography to improve reproducibility and specificity (chapter 2 and 3 ).

To evaluate and compare the different methods used in literature to disturb the proprioceptive and visual system (chapter 3 and 5).

To assess the efficacy of dynamic posturography in measuring the balance training outcome in healthy subjects (chapter 4).

To investigate the head and body movement strategies used to maintain balance control (chapter 6). 


\section{References}

1. Terekhov Y. Stabilometry and some aspects of its applications-a review (1976). Biomed. Eng. 11:12-15.

2. Mirka A, Black FO. Clinical application of dynamic posturography for evaluating sensory integration and vestibular dysfunction (1990). Neurol. Clin. 8:351-359.

3. Shepard NT. The clinical use of dynamic posturography in the elderly (1989). Ear Nose Throat J. 68:940-957.

4. Hamid MA. Chronic dizziness: vestibular evaluation and rehabilitation (1994). Clevel. Clin. J. Med. 61:247-249.

5. Granit R, Pompeiano, O (eds.). Prog. Brain Res. 50 (1979). Amsterdam: Elsevier Scientific Publishers 827.

6. Pompeiano O, Allum JHJ. Vestibulo-spinal control of posture and locomotion (1988). Prog. Brain Res. Amsterdam: Elsevier Scientific Publishers.

7. Norré ME, Forrez G. Vestibulospinal function in otoneurology (1986). ORL. J. Otorhinolaryngol. Relat. Spec. 48:37-44.

8. Norré ME, Forrez G. Posture testing (posturography) in the diagnosis of peripheral vestibular pathology (1986). Arch. Otorhinolaryngol. 243:186-189.

9. Romberg MH. Manual of nervous diseases of man (1853). London: Sidenham Society, 935-941.

10. Black FO. Vestibulospinal function assessment by moving platform posturography (1985). Am. J. Otol. 39-46.

11. Norré ME. Posture in otoneurology (1990). Acta Oto-Rhino-Laryngol. Belg. 44:355-364.

12. Norre ME. Contribution of a posturographic six-test set to the evaluation of patients with peripheral vestibular disorders (1992). J. Vestibular Res. 2:159166.

13. Roberts TDM. Neurophysiology of postural mechanisms (1978). $2^{\text {nd }}$ edition. London: Butterworths \& Co.

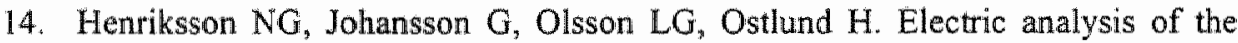
Romberg test (1966). Acta Oto-Laryngol. Suppl. 224:272-279.

15. Taguchi $\mathrm{K}$. Spectral analysis of body sway (1977). ORL. J. Otorhinolaryngol. Relat. Spec. 39:330-337.

16. Shumway-Cook A. Horak FB. Assessing the influence of sensory interaction on balance: suggestions from the field (1986). Phys. Ther. 66:1548-1550.

17. Bles W. Sensory interactions and human posture (1979). Thesis, Free University, Amsterdam.

18. Diener HC, Dichgans J, Bruzek W, Selinka H. Stabilization of human posture during induced oscillations of the body (1982). Exp. Brain Res. 45:126-132.

19. Black FO, Nashner LM. Vestibulospinal control differs in patients with reduced versus distorted vestibular function (1984). Acta Oto-Laryngol. Suppl. 406:110114.

20. Black FO, Wall C, Rockette HE, Kitch R. Normal subject postural sway during the Romberg test (1982). Am. J. Otolaryngol. 3:309-318. 
21. Neurocom Int. Corp. Equi Test Data Interpretation Manual (1991). Clackamas, Ore..

22. Nashner LM, Peters JF. Dynamic posturography in the diagnosis and management of dizziness and balance disorders (1990). Neurol. Clin. 8:331-349.

23. Nashner LM, Shupert CL, Horak FB, Black FO. Organization of posture controls: an analysis of sensory and mechanical constraints (1989). Prog. Brain Res. $80: 411-418$.

24. Horak FB. Clinical measurement of postural control in adults (1987). Phys. Ther. $67: 1881-1885$.

25. Tangeman $P$, Wheeler J. Inner ear concussion syndrome: vestibular implications and physical therapy (1986). Top Acute Care Trauma Rehab. 1:72-83.

26. Black FO. Vestibular function assessment in patients with Meniere's disease: the vestibulo-spinal system (1982). Laryngoscope 92:1419-1436.

27. Black FO, Nashner LM. Postural control in four classes of vestibular abnormalities (1985). In: Igarashi M, Black $\mathbb{F O}$ (eds.), Vestibular and Visual control on Posture and Locomotor Equilibrium. New York: Karger Publications, 271-281.

28. Hamid MA, Hughes GB, Kinney SE. Specificity and sensitivity of dynamic posturography. A retrospective analysis (1991). Acta Oto-Laryngol. Suppl. 481:596-600.

29. Voorhees RL. Dynamic posturography findings in central nervous system disorders (1990). Otolaryngol. Head Neck Surg. 103:96-101.

30. Furman JM. Role of posturography in the management of vestibular patients (1995). Otolaryngol. Head Neck Surg. 112:8-15.

31. Barin K, Seitz, CM, Welling, DB. Effect of head orientation on the diagnostic sensitivity of posturography in patients with compensated unilateral lesions (1992). Otolaryngol. Head Neck Surg. 106:355-362.

32. Nashner LM. Organization of human postural movements during standing and walking (1986). In: Grillner S, Stein PSG, Stewart DG, Forssberg H, Herman $\mathbb{R}$. (eds.), Neurobiology of posture and locomotion. London: MacMillan Pres\$ 637. 648.

33. Nashner Li, McCollum G. The organization of human postural movements: a formal basis and experimental synthesis (1985). Behav. Brain Sci. 8:135-172.

34. Horak FB, Nashner LM. Central programming of postural movements: adaptation to altered support surface configurations (1986). J. Neurophysiol. 55:1369-1381.

35. Moore SP, Horak FB, Nashner LM. Influence of initial stance position on human postural response (1986). Soc. Neuros. 12:1301.

36. Berendsen T. Quantitative mechanical analysis of the detection of position of body COG by a force platform (1991). Thesis, Technical University Twente.

37. Black FO, Shupert CL. Horak FB. Abnormal postural control associated with peripheral vestibular disorders (1988). Prog. Brain Res. 76:263-275.

38. Shupert CL, Nashner LM, Horak FB, Black FO. Coordination of the head and body in standing posture in normals and patients with bilaterally reduced vestibular function (1987). Soc. Neuros. 13:352. 
39. Shupert CL, Horak FB, Black FO. Abnormal postural coordination in patients with distorted vestibular function (1988). Soc. Neuros. 14:65.

40. Hamman RG, Mekjavic I, Mallinson AI, Longridge NS. Training effects during repeated therapy sessions of balance training using visual feedback (1992). Arch. Phys. Med. Rehabill 73:738-744.

41. Diener HC, Dichgans J. Anwendung und nutzen der statischen und dynamische standmessung (Posturograpie) (1988). Forschr. Neurol. Psychiatr. 56: 249-258.

42. Allum $\sqrt{H}$, Honegger $F_{*}$ Schicks $H$. The influence of a bilateral peripheral vestibular deficit on postural synthesis (1994). J. Vestibular Res. 4: 49-70. 
Chapter 2

\section{Dynamic posturography in healthy subjects: evaluation of COG measuring techniques and individualisation of test conditions}




\section{Abstract}

In this study two problems are addressed and evaluated by studying balance control in 11 healthy subjects.

\section{Measurement technique}

The first problem concerns the fundamental limitation of the force platform as a measuring device applied in posturography. An altemative technique based upon infrared (IR)-video tracking of five markers fixed to the head, shoulder, hip, knee and ankle was developed to calculate the position of the body centre of gravity $(\mathrm{COG})$; this technique is compared with techniques using a force platform or a single body fixed angular rate sensor. The fundamental question is put forward whether force transducers mounted in platforms are adequate sensors to detect $\mathrm{COG}$ position specially when applied in dynamic conditions (vigorous moving subjects or platforms).

\section{Quantification of balance control near the limit of stability}

The second problem concerns the poor reproducibility and low specificity of dynamic posturography: test performance is related to the functional status of the subjects balance system, and the subject's motivation and interpretation of the test-instruction. "Good" patients appear to perform better than "bad" normal subjects and most subjects show a steady improvement during successive testing (habituation and adaptation). To cope with this problem a technique is introduced to quantify balance control near the limit of stability to force the subject to an optimal performance. We hypothesise that in this way every subject is tested according to the individual abilities and that reproducibility might improve when compared to more static, non individualised or less challenging test conditions.

Ad. I. Measurement technique. The output of the three detection systems and a video recording of the actual body movements were compared in six different body and/or platform movement conditions. The force transducers detect the centre of pressure of the feet, which induces considerable systematic errors in the calculation of the actual body sway at physiological movement frequencies above $0.2 \mathrm{~Hz}$. We conclude that the value of dynamic posturography using force platforms should be considered in line with the limited accuracy of this method to detect the COG position under dynamic conditions. Application of one single angular rate sensor neither provides an accurate detection of the COG position nor velocity. In contrast, despite some theoretical limitations related to the biomechanical models to be used, we conclude that the video tracking method produced the most accurate and reliable estimate of the COG movement, specially in case of substantial body movements at frequencies above $0.2 \mathrm{~Hz}$.

Ad. II. Quantification of balance control near the limit of stability. 11 healthy subjects stood with eyes open on a motor driven platform that rotated around the ankle axis pseudo-randomly. COG position and velocity were real time monitored by means of the video system. During each test the amplitude of the platform movement was maximised automatically as long as the body movement was in accordance with pre-set COG velocity and COG position limits. Four physically active subjects tolerated higher platform amplitudes than three moderately active subjects; the 
remaining four relatively inactive subjects tolerated only small platform movements. The test score thus correlated well with the subjects own judgement of balance capabilities in daily life in contrast with the findings of the routine stabilometry that showed normal and not-significantly different balance control in all 11 subjects. Reproducibility of the test scores was within $14.5 \%$.

\section{Introduction}

Upright stance has an enormous significance for the human being, because it frees the hands for productive activity (1) and improves visual orientation. Romberg was among the first to recognise the complex sensorimotor interactions required for control of upright stance (2). In his classical test the patient suffering from a poor balance is asked to stand erect, eyes open, the feet slightly separated from each other and balance control is judged. Romberg originally devised this test to demonstrate postural instability in case of deficits of the posterior column, but was fully aware of the influence of input from vision, labyrinths and general propriocepsis upon balance control.

Postural sway is considered to reflect the physiological postural muscular activity necessary to keep in balance. This muscular activity induces a continuous to-and-fro movement of the centre of gravity of the body (COG) around the required position $(3,4,5)$. A 'small' body sway is therefore associated with a normal function of the balance control system, whereas a 'large' body sway or fall is considered characteristic for deficits anywhere in the balance control. system. However the large inter- and intra-individual variability of the test, as well as the large inter-observer variability, resulted in a low appreciation of the test as a part of the vestibular test battery. Therefore considerable improvement was expected from the introduction of stabilometry which can be considered as the quantitative version of Romberg's test.

\section{Stabilometry: quantification of balance control}

Stabilometry became available through the development of force platforms that enabled detection and quantification of lateral and anterior-posterior body sway (6). Here the patient stands on a platform (often called force plate) that is equipped with minimal three force transducers. The transducers detect the vertical forces exerted by the feet on the platform and inform about the force distribution present over the platform. The force distribution changes in case of displacements of the COG. As a consequence detection of the force distribution is used to calculate the absolute position of the vertical projection of the COG on the platform. In this way body sway direction, amplitude and velocity are generally quantified $(1,7)$. Stabilometry (or static posturography) has long been proposed as a relevant tool to examine patients with instability $(3,5,8,9,10)$. However, despite many modifications and refinements of the test, the poor reproducibility and low specificity appeared to be consistent and prevented a break through of stabilometry as a relevant diagnostic tool. Stabilometry has not been shown to yield information beyond that obtained from other diagnostic procedures (10).

\section{Dynamic posturography}

In a second phase of innovative experiments, moving platforms were used to study balance control. This type of posturography is better known as dynamic posturography. The technique 
was introduced to create a stimulus-response condition or to manipulate the proprioceptive input to the balance control system. Primary, fast moving platforms were used as stimulators to induce postural reflexes, detect movements of body segments and EMG of postural muscles $(11,12)$. This application proved to be useful in diagnostics of neuromuscular diseases and to a limited extent to the diagnostics of diseases of the balance system. Secondary, another class of dynamic posturography was introduced: sway referenced platforms were applied to reduce or mislead the proprioceptive input in a sophisticated and computer controlled way in combination with a sway referenced visual surround (13). In this technique the anteriorposterior movements of the COG of the subject are detected by the platform and fed into a computer that controls the rotation angle of a tilting platform and a visual surround. Both platform and visual surround, either simultaneously or separately, rotate around an axis through the ankle joints with an angle proportional to the anterior-posterior body sway angle. In this way the orientation of the surface and/or visual surround relative to the body remains constant despite the anterior-posterior body movements suggesting that the subjects spatial orientation to the gravitational vector does not change, whereas the opposite is true.

\section{Basic problems}

First, a fundamental question arises about the measuring techniques applied in almost all posturography platforms $(13,14)$. Under strictly static conditions the point of maximum pressure on the platform coincides with the position of the vertical projection of the COG on the platform. In this way, body movements expressed in terms of movements of the COG are generally used for quantification of posture. However changes in the distribution of foot pressure on the platform during stance, or vigorous body or platform movements will complicate the detection of body movements through this method. Specially, during active movement of the platform reaction forces do occur and interfere with the detection of the COG. These phenomena obstruct an accurate detection of body sway $(14,15)$.

Second, despite many modifications and refinements both stabilometry and dynamic posturography still suffer from a poor reproducibility and low specificity (16), partly because of the limited accuracy of the measuring techniques, but also because the test performance is related to the functional status of the subjects balance system, and the subjects motivation and interpretation of the test-instruction. In addition, "good" patients (a ballet dancer after a unilateral labyrinthine deficit) appear to perform better than "bad" normal subjects (a professional bridge player that hates to move) and most subjects show a steady improvement during successive testing (habituation and adaptation).

\section{Aim of the study}

In this study both problems are addressed. First an alternative measurement technique of anterior-posterior body sway (frequently used in gait analysis) based upon infrared (IR)-video tracking was developed and compared with techniques using force transducers or accelerometers. Second, a technique is introduced which is hypothesised to allow quantification of balance control near the limit of stability: every subject is tested according to the individual abilities in order to improve reproducibility. 


\section{Subjects}

After informed consent 11 healthy subjects ( 5 females, 6 males, $23-32$ years old) were included in this study. The subjects had no history of neurologic or musculoskeletal problems that would affect their balance and orientation. They had no history of episodes of dizziness nor underwent any ear operation and were not taking any medication at the time of testing. All subjects showed a normal oto-neurological status and audiometry and standard vestibular testing (spontaneous, gaze, saccades, optokinetics, smooth pursuit, torsion swing, calorics) revealed no abnormalities. Four subjects were active in sports (basketball, running, ballet), three had a moderately active life (no substantial regular sport activities) and four subjects considered themselves as non-sportive and relatively inactive. Body sway velocity, body sway area, as well as the associated Romberg coefficients of all subjects, as revealed by computerised stabilometry, were well within the normal limits. No significant difference could be observed between the three groups (active, moderately active, inactive).

\section{Methods}

The experimental set-up was composed of two IBM compatible 486-DX-66 PC's (master and slave), an infrared (IR)-video tracking system, an angular rate sensor (ARS-C131-1A, Watsons Industries, Eau Claire, USA) a dynamic platform equipped with four force transducers and a potentiometer that detected the platform position (T-Post, Jaeger-Toennies $\mathrm{GmbH}$, Wuerzburg, Germany). The master computer was equipped with an indlexer card (PC-21 Parker Hannifin Corporation, Petaluma, $\mathrm{Ca}$, USA) to control the dynamic platform and hosted an AD converter (Bur Brown 200898 channel, 12 bits, ) for data-acquisition and an ether-net card for datatransfer to the departmental local-area network. Special software (Borland $\mathrm{C} / \mathrm{C}++$ ) was developed for data-acquisition, analysis and stimulus control (17). Besides the event and time control of the test procedures, the software was able to use any input signal to control any output signal by a user defined transfer function. The slave computer hosted a framegrabber (VFG Visionetics, $512 \times 512,8$ bits) together with an IR-video camera (HTC Eindhoven, The Netherlands, Sony MZ CCD 1/3" BW) and served as a tracking system for body moveninents. Special software was developed for real-time tracking of active IR-markers attached to the body of the subject under investigation. The software was a modification of the software developed for the binocular real-time 3D IR-video eye-tracker used for eye-position detection (18).

\section{Detection and quantification of body movements in the anterior-posterior plane}

The first method to be described here is the frequently used method of quantification of body movements by using a platform provided with force transducers (strain gauges) or pressure sensors (piezo-electric sensors). We used a commercially available platform with calibrated force transducers mounted in each comer of the rectangular platform. The principle of detection of body sway is as follows. During the measurements the subject stands in the middle on the platform, the feet positioned between the four transducers. The anterior-posterior position of the point of maximal pressure is detected by comparing the output of the two force transducers positioned in front of the subject to the output of the force transducers positioned behind the 
subject. The lateral position of the point of maximum pressure is obtained by comparing the output of the two transducers positioned on the right side of the subject with those positioned at the left side $(19,20,21,22)$.

The second method applied made use of an angular rate sensor which was mounted on the back of the subject close to the position of the COG which lies at the level of the sacrum (about $56 \%$ of the length of the human body, $(19,20,21)$. The anterior-posterior COG velocity $(\mathrm{cm} . / \mathrm{sec}$.) could be directly calculated from the angular rate sensor output with use of this estimate of the vertical position of the $C O G$. The anterior-posterior $C O G$ position was derived from the $C O G$ velocity by digital integration (time constant $100 \mathrm{msec}$.). It must be stressed, however, that the use of a velocity sensor does not allow an accurate detection of the absolute position of the COG due to the small but relevant off set and drift of the detector output.

The third method used to detect body movements was established by a video imaging technique. Five active IR-lights ( $>900 \mathrm{~nm}$ ) were mounted in such a way on the ankle joint, knee joint, hip joint, shoulder and head, that a camera positioned lateral of the subject could detect all markers. The video-image was fed through a frame grabber into the slave-PC. In each frame, the $\mathrm{X}-\mathrm{Y}$ co-ordinates of each marker were detected real time by special software developed at the department of Otorhinolaryngology (18) and subsequently transferred through the serial interface to the master PC. Based upon the position of all five IR markers, using a simple biomechanical model (23), the position of the COG in the anterior-posterior plane could be detected real time (figure 1). The computer calculated the position of the COG. The delay between actual body movement and the calculation of the associated movement (position) of the COG, was always less than $13 \mathrm{msec}$. Body sway velocity was calculated by differentiating ( 3 points) the digitised position signal. The parameters used in the model are as follows (23):

body segments

mass $(\%)$
A (Head).
B (Trunk + Arms).
$C$ (Upper Legs).
D (Lawer Legs + Feet).

$$
\begin{aligned}
& \delta \mathrm{A}=0.00 \\
& \delta \mathrm{B}=51.2 \\
& \delta \mathrm{C}=43.3 \\
& \delta \mathrm{D}=58.5
\end{aligned}
$$

body segments

\section{distance of body segment COG \\ to the marker, top-down (\%)}

$$
\begin{aligned}
& \mu A=8.5 \\
& \mu B=57.9 \\
& \mu C=21.3 \\
& \mu D=12.3
\end{aligned}
$$

The $x-y$ position of the COG of each body segment was defined relative to the initial position $(\mathrm{X} O, \mathrm{Y} 0)$ by:

$$
\begin{array}{ll}
X \operatorname{cog} A=(1-\delta A) * X 0+\delta A * X 1 & Y \operatorname{cog} A=(1-\delta A) * Y 0+\delta A * Y 1 \\
X \operatorname{cog} B=(1-\delta B) * X 2+\delta B * X 2 & Y \operatorname{cog} B=(1-\delta B) Y^{*} 1+\delta B * Y 2 \\
X \operatorname{cog} C=(1-\delta C) * X 2+\delta C * X 3 & Y \operatorname{cog} C=(1-\delta C) * Y 2+\delta C * Y 3 \\
X \operatorname{cog} D=(1-\delta D) * X 3+\delta D * X 4 & Y \operatorname{cog} D=(1-\delta D) * Y 3+\delta D * Y 4
\end{array}
$$

Which allows calculation of the $x-y$ position of the whole body $C O G$ :

$$
\begin{aligned}
& \mathrm{X} \operatorname{cog}=\Sigma(\mathrm{Xi} * \mathrm{Mi})=\Sigma(\mathrm{Xi} * \mu \mathrm{i})=\mathrm{XA} * \mu \mathrm{A}+\mathrm{XB} * \mu \mathrm{B}+\mathrm{XC} * \mu \mathrm{C}+\mathrm{XD} * \mu \mathrm{D} \\
& \mathrm{M} \\
& \mathrm{Ycog}=\Sigma\left(\mathrm{Yi}^{*} \mathrm{Mi}\right)=\Sigma\left(\mathrm{Yi}^{*} \mu \mathrm{i}\right)=\mathrm{YA} * \mu \mathrm{A}+\mathrm{YB} * \mu \mathrm{B}+\mathrm{YC} * \mu \mathrm{C}+\mathrm{YD} * \mu \mathrm{D} \\
& \mathrm{M}
\end{aligned}
$$




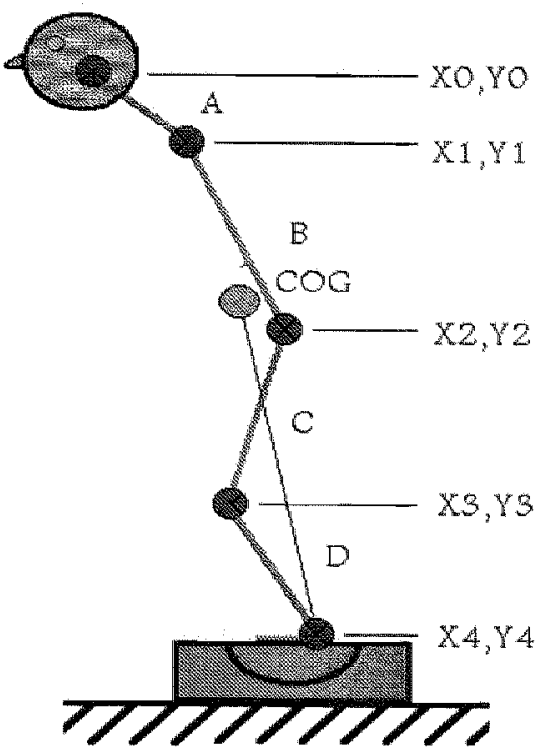

Figure 1. Schematic drawing of the biomechanical model described by Winter (23). The symbols are explained in the text.

General aspects of stimulation and signal processing

The subject stood bare feet on the dynamic platform. All measurements were performed with eyes open. Temporal vision was blocked by special goggles. A slide projector imaged a (slightly out of focus) random dot pattern on a transview screen (width $3.20 \mathrm{~m}$., height 2 . $40 \mathrm{~m}$.) $0.5 \mathrm{~m}$ meter in front of the subject to allow normal visual orientation. The subjects were instructed to stand as stable as possible without leaning forward or backward during the test period, with the hands folded at the waist, resting on the frontal part of the body. The dynamic platform could rotate maximum $9 \mathrm{dg}$ forwards and $9 \mathrm{dg}$ backwards with a maximum angular acceleration of $1400 \mathrm{dg} / \mathrm{sec}^{2}$. The axis of rotation was oriented horizontally through the ankle joints. Body movements were detected (separately or simultaneously) by the four force transducers mounted in the platform at each corner (detection of the centre of pressure), by a calibrated angular rate sensor mounted on the back at the level of the sacrum and by the videotracking system. The analogue output of the four force transducers and the angular rate sensor, together with the platform angle (generated by a potentiometer mounted on the platform) were fed into the $\mathrm{AD}$-converter of the master computer and sampled at $50 \mathrm{~Hz}$. The $\mathrm{x}-\mathrm{y}$ position of the five IR-markers detected by the video-tracker were fed into the master PC through the RS232 serial interface.

The $x-y$ position of the centre of pressure (derived from the force transducers), body velocity (angular rate sensor), horizontal and vertical position of the IR-markers (attached to the ankle 
joint, knee, hip, shoulder and head), the platform angle were all stored in a datafile in the computer when required. When reading these stored data, body movements could be replayed on the computer screen (schematic drawing of the subject) as a function of time and correlated to all other stored parameters. Any parameter stored could be displayed as a function of time.

\section{Experiments}

\section{Experiment 1. evaluation of measuring techniques}

Anterior-posterior body movements were detected by the three different techniques simultaneously: a force platform, an angular rate sensor mounted on the subjects back, and by IR video imaging of five body fixed $\mathbb{R}$-markers. All body movements were video taped and used to compare the actual body movements with the output of these three detection systems, to allow a clear interpretation. Six experiments $(1 \mathrm{a}-1 \mathrm{f})$ were performed.

Experiment la. The subjects were asked to exert a force with the toes upon the force plate without moving the body (change of force distribution without COG displacement).

Experiment $I b$. The subjects were asked to move the head for and backwards (slow head nodding), while standing as stable as possible on a stationary platform (COG displacement without active change of foot pressure distribution).

Experiment 1c. The subjects were asked, standing on a stable platform, to move the upper trunk slowly maximally forwards with simultaneous slow movement of the lower trunk in the opposite direction and vice versa (change of body segment orientation with minimal a COG displacement).

Experiment Id. The subjects were standing as stable as possible, using pure ankle rotation to cope with a predictable $0.57 \mathrm{~Hz}$ sinusoidal rotation of the platform (no change of force distribution, no COG displacement, no body segment displacement apart from ankle rotation). Experiment $l e$. The subjects were asked to move the body very slowly for and backward ("pseudo-static" condition), while standing on a stable platform (passive change of force distribution related to $\mathrm{COG}$ displacement, body segment displacement).

Experiment lf. The subjects were asked to move their body vigorously ("dynamic" condition), while standing on a stable platform (active and passive change of force distribution, $\mathrm{COG}$ displacement, body segments displacements).

Experiment 2. development of a method to quantify balance control at the limit of stability $C O G$ position and velocity were continuously measured by means of the IR-video system. The platform movement consisted out of 3 mutual harmonically unrelated frequencies $(0.16,0.42$, $1.15 \mathrm{~Hz}$ ) with an initial sum of all three amplitudes of $2 \mathrm{dg}$. During the duration of the experiment the amplitude of the platform movement (APM) increased automatically in time with $0.004 \mathrm{dg}$ per $20 \mathrm{msec}$. $(0.2 \mathrm{dg} / \mathrm{sec}$.) as long as COG velocity was below $5 \mathrm{~cm} / \mathrm{sec}$. APM was fixed when the subject could maintain the COG velocity within the range from $5-8$ $\mathrm{cm} / \mathrm{sec}$. We observed that the body sway velocity tends to decrease when the subjects leaned more than about 3 degrees forward or backward. We therefore concluded that body sway velocity alone is not a sufficient indication for the postural system capabilities, but that body position relative to the absolute vertical is also essential in the evaluation of balance control, and should be used as a criterium as well. Therefore, APM automatically decreased with 0.004 dg per $20 \mathrm{msec}$. $(0.2 \mathrm{dg} / \mathrm{sec}$ ) when COG velocity exceeded $8 \mathrm{~cm} / \mathrm{sec}$. APM also decreased 
whenever $50 \%$ ( $3.125 \mathrm{dg}$ ) of the maximum angle (6.25 dg) of forward or backward leaning ("the cone of stability") was reached irrespective the actual body sway velocity, to prevent the subject from falling. These position $(3.125 \mathrm{dg})$ and velocity criteria $(5-8 \mathrm{~cm} / \mathrm{sec}$.) were determined in a pillot study in 7 other healthy subjects.

In this pilot study it was observed that, during quiet stance and normal vision, all subjects showed COG peak velocities well below $3 \mathrm{~cm} / \mathrm{sec}$. Upon sudden unexpected toe-up rotations of the platform ( $9 \mathrm{dg}$ step, $1400 \mathrm{dg} / \mathrm{sec}^{2}$ acceleration) 5 subjects tended to fall or performed gross body movements to regain balance with $C O G$ peak velocities exceeding $9 \mathrm{~cm} / \mathrm{sec}$. and COG positions orientated more than $5 \mathrm{dg}$ forward or $4 \mathrm{dg}$ backward. In the same pilot study test duration varied from 20 to maximum 60 seconds. APM first inclined during the first $20-30$ seconds, but subsequently showed an irregular and not reproducible pattern in most subjects when the test duration exceeded 30 seconds. Most subjects reported with this respect that it was rather difficult to persevere optimal performance for more than 20 seconds, without large instabilities or big corrective body movements. All subsequent tests were therefore performed with a maximum duration of 20 seconds. Balance control was expressed in terms of the Tolerated Platform Movement 'TPM' quantified as the average APM tolerated during the 20 seconds test duration. With an initial APM of $2 \mathrm{dg}$ and a maximum increase of $0.2 \mathrm{dg} / \mathrm{sec}$. this resulted in a maximum APM of $6 \mathrm{dg}$ after 20 seconds and a maximum TPM of $4 \mathrm{dg}$. All subjects were tested three times ( 20 seconds each) with a time interval of 10 minutes between each test, to evaluate the reproducibility of the method and habituation to the stimulus and measuring conditions.

\section{Data analysis}

Statistical analysis was performed using PC-SPSS version 7.5.2.

\section{Results}

\section{Experiment la-lf. Evaluation of measuring techniques}

The output of the angular rate sensor was generally more hampered by noise than the wideo signal or the output of the force transducers. Also, this sensor suffers from a small off-set and drift, a problem which is accumulated in case of integration of the signal in time to allow a detection of $C O G$ position: the $C O G$ position error could mount more than $10 \mathrm{~cm}$ within 60 seconds. Additionally, it was found to be hard to fix the rate sensor firm enough to prevent motion relative to the body, especially at higher body velocities.

Figures $2 \mathrm{~A}$ to $2 \mathrm{~F}$ show the results in a subject that underwent experiment la through $1 \mathrm{f}$. Similar results were obtained in all other 10 subjects.

\section{Experiment 2. Development of a method to quantify balance control at the limit of stability}

Figure 3 shows the data screen after the 20 seconds measurement of balance control in a healthy subject during continuous platform movement. Within all subjects the averaged ( $n=$ 11) tolerated TPM ranged from 2.9 to $3.9 \mathrm{dg}$, with a mean value of $3.5 \mathrm{dg}$. Test-retest variation range was always less than $0.48(<14.5 \%)$ substantially smaller than the inter-individual range 
of $0.9 \mathrm{dg}$. The active group tolerated higher APM's: all showed an almost continuous increase of APM and scored near the maximum TPM of $4.0 \mathrm{dg}(3.7,3.8,3.9,3.9)$ and judged the test as "easy". Tenfold repetition of this test within a time span of maximum $30 \mathrm{~min}$. showed no significant change ( $>>0.05$, Wilcoxon Matched Pairs Signed Rank test). The moderately active group scored TPM"s of $3.4,3.6$ and $3.8 \mathrm{dg}$ and judged the test as "challenging, but not difficult"; they generally showed a steady increase of APM, only incidentally interrupted by a short duration plateau. Repetition of the test (tenfold) directly after the first session in the moderately active subjects showed that they steadily improved their TPM score up to 3.6 to $3.9 \mathrm{dg}$. The inactive group tolerated relatively low A.PM's (TPM: $2.9,3.0,3.2$ and $3.3 \mathrm{dg}$ ); all four subjects showed a steady increase of APM interrupted by several plateau or even short duration decreases and reached a final plateau near the end of the test. Repetition of the experiments in this group did not show a consistent improvement of TPM score. In contrast to the more active subjects these subjects reported that they experienced the test as "rather difficult" and that they were afraid to fall.

\section{Interpretation and Discussion}

\section{Evaluation of the three measuring techniques}

The problems with a firm fixation to the body, the poor signal to noise ratio and the large position error made us conclude that the detection technique using the angular rate sensor is less accurate when compared to the other two techniques applied in this study. However, a more decisive conclusion could be made based upon the results of experiment 1 . When subjects were asked to exert an active force on the platform by the toes, avoiding substantial body movements (experiment $1 \mathrm{a}$ ) the video tracking system and the angular rate sensor did not sense substantial body movement, whereas the force transducers correctly indicated a change of force distribution under the feet. The latter could wrongly be interpreted as a displacement of the COG (figure $2 a$, upper trace). When the subjects were asked to move the head, while standing as stable as possible on a stationary platform (experiment 1.b), the position of the COG calculated by the force transducers and the video-tracking system moved accordingly. However, the body fixed angular rate sensor was unable to detect the substantial COG movement (figure $2 \mathrm{~b}$ ). When the subjects were asked, standing on a stable platform, to move the upper trunk forwards with simultaneous movement of the lower trunk in the opposite direction (experiment lo), the position of the COG detected by the force transducers and the video-tracking system hardly moved. In contrast, the angular rate sensor did detect wrongly a substantial COG movement (figure2c). When the subjects were standing as stable as possible, using pure ankle rotation to cope with a predictable sinusoidal rotation of the platform (experiment $1 \mathrm{~d}$ ), neither the angular rate sensor, nor the video-tracking system indicated any substantial movement of the $\mathrm{COG}$; only the sensors in the force platform detected a small sinusoidal movement. When the subjects were asked to move the body very slowly for and backward (experiment $\mathbb{l}$ e, figure 2e) all three detection systems signalled the COG displacement similarly and correctly. 
a

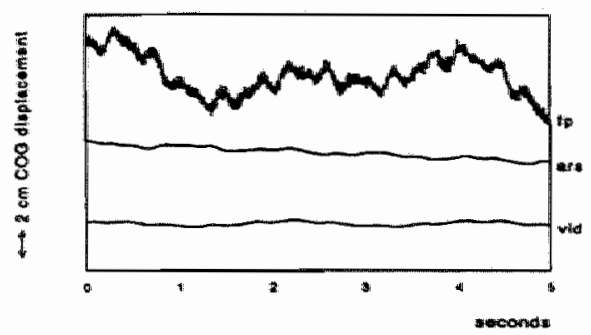

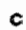

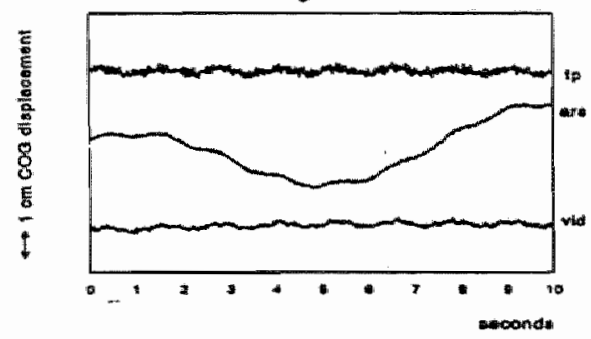

e

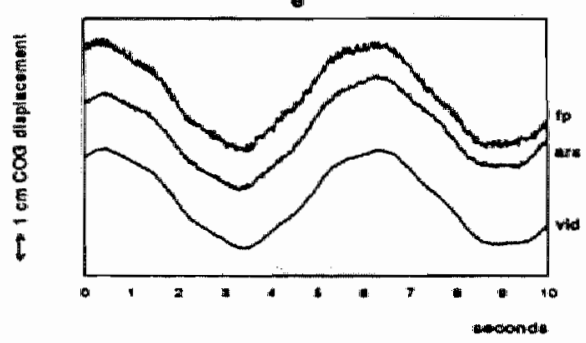

b

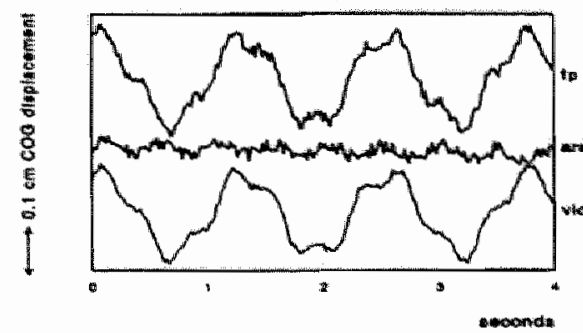

di

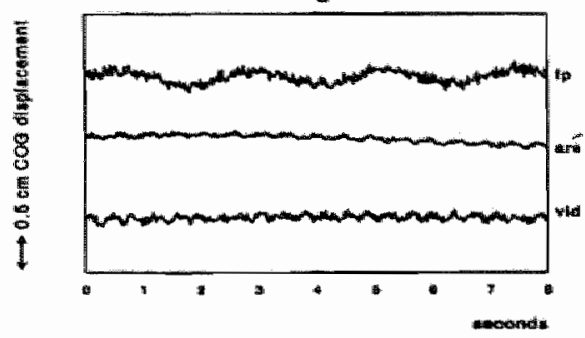

1

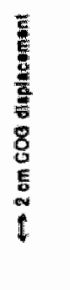

Figure 2 a-f. Displacement of COG as a function of time in one healthy subject detected by a force platform (upper traces, fp), an angular rate sensor mounted on the subjects back (middle traces, ars), and by IR-video imaging of five body fixed IR-markers (lower traces, vid). Mind different scales of $y$ and $x$-axes. Figure 2a: stable platform, toes exert a force upon the force plate without body movement. Figure 2b: stable platform, slow head nodding. Figure 2c: stable platform, simultaneous slow movement of upper trunk maximally forwards and lower trunk backward, vice versa. Figure $2 d$ : predictable moderately slow $0.57 \mathrm{~Hz}$ sinusoidal platform rotation, 'pure' ankle rotation. Figure 2e: stable platform, very slow body movements for and backwards. Figure 2f: stable platform, vigorous body movements for and backwards. 


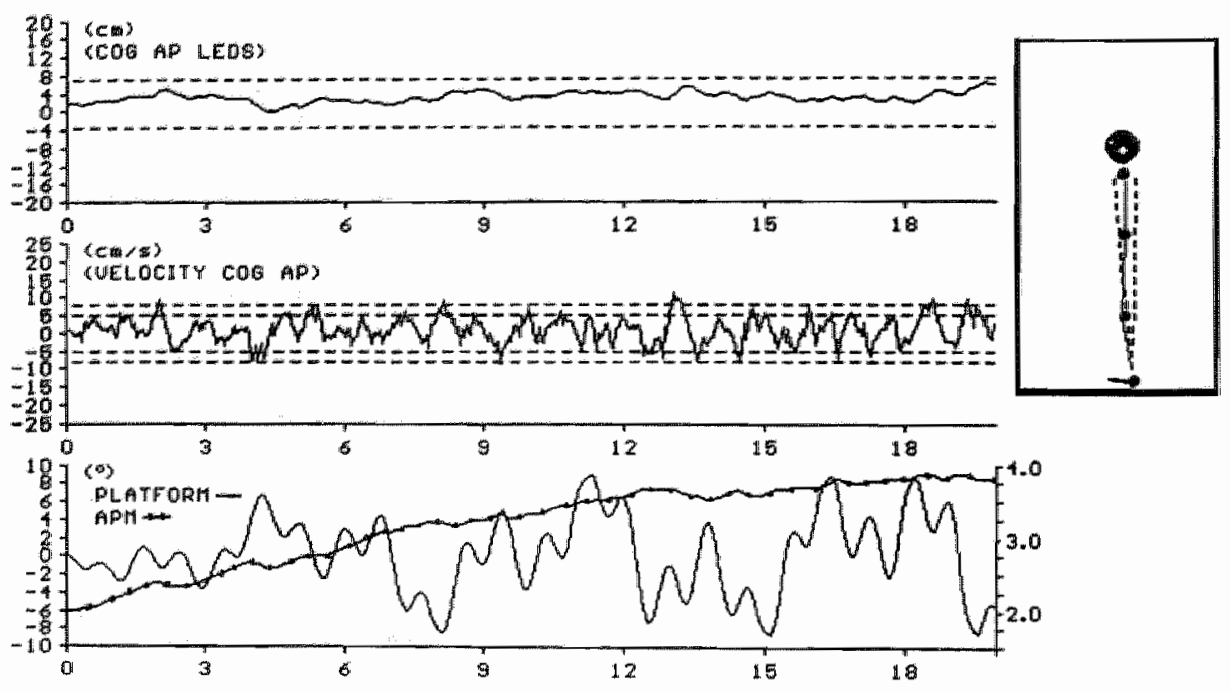

Figure 3. Display of the $P C$-monitor during data-acquisition and analysis, using the $I R$-video tracking system. The upper trace displays the calculated $C O G$ displacement in the antero-posterior direction in cm (Y-axis) versus time $(X$ axis) based upon the video tracking system. The middle trace indicates calculated $C O G$ velocity in $\mathrm{cm} / \mathrm{sec}(Y$-axis) versus time ( $X$-axis). The vertical arrows indicate the predefined $C O G$ velocity windows. $f$ Lower limit,$=$ Stable limit, $\downarrow$ Upper limit. The lower trace displays the platform angle (curve --u- left $Y$-axis) and the pseudo-random APM (curve - --, right $Y$-axis) versus time ( $X$-axis.). On the right a schematic representation of the examined subject with IR markers is depicted. The movements of the markers and the position of the COG are monitored real time (-infrared light markers on the body, o infrared light marker on the head). After fimishing the test the registered subject's movements can be reconstructed and observed in detail by moving the cursor horizontally through the upper trace. 
The output of the angular rate sensor and the video system showed a comparable pattern, but were clearly different from the output of the force plate if the subjects were asked to make vigorous body movements (experiment if, figure $2 \mathrm{f}$ ). In several experiments we observed that many subjects tend to re-arrange the pressure distribution of the feet on the platform when the platform or the body moved with frequency components exceeding $0.4-0.5 \mathrm{~Hz}$ (FFT spectral analysis). In the latter case the force platform did not reveal reliable information any more about the real movement of the $\mathrm{COG}$, as could be judged from the video recondings. So the similarity of the curves shown in figures $2 \mathrm{~d}$ and $2 \mathrm{e}$ suggests that all three detection techniques give a reliable estimate of body sway under static conditions (with all frequency components of the body movements below $0.2 \mathrm{~Hz}$ ). However, in contrast, the three traces of figures $2 \mathrm{a}, 2 \mathrm{~b}$, $2 c$, and $2 \mathrm{f}$ all show remarkable differences. Analysis of the video registration -frame by frameallowed us the following interpretation of the results.

In figures $2 \mathrm{a}$ and $2 \mathrm{c}$ no substantial COG movement does occur despite the misleading output of the force transducers and the angular rate sensor respectively. In figure $2 \mathrm{~b}$ substantial $\mathrm{COG}$ movements occur although the angular rate sensor does not detect the movement accurately. In figures $2 \mathrm{~d}$ and $2 \mathrm{e}$ all three detection techniques give a reliable estimate of body sway (static conditions). In figure $2 \mathrm{f}$, the force transducers most likely through the detection of reaction forces suggest COG movements that do not really occur.

Our conclusions with respect to the use of force transducers in posturography are therefore in line with a recent paper by Witte and Recknagels (14) which states that the information on the spatial location of the centre of pressure provided by the force plate permits conclusions to be drawn about the regulation of the 'sway' of the COG only if additional information on the acceleration situation of the body is available. Otherwise the systematic error of the measurement exceeds the amount of the true "sway" even for physiological cyclic movement frequencies of $0.5 \mathrm{~Hz}$ or more (14). So the value of dynamic posturography using force platforms should be considered in line with the limited accuracy of the method to detect the COG position under dynamic conditions.

Throughout these experiments it became clear that the limitation of the detection technique using angular rate sensors is twofold. First, the poor signal to noise ratio and small but relevant drift of angular rate sensors do not allow an accurate detection of the position of the body segment to which the sensor is attached. Second, an accurate estimate of COG velocity is only possible if multiple angular rate sensors are applied in association with a biomechanical model (similar to the video technique) because the COG position is affected by separate movements of many parts of the body.

The similar consideration holds for application of imaging techniques. Detection of head and or shoulder position alone as is done by the cranio-corporography is not sufficient to detect the COG position accurately. Therefore multiple markers should be attached to the body as is already routinely done in gait analysis. However, a draw back of such a video tracking technique, although judged successful in this study, is comprised in the biomechanical model and the associated anthropometric parameters needed to calculate the precise $\mathrm{COG}$ position. These underlying assumptions may introduce considerable systematic errors, which limits the absolute accuracy. Application of more refined and detailed biomechanical models, that are under development by many researchers in the field of posture and gait, might reduce but most likely not fully eliminate these errors. However, based upon the above mentioned observations and the results of experiment 1 , we 
conclude that the video tracking method produced the most accurate and reliable estimate of the COG movement, specially in case of substantial body movements at frequencies above $0.2 \mathrm{~Hz}$. Also, it appeared to be the only method that signals absolute body position (angle) and is optimally suited for direct platform control. Another advantage of the detection of position of the various body segments separately is that an accurate analysis of balance control strategies will also be possible.

\section{Balance control near the limit of stability}

Using the video tracking measuring technique, balance control of all subjects could be quantified in a reproducible way in terms of TPM. Inter-individual variability was clearly larger than the test-retest variability, which supports the idea that balance control abilities indeed differ substantially from individual to individual and that the method introduced here is sufficiently sensitive to detect these differences. The TPM score correlated well with the subject's own judgement of balance capabilities in daily life. This was in contrast with the findings of the routine stabilometry that showed normal and not-significantly different balance control in all $\mathbb{1}$ subjects, irrespective their own judgement of their own balance control. capabilities.

However, despite the optimal detection technique used and the good reproducibility, some problems did occur and critical considerations have to be made. First, the test time allowed to evaluate the subjects balance capabilities was limited to about 20 seconds as most subjects reported loss of concentration and fatigue at a test duration exceeding 20 to 30 seconds resulting in large fluctuations of APM and TPM and poor reproducibility. This observation was confirmed in subsequent studies with highly motivated, well-trained subjects. As a consequence, using this method, it will not always be possible to discriminate between healthy active and moderately active subjects: most healthy subjects will show a steady incline of APM from 2.0 to $6.0 \mathrm{dg}$ with a TPM of 4.0 . We observed in the pilot study that changing the initial value or rise time of APM affected the absolute value of TPM. However, a minimal initial APM of $2 \mathrm{dg}$ is always required to obtain a sufficient challenging condition that is clearly different from the classical Romberg test. In contrast, in the moderately active and nonactive subjects, making the test condition more difficult by a higher initial APM value generally resulted in a longer, and more variable, initial adaptation period and lower reproducibility of TPM. Also, a steeper rise time of APM (e.g. $0.6 \mathrm{dg} / \mathrm{s}$ ), or a smaller (e.g. 5-7 $\mathrm{cm} / \mathrm{s}$ ), lower (e.g. 3-6 cm/s) velocity or smaller position (e.g. $2 \mathrm{dg}$ ) window resulted in frequent oscillations of APM and many sub plateaux without reaching an ultimate plateau near the end of the test. In subsequent studies, we observed that many patients could not cope with these difficult conditions at all and showed a steady decrease and many oscillations of APM with poor reproducibility. Only the well-trained subjects could cope with these more challenging conditions: they either reached no plateau (oscillations in case of very small and low velocity and position windows) or they showed a steady increase of APM to the theoretically maximum. Under these challenging conditions repetition of the experiments in all active and some moderately active subjects showed that they steadily improved TPM to the maximum.

So, the experimentally selected parameters (initial $\mathrm{APM}=2 \mathrm{dg}$, rise time 0.004 per $20 \mathrm{~ms}$, COG position within $3.125 \mathrm{dg}$ forward or backward) are optimal to achieve reproducible results in

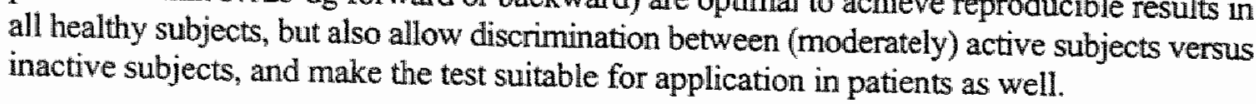


This study was limited to an evaluation of measuring techniques and balance control related to anterior-posterior body sway. However, with some minor technicall adaptations, the video technique is very suitable to monitor multi-segmental body movements in $3 \mathrm{D}$ and allow study of balance control in more detail. We are currently working on this aspect.

\section{Conclusions}

1. Detection of body sway with force platforms or a single body fixed angular rate sensor under dynamic conditions is not reliable.

2. Detection of the position of all relevant body segments is a minimal requirement to reconstruct the $C O G$ position accurately at various conditions.

3. Detection of balance at challenging conditions offers perspectives for the development of equipment to quantify balance control in a reproducible way. 


\section{References}

1. Terekhov Y. Stabilometry and some aspects of its applications-a review (1976). Biomed. Eng. 11:12-15.

2. Romberg MH. Manual of nervous diseases of man (1853). London: Sidenham Society: 935-941.

3. Black FO. Vestibulospinal function assessment by moving platform posturography (1985) Am. J. Otol.: 39-46.

4. Norré ME. Posture in otoneurology (1990). Acta Oto-Rhino-Laryngol. Belg. 44:355364.

5. Norré ME. Contribution of a posturographic six-test set to the evaluation of patients with peripheral vestibular disorders (1992). J. Vestibular Res. 2:159-166.

6. Seidel $H$, Brauer $D$, Bastek $R$, Issel $L$. On the reproducibility and changes of stabilograms in investigations with long-term performance (1978). Agressologie 19(b): 93-95.

7. Roberts TDM. Neurophysiology of postural mechanisms (1978). $2^{\text {nd }}$ edition. London: Butterworths.

8. Henriksson $\mathrm{NG}$, Johansson $\mathrm{G}$, Olsson LG, Ostlund $\mathrm{H}$. Electric analysis of the Romberg test (1966). Acta Oto-Laryngol. Suppl. 224:272-279.

9. Taguchi K. Spectral analysis of body sway (1977). ORL. J. Otorhinolaryngol. Rel. Spec. 39:330-337.

10. Stockwell CW. Posturography (1981). Otolaryngol. Head Neck Surg. 89:333-335.

11. Chan CWY, Kearny RE, Melvill Jones G. Tibialis anterior responses to sudden ankle displacements in normal and Parkinsonian subjects (1979). Brain Res. 183:303-314.

12. Diener HC, Ackermann H, Dichgans J, Guschlbauer B. Medium and long latency responses to displacement of the ankle joint in patients with spinal and central lesions (1985). Electroencephalogr. Clin. Neurophysiol. 60:407-416.

13. Nashner LM, Peters JF. Dynamic posturography in the diagnosis and management of dizziness and balance disorders (1990). Neurol. Clin. 8:331-349.

14. Witte $H$, Recknagel $S$. Posturography employing movement analysis: physical considerations (1997). Biomed. Tech, 42:280-283.

15. Spaepen AJ, Fortuin JM, Willems EJ. Comparison of movements of the centre of gravity and centre of pressure in stabilometric studies (1977). Agressologie 18:109. 113.

16. Di Fabio RP. Sensitivity and specificity of platform posturography for identifying patients with vestibular dysfunction (1995). Phys. Ther. 75:290-305.

17. Konijnenberg JJ. Visuo-vestibular interaction (1993). Thesis Maastricht University, The Netherlands.

18. Kingma $H$, Gulikers $H$, Jong I de, Jongen $R$, Dolmans $M$, Stegeman $P$. Real time binocular detection of horizontal, vertical and torsional eye movements by an infra-red video eye tracker (1995). Acta Oto-Laryngol. 520:9-15

19. Hufschmidt A, Dichgans J, Mauritz KH, Hufschmidt M. Some methods and parameters of body sway quantification and their neurological applications (1980). Arch. Psychiatr. Nervenkr. 228:135-150.

20. Njiokiktjien C. Statokinesimetrische registratie van het houdingsevenwicht (1971). Thesis University of Amsterdam, The Netherlands. 
21. Snijders CJ, Verduin M. Stabilograph, an accurate instrument for sciences interested in postural equilibrium (1973). Agressologie 14C:15-20.

22. Black FO, Wall CIII. The Vestibulospinal stability test: normal limits (1977). Trans. Am. Acad. Ophthalmol. Otolaryngol. 84:549-560.

23. Winter DA. Biomechanics of human movement (1979). New York: Wiley \& Sons. 4364. ISBN: 0-471-03476-2. 
Chapter 3

\section{Balance control near the limit of stability at various sensory conditions in healthy subjects}

Co-authors: M. Dolmans, I. de Jong and H. Kingma

A revised form of this chapter has been submitted for publication. 


\section{Abstract}

Much research has been done to analyse the impact of the various sensory sub-systems towards balance control. A major problem of most balance tests used clinically is the limited reproducibility due to habituation, fatigue and impact of the subject's instructions, motivation and the pre-knowledge of the stimulus conditions. These aspects hinder a quantitative comparison between successive tests with different sensory conditions. Recently an alternative measuring system was developed to evaluate the postural system near the individual limit of stability where the test subjects are forced to perform optimally to prevent a fall (17). This approach resulted in a good reproducibility that might allow an accurate comparison of the various techniques used to disturb the input of the sensory subsystems. In the study presented here, this new technique is applied in 97 healthy subjects to explore the perturbation effect upon balance control of sway referencing the supporting platform, of sway referencing the visual surround, of closing the eyes, of vibration of the Achilles tendon and chilling the foot soles. Balance control -on average- was maximally affected by closure of the eyes (decrease of performance with about $37 \%$ ) and by vibration of the Achilles tendons (about $21 \%$ ). The other perturbation techniques applied were less effective. The combination of vibration and a sway-referencing platform was the most effective technique used to disturb the proprioceptive input. The effect of chilling the foot soles (5 minutes) was not very effective; longer cooling as reported previously by Magnusson et al (7) was badly appreciated. Combination of perturbation techniques was often more effective but was not merely a linear summation of the effect induced by the techniques applied separately. Combination of closure of the eyes with a sway referenced platform and vibration was the most effective perturbation technique of balance control (56\%). The data suggested that vision contributes maximum $37 \%$, propriocepsis minimum $26 \%$ and labyrinths maximum $44 \%$ to balance control in healthy subjects, and that depending on the available sensory information and acquired balance control strategies, weighting factors of the sensory inputs may vary within these margins. However, individual data suggested that the contribution of the sensory inputs toward balance control vary considerably between individuals, which might limit the clinical relevance of this type of dynamic posturography.

\section{Introduction}

Postural instability is a common problem among patients with vestibular disorders. Much research has been done to analyse the impact of the various sensory subsystems towards balance control in order to get more insight in the processes of sensory substitution and central compensation, and to optimise revalidation. In the currently frequently applied posturography test, 'the sensory organisation test' (SOT), the proprioceptive and visual input to the balance control system indeed can be manipulated to evaluate their contributions. Body sway in the SOT is evaluated in six sensory conditions: 1. stable platform, eyes open; 2 stable platform, eyes closed; 3 stable platform, sway referenced visual surround; 4 sway referenced platform, eyes 
open; 5 sway referenced platform, eyes closed; 6 sway referenced platform and visual surround. In each condition an equilibrium score is calculated by comparing the angular difference between the person's calculated maximum anterior to posterior body centre of gravity (COG) displacement relative to a theoretical maximum displacement. In the SOT, the proprioceptive sensory input is assumed to be affected by a technique called sway referencing $(1,2)$ where the supporting surface moves according to the subjects own anterior-posterior movements. Shumway-Cook and Horak (3) and many others used a comparable technique by placing the patients on a support surface covered with foam rubber. These techniques make the contribution of the foot-ankle proprioception and the pressure receptors in the feet less adequate $(4,5)$. Other techniques used to disturb the proprioceptive receptors during posturograply are vibration of the calf muscles (6) or Achilles tendons, or chilling the foot soles $(7,8)$. Vibration of the calf muscles (6) or Achilles tendons induces instability and an illusion of not existing body movements. The vibration induced body sway is thought to be created by activation of the secondary endings of the muscle spindles, resulting in misleading information concerning the actual muscle length $(6,9)$. Chilling the foot soles appears to enhance body sway too and is believed to anaesthetise the pressure receptors of the foot $(7,8)$. Although it is possible to abolish the visual input to the balance control system simply by closing the eyes $(5,10)$, the previously mentioned SOT incorporates also a visual sway referencing technique (also called stabilised vision) where the visual surround is moved according to the subjects own movements. The rational behind this addition is that the balance control system in some vestibular deficient patients is believed to respond inappropriately to conflicting or inaccurate visual inputs (11). Less sophisticated but comparable techniques are described where body sway is examined in subjects wearing a head mounted visual conflict dome or special designed goggles (12).

However, despite this intensive research, it is still not clear which stimulus is the most effective in disturbing the proprioceptive and visual inputs towards balance control. This aspect complicates a quantification of the impact of visual and proprioceptive input to balance control in the individual. One of the problems encountered is that any effort to determine the contribution of any sensory input to postural stability is hampered by the fact that stabilisation and orientation of the head and body in space depends on learning and habituation processes and on a complex integrative processing of vestibular, visual and proprioceptive-somatosensory inputs (13). The complex, most likely non-linear, integrative processes complicate the interpretation of manipulation of the sensory inputs. Habituation, fatigue and subject's instruction, motivation and preknowledge affect the reproducibility of most balance tests. Specially the latter aspect introduces a methodological problem as the poor reproducibility hinders a quantitative comparison between successive tests with different sensory conditions. As a consequence sensitivity and specificity of for example the SOT are subject of debate $(14,15,16)$.

Recently an alternative measuring system was developed to evaluate the postural system near the individual limit of stability where the test subjects are forced to perform optimally to prevent a fall. During each test, the amplitude of the platform movement was maximised automatically as long as the body movement was in 
accordance with pre-set $C O G$ velocity and $C O G$ position limits. The average platform movement tolerated during the test is then used as a measure for balance control. This approach resulted in a good reproducibility that might allow an accurate comparison of the warious techniques used to disturb the input of the sensory subsystems. In this study, this new technique is used to document normal values of balance control and to explore the extent and reproducibility of the effect of various perturbation techniques upon balance control in 97 healthy subjects.

\section{Subjects}

After informed consent 97 healthy subjects ( 50 females, 47 males) participated in the study. Age ranged from 17 to 68 years (females 19 to 68 , males 17 to 66 ) with a mean age of 30.7 years (females 32.3 , males 29.0 ). Only subjects were included whom showed a normal oto-neurological status and in which, standard vestibular testing (spontaneous, gaze, saccades, optokinetics, smooth pursuit, torsion swing, and calorics) revealed no abnormalities. Also body sway velocity, body sway area, as well as the associated Romberg coefficients of all subjects as revealed by computerised stabilometry, had to be within the normal limits. The subjects had no history of neurologic or musculo-skeletal problems that would affect their balance and orientation. They had no history of episodes of dizziness or underwent any ear operation and were not taking any medication at the time of testing. Only subjects were included whom considered themselves as physically moderately active (incidental but no regular sport activities).

\section{Methods}

The experimental set-up composed of a high resolution $(<0.1 \mathrm{~cm})$ home built real time infrared (IR)-video body movement tracking system, a dynamic force plate (T-Post, Jaeger-Toennies GmbH, Wuerzburg, Germany) provided with a device for cooling the foot soles (Instrumental Service Maastricht University "ISMU", The Netherlands), a dual Achilles" tendon vibrator (ISMU, The Netherlands), a dynamic slide projection system (ISMU, The Netherlands and Jaeger-Toennies GmbH, Wuerzburg, Germany, patent PT1265390) and a modified Nystagliner system (Jaeger-Toennies GmbH, Wuerzburg, Germany) for data-acquisition (set at $50 \mathrm{~Hz}$ per channel) and stimulus control (platform, slide projection, vibrators).

The $50 \mathrm{~Hz} I R$-video tracker system consisted of a video camera focused on the left side of the subjects body. The camera imaged the position of five active IR markers fixed to the ankle, knee, hip and shoulder joints and near the axis of head nodding. The IR marker positions ( $x, y$ in the anterior-posterior plane) were detected in the video frame, grabbed by a personal computer (PC) and subsequently transferred to the dataacquisition PC (Nystagliner). A biomechanical model $(17,18)$ was used to calculate the position and velocity of the $C O G$ in the anterior-posterior plane based upon the marker positions detected. The delay between actual body movement and the calculated position of the COG, was always less than $13 \mathrm{msec}$. Body sway velocity was calculated by 
differentiating the digitised position signal ( 3 points).

The data-acquisition PC also controlled the dynamic platform that could rotate up to 9 $\mathrm{dg}$ maximum with a maximum angular velocity of $50 \mathrm{dg} / \mathrm{sec}$. and maximum angular acceleration of $1400 \mathrm{dg} / \mathrm{sec}^{2}$ (maximum delay $18 \mathrm{msec}$.). The rotation axis of the platform coincided with the axis through the ankle joints.

The dynamic slide projector imaged a random dot pattern on a transview screen (width $3.20 \mathrm{~m}$., height $2.40 \mathrm{~m}$.), $50 \mathrm{~cm}$. in front of the subject. The slide was projected slightly out of focus for optimal visual impact. Both slide position and orientation, and the focal length of the projection lens was controlled by four computer driven synchro motors (maximum delay $2 \mathrm{~ms}$ ). As a consequence the image could rotate, translate and zoom in, zoom out to 'counteract' any body or head motion relative to the projection screen, or to mimic any motion of the visual reference frame. Besides the event and time control of the test procedures, the software was able to use 8 signals as an input to produce 8 output signals to control platform rotation (delay $18 \mathrm{~ms}$ ) or slide position (delay $2 \mathrm{~ms}$ ) according to a user defined transfer function.

The vibration was applied through vibrators attached to the right and left Achilles' tendon by an elastic strip. The PC controlled vibrator's DC-motor (Simprop Electronic) housed in a cylinder allowing a certain degree of movement to govern the amplitude of the vibration with a maximum frequency of $250 \mathrm{~Hz}(+1-10 \mathrm{~Hz})$ and a maximum amplitude of $0.2 \mathrm{~cm}$.

The cold supporting surface consisted of a metal box filled with ice-water (about 1-3 $\left.{ }^{\circ} \mathrm{C}\right)$, specially designed to be placed on the dynamic platform without affecting its movement. It must be marked that the position of the rotation axis was affected by this procedure, but not taken into consideration with respect to the calculations of performance.

\section{Experiments}

\section{General aspects}

In all experiments subjects were asked to stand as stable as possible on the dynamic platform, bare feet, arms folded at the waist resting on the frontal part of the body, looking straight ahead, facing the slide projection screen without leaning forward or backward during each test period of 20 seconds. Temporal vision was blocked by special goggles. Additionally, all subjects wore a headphone supplied with soft, not distracting music to prevent spatial orientation on auditory cues.

COG position and velocity was continuously measured by means of the video system. The basic platform movement consisted out of 3 mutual harmonically unrelated frequencies $(0.16,0.42,1.15 \mathrm{~Hz})$ with an initial sum of all three amplitudes of $2 \mathrm{dg}$. During the experiment the amplitude of the platform movement (APM) increased automatically in time with $0.004 \mathrm{dg}$ per $20 \mathrm{~ms}(0.2 \mathrm{dg} / \mathrm{s})$ as long as COG velocity was below $5 \mathrm{~cm} / \mathrm{sec}$. APM was fixed when the subject could maintain the COG velocity within the range from 5 $-8 \mathrm{~cm} / \mathrm{sec}$. APM automatically decreased with $0.004 \mathrm{dg}$ per $20 \mathrm{~ms}(0.2 \mathrm{dg} / \mathrm{s})$ when COG velocity exceeded $8 \mathrm{~cm} / \mathrm{sec}$. APM also decreased whenever $50 \%(3.125 \mathrm{dg})$ of the 
maximum angle $(6.25 \mathrm{dg})$ of forward or backward leaning ("the cone of stability" reached irrespective the actual body sway velocity, to prevent the subject from falling. Balance control was expressed in terms of the tolerated platform movement 'TPM' quantified as the average APM tolerated during the 20 seconds test duration. With an initial APM of $2 \mathrm{dg}$ and a maximum increase of $0.2 \mathrm{dg} / \mathrm{s}$ this resulted in a maximum APM of $6 \mathrm{dg}$ after $20 \mathrm{sec}$. and a maximum TPM of $4 \mathrm{dg}$. The effect of manipulation the sensory inputs upon the TPM was studied by performing the experiments described below.

Experiment 1. pseudorandom movement (prm)

The subject's base of support was destabilised by the pseudo random rotation of the platform alone during normal vision (projection of a random dot pattern).

Experiment 2. pseudorandom destabilisation with a sway referenced platform (srp) Subjects were tested similar as described in experiment 1 , however -besides the basic pseudorandom movement- the platform angle moved also according to the body sway angle relative to the vertical with a positive feed back gain of 0.5 .

\section{Experiment 3. pseudorandom destabilisation with vibrators (vib)}

Subjects were tested similar as described in experiment 1 with activated vibrators attached to the right and left Achilles' tendon.

\section{Experiment 4. pseudorandom destabilisation with cooled foot soles (col)}

Subjects were tested similar as described in experiment 1 , however the supporting surface was equipped with a metal box filled with ice water. Prior to this test, the subjects were seated on a chair, the feet placed on the cooling-device (mounted on the rotating platform) for 5 minutes. After the feet were pre-chilled, the subjects were asked to stand up and the actual measurement took place immediately thereafter.

Experinent 5. pseudorandom destabilisation with a sway referenced visual image (srs) Subjects were tested similar as described in experiment 1 , however the projected image of the slide moved according to the posterior-anterior sway of the COG (positive feed back gain of 1.0).

Experiment 6. pseudorandom destabilisation with eyes closed (ec)

Subjects were tested similar as described in experiment 1 , however with eyes closed to prevent any visual orientation.

Experiment 7. pseudorandom destabilisation with a sway referenced platform and visual image (srpuesrs)

Subjects were tested similar as described in experiment 2 and 5 combined.

Experiment 8. psetdorandom destabilisation with eyes closed and a sway referenced platform (ectesrp)

Subjects were tested similar as described in experiment 2 , however with eyes closed to prevent any visual orientation. 
Experiment 9. pseudorandom destabilisation with a sway referenced platform and vibrators (srp\&vib)

Subjects were tested similar as described in experiment 2, however also activated vibrators were attached to the right and left Achilles' tendon.

Experiment 10. pseudorandom destabilisation with eyes closed, a sway referenced platform and vibrators (ec\&srp\&vib)

Subjects were tested similar as described in experiment 9, however with eyes closed to prevent any visual orientation.

All subjects $(n=37)$ participated in experiment 1 to 8 . In 13 subjects all 8 experiments were performed twice to investigate reproducibility. Eleven subjects ( 6 females and 5 males) underwent also experiment 9 and 10.

\section{Data-analysis}

The APM during each experiment was recorded as a function of time and TPM was calculated. To evaluate the effect of perturbation, the TPM obtained in each experiment was related to the TPM calculated in experiment 1 and the TPM obtained at all other sensory conditions. Statistical analysis (Friedman and Wilcoxon Matched Pairs Signed Rank Test) was performed using commercially available software (PCSPSS version 7.52 for Windows).

\section{Results}

The results of all experiments obtained in one healthy subject are illustrated in figures 1,2 and 3. The effect of perturbation of the propriocepsis (srp, vib, col and srp\&vib) upon balance control is shown in figure 1. Similarly figure 2 shows the effect of perturbation of vision (ec, srs) and figure 3 shows the effect of simultaneous perturbation of propriocepsis and vision (srp\&s, ec\&srp and ec\&srp\&vib) upon balance control. In 13 subjects, all 8 experiments were performed twice to investigate reproducibility. Reproducibility varies from 4.3 to $13.2 \%$ depending on the sensory condition. The mean and standard deviation (S.D.) of the TPM of all subjects at eight different sensory conditions are shown in figure 4 . Figure 5 shows the mean and S.D. of the TPM in the 11 healthy subjects whom underwent in addition experiment 9 and 10. 
PRM

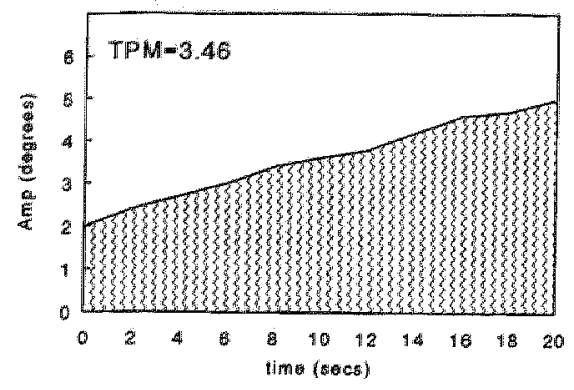

VIB

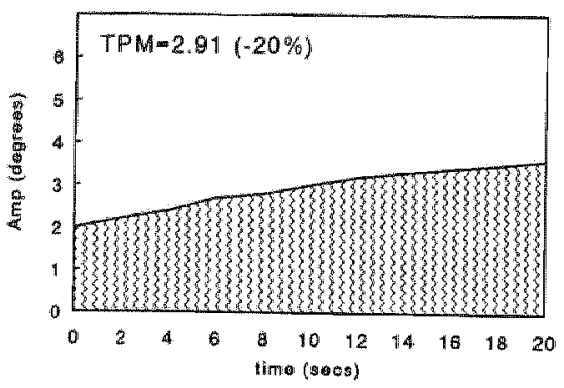

SPP

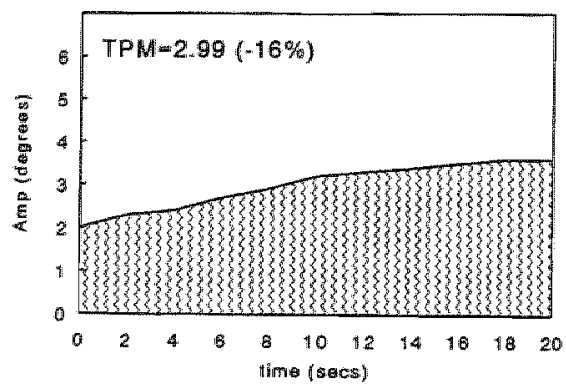

$\mathrm{COL}$

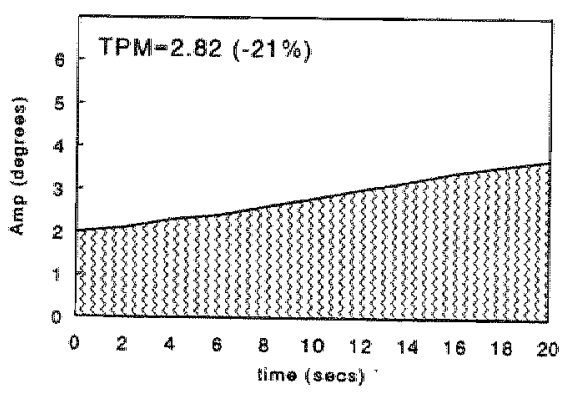

SAP\&VIB

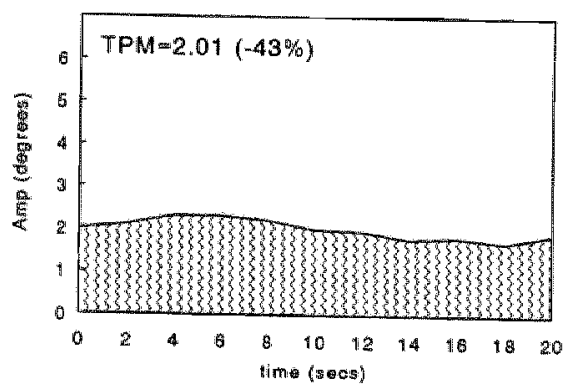

Figure 1. Disturbance of the propriocepsis in a healthy subject. For abbreviations see rext. 
PRM

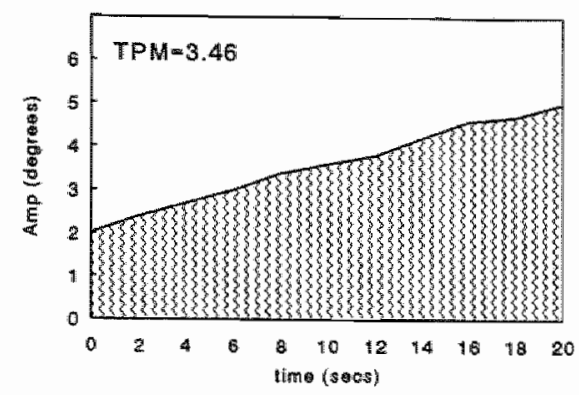

SRS

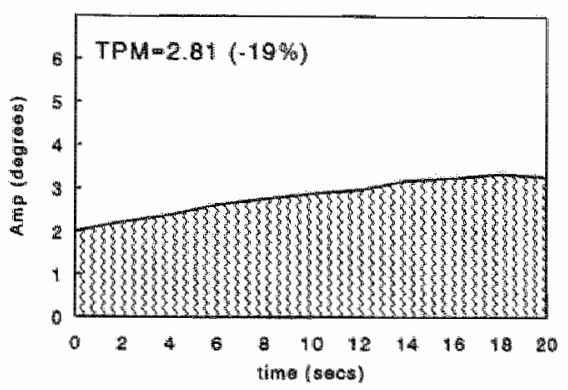

EC

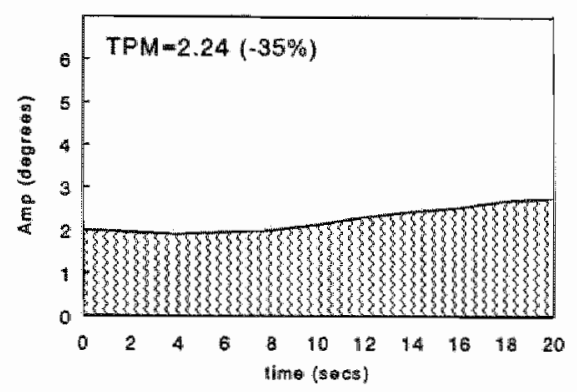

Figure 2. Disturbance of vision in a healthy subject. For abbreviations see text.

Balance control as revealed by the TPM measured in the prm sensory condition measured on awerage 3.55 (S.D. $=0.44, n=97$ ). Compared to the basic prm sensory condition, TPM decreased significantly $(\mathrm{p}<0.01$; paired t-test, $\mathrm{p}<.0005$; Wilcoxon Matched Pairs Signed Ranks Test) at all other nine sensory conditions (see figures. 4, and 5).

Effect of abolishing or misleading the visual cues

Upon closing the eyes, the average TPM decreased from $3.55 \pm 0.44$ to $2.33 \pm 0.52$ and in case of a sway referenced visual surround to $2.69 \pm 0.64$ Figure 4). The ec sensory condition affected posture more effective than the srs sensory condition $(p<.0005)$. 
PRM

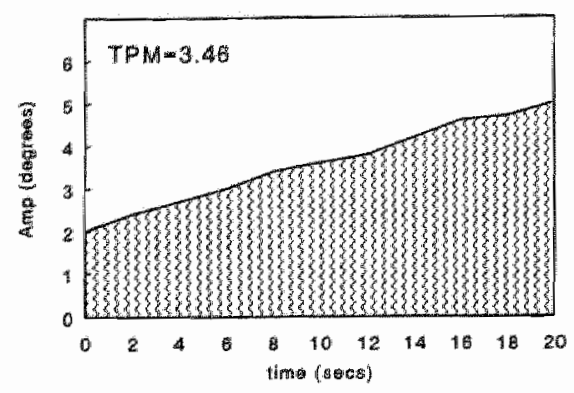

EC\&SAP

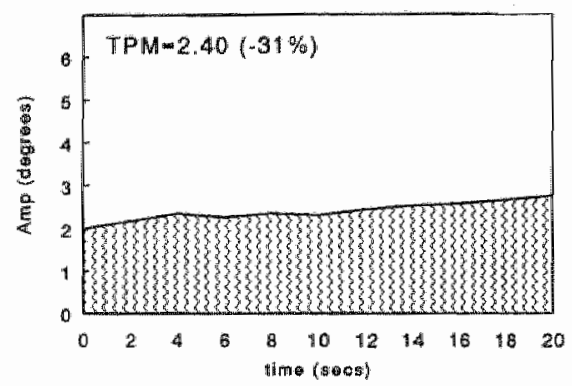

SRP\&S

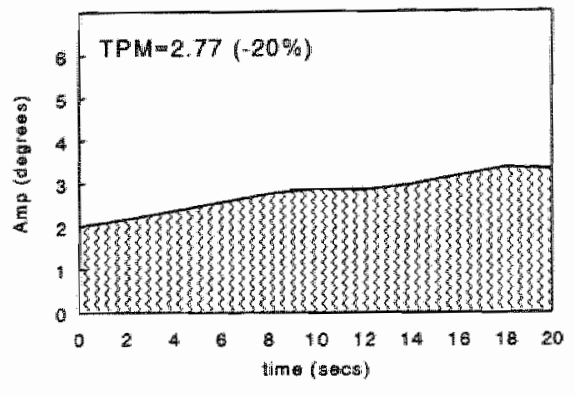

EC\&SRP\&VIG

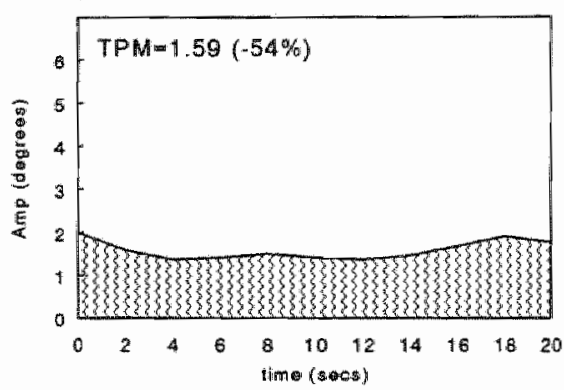

Figure 3. Simultaneous disturbance of the propriocepsis and vision in a healthy subject.

For abbreviations see text.

Effect of misleading the somatosensory-proprioceptive cues

The average TPM decreased under the col sensory condition to $3.17 \pm 0.61$, under the srp sensory condition to $3.05 \pm 0.60$, under the vib sensory condition to $2.74 \pm 0.70$ (figure 4). The srp sensory condition did not disturbed the propriocepsis more than the col sensory condition $(p=0.80)$. The vib sensory condition affected the prm gain significantly more than both the $\operatorname{srp}(\mathrm{p}<0.01)$ and col sensory condition $(\mathrm{p}<0.0005)$.

Effect of combined misleading andior abolishing the visual and somatosensoryproprioceptive cues

The average TPM decreased under the combined srp\&s sensory condition to $2.40 \pm$ 0.90 , under the ec\&srp sensory condition to $1.92 \pm 0.74$ (figure 4). The ec sensory condition affected posture more effectively than the srs sensory condition $(p<0.0005)$ as already mentioned. In accordance with this, the ec\&srp sensory condition was also more effective in 'isolating' the vestibular system than the srp\&s condition ( $p=$ $0.0007)$. 


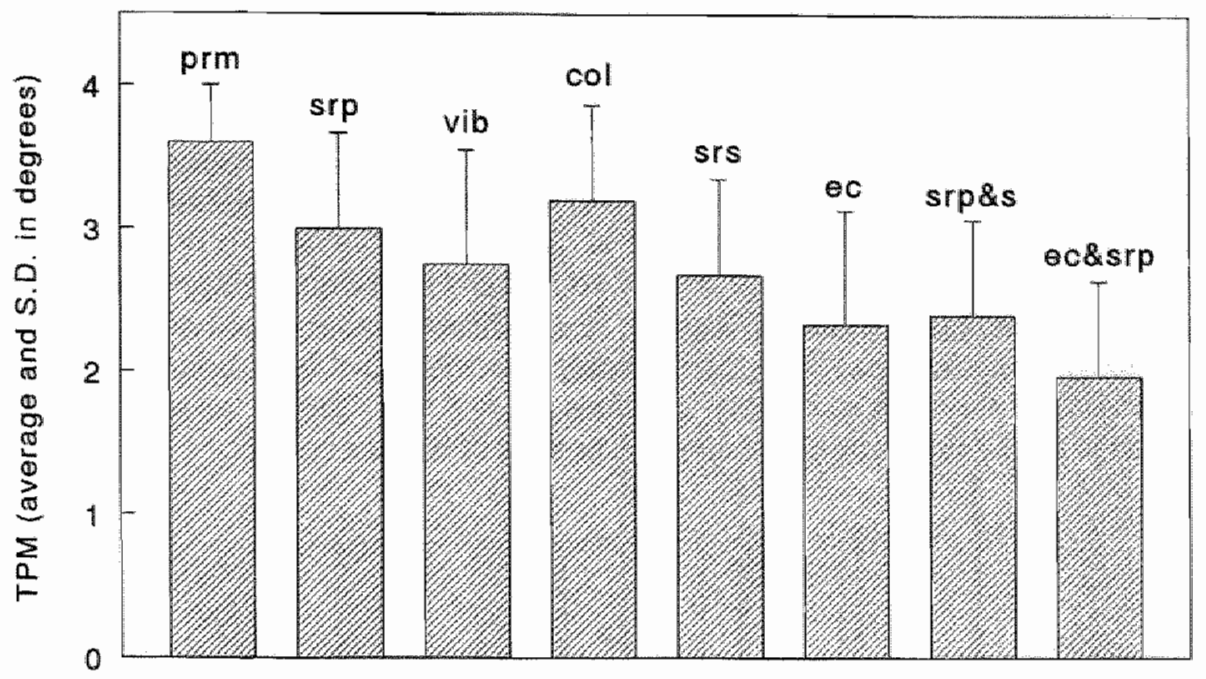

Figure 4. Balance control in 97 healthy subjects. Mean and S.D. (X-axis) of TPM at eight different sensory conditions ( $Y$-axis). For abbreviations see text.

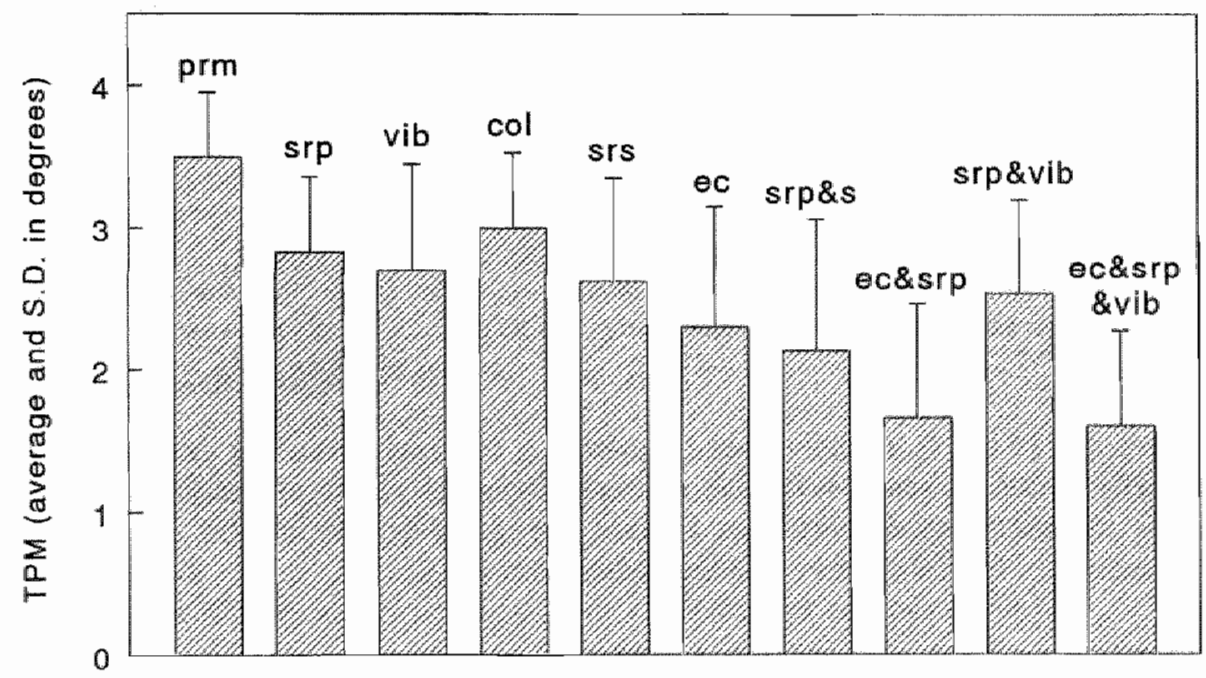

Figure 5. Balance contral in $1 /$ healthy subjects. Mean and S.D. (X-axis) of TPM at ten different sensory conditions ( $Y$-axis). For abbreviations see text. 


\section{Effect of combined use of more than one sensory condition}

The combined use of the sway referenced platform and the vibration, srp\&vib sensory condition (figure 5), had significantly more influence on the somatosensoryproprioceptive system than the col sensory condition $(p=0.03)$ alone, but did not differ significantly from the srp sensory condition $(p=0.23)$ or the vib sensory condition $(p=0.75)$.

The combined use of the srp\&vib sensory condition with the ec sensory condition, the ec\&srp\&vib sensory condition (figure 5), is significantly more effective in "isolating" the vestibular system than the srp\&s sensory condition ( $p<0.01)$, but is not more effective than the srp\&ec sensory condition $(p=0.81)$. The ec sensory condition seemed to have a dominate effect upon the process of balance control.

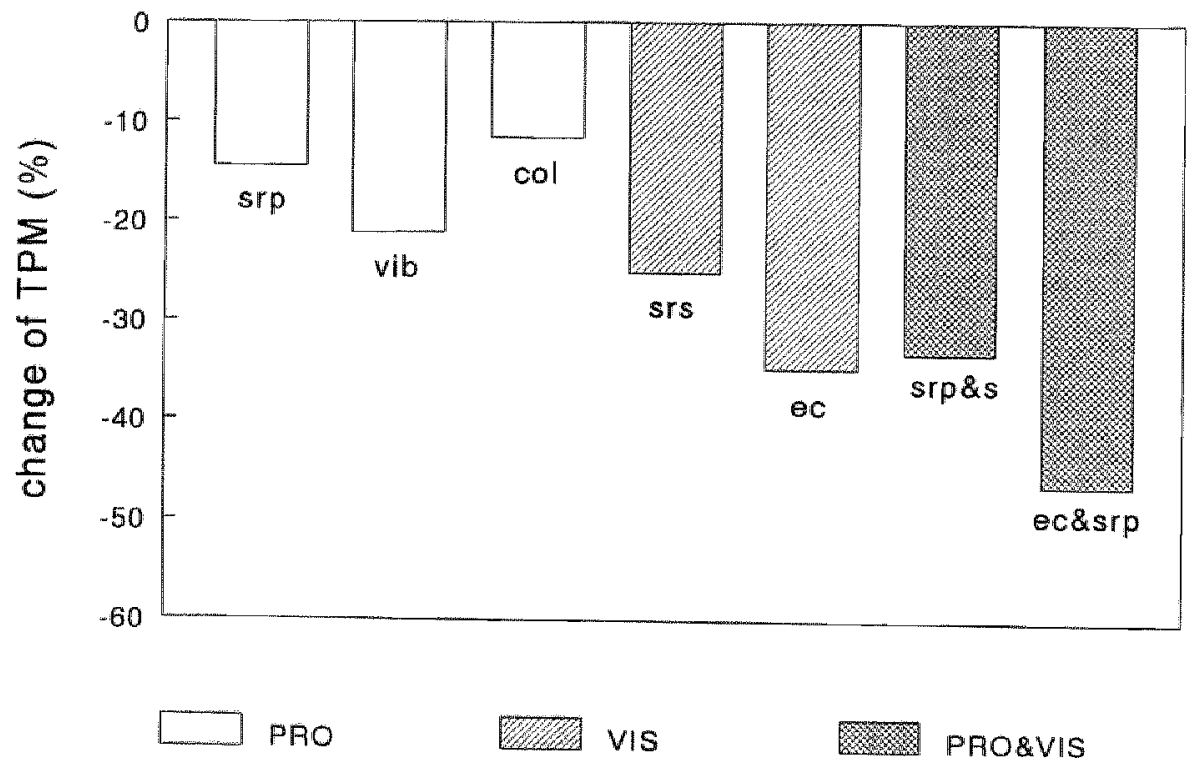

Figure 6. Relative decrease of TPM in 97 healthy subjects by misleading or absent sensory information. srp $=14.49 \%$, vib $=21.14 \%, \mathrm{col}=11.61 \%, \mathrm{srs}=25.15 \%$, $e c=34.79 \%$, srp\&s $=33.21 \%$, ec\& srp $=46.39 \%$.

For abbreviations see text.

Relative sensitivity of the postural system for absent or misleading sensory information Figure 6 shows the relative decrease of balance control by adding misleading (sway referenced, vibration, cooling) or abolishing (eyes closed) sensory information averaged over the population ( $\mathrm{n}=97$ ). In each subject, the gain detected in the prm sensory condition was used as a reference and set at $100 \%$. Balance control was reduced maximum through disturbing the visual sensory input (vis) by $34.79 \%$ (ec). Balance control was reduced maximum through manipulation of the proprioceptive input (pro) by $21.14 \%$ (vib). Balance control was reduced maximum through 
manipulation of visual and propriocepsis simultaneously (vis\&pro) by $46.39 \%$ (ec\&srp).

The relative change of balance control by perturbation varies widely among the subjects. This aspect is illustrated in figure 7 which shows the range $(95 \%$ confidence interval) of the relative change (negative values: decrease; positive values: increase) of balance control by adding misleading (sway referenced, vibration, cooling) or abolishing (eyes closed) sensory information $(n=97)$.

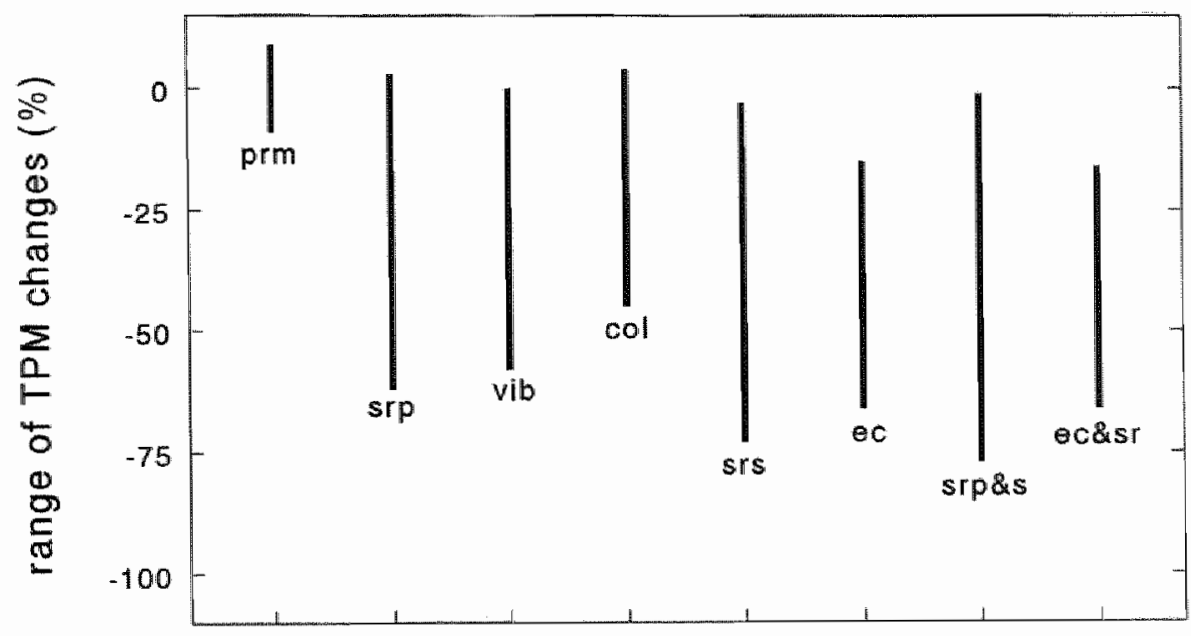

Figure 7. Range ( $95 \%$ confidence interval) of the relative change of balance control in 97 healthy subjects by misleading or absent sensory information (positive values: increase, negative walues: decrease). For comparison the normal test-retest range of TPM in the prm conditions is indicated as well. For abbreviations see text.

Figure 8 shows the average relative decrease of balance control in the 11 subjects that underwent experiment 9 and 10 too. Balance control was reduced maximum through manipulation of the visual input by $36.63 \%$ and through manipulation of the proprioceptive input by $26.1 \%$. Balance control was affected maximum by manipulation of both inputs simultaneously $(55.94 \%)$. 

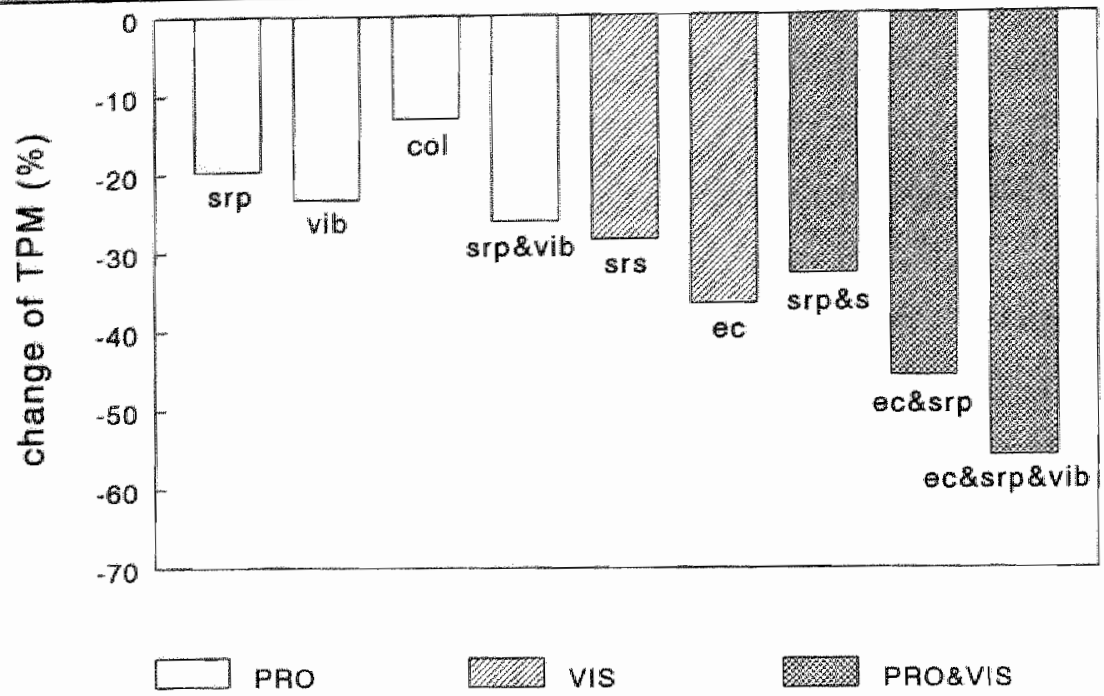

Figure 8. Relative decrease of TPM of 11 healthy subjects by misleading or absent sensory information. $\quad$ srp $=19.65 \%, \quad$ vib $=23.25 \%, \quad$ col $=13.00 \%$, srp\&vib=26.10\%, srs $=28.38 \%, \quad e c=36.63 \%, \quad$ srp\&s $=32.79 \%$ ec\&srp $=45.81 \%$, ec\&srp\&vib=55.94\%.

For abbreviations see text.

\section{Discussion}

The effect of various perturbation techniques upon balance control was evaluated in this study. The effect was quantified near the limit of stabillity, assuming that under these conditions motor control might be more sensitive for any perturbation than at quiet stance and that any subject is forced to an optimal performance. Abolishment or addition of misleading sensory information induced a decrease of balance control in a reproducible way (test-retest variation better then $13.2 \%$ ).

Balance control was maximally affected by closure of the eyes and by vibration of the Achilles tendons (figure 6). On average, the other techniques applied were significantly less effective. Closure of the eyes had clearly significantly more impact on balance control than a sway referenced visual surround. This result is in agreement with the results of the SOT in young healthy subjects (Nashner, personal communications, 1995). Many subjects indicated that it took a short while to identify the inaccurate visual cues, but that they ultimately were able to use it as a reference. One might therefore hypothesise that even "inaccurate" wisual information can be used for orientation relative to the earth vertical.

Vibration of the Achilles tendons appeared to be the most effective method to disturb the somatosensory-proprioceptive contributions to balance control. The combination of vibration and a sway referencing platform was even more effective. Magnusson et al (7) combined the vibration technique with chilling of the foot soles in order to have an intense disturbing influence on the system. In our study the effect of chilling the foot 
soles was not very effective. However, we used a milder cooling technique than reported in Magnusson's study: the feet were chilled 5 minutes prior to the actual measurement, whereas Magnusson chilled the feet 30 minutes prior to the experiment. Long cooling was badly appreciated by our subjects. The vibration technique seems to be an effective, easy and cheap alternative for the sway referencing technique which requires a motor driven platform, although the latter equipment is obligatory if a measuring technique is used as presented here.

\begin{tabular}{|l|l|l|l|l|l|}
\hline & srp & srs & ec & srp\&s & ec\&srp \\
\hline SOT & $18(30) \%$ & $9(14) \%$ & $8(15) \%$ & $33(52) \%$ & $31(48) \%$ \\
\hline TPM & $14.49 \%$ & $25.15 \%$ & $34.79 \%$ & $33.21 \%$ & $46.39 \%$ \\
\hline
\end{tabular}

Table 1. Decrease of balance control by perturbation near the limit of stability (TPM) and the SOT (upper row: average and, between parenthesis $5^{\text {th }}$ percentiles in 97 healthy subjects aged 17-68 years). For abbreviations see text.

The impact of disturbing the sensory subsystems near the limit of stability is generally larger than observed with the SOT at relative quiet stance (table 1, data extracted from the normative graphs of the Equi-test manual (19), maximum estimates based upon the assumption that the SOT score is 100 in condition 1 ). In the visual conditions (ec and srs) the average effect is 3-4 times bigger at the limit of stability than at quiet stance. Considering the extend of the sensory disturbance, it seems as if condition ec with prm is comparable with condition ectsrp of the SOT. The reproducibility of the SOT measurements (trial 1 to trial 3 change) varies with the sensory conditions. In condition 6 (srp\&s) the average trial 1 to trial 3 change is 9 out of an average score of $69(13 \%)$, but both change as average have wide ranges ( $+/-26.4$ and 48 to 100 , respectively) suggesting a far more larger trial to trial variability. The trial 1 to trial 3 change of the TPM varies with the sensory condition too, but was always below $13.2 \%$.

The combination of perturbation techniques near the limit of stability is rather effective in case of srp\&vib and ec\&srp\&vib (figure 8). However, the effect of perturbation by a combination of techniques is not merely a linear summation of the effect induced by the techniques applied separately.

For example: ec reduces balance control by $34.79 \%$, srp by $14.49 \%$ and srs by $25.15 \%$; however, ec\&srp reduces balance control not by $34.79+14.49=49.28 \%$ but by $45.81 \%$, and srp\&s reduces balance control not by $25.15+14.49=39.64 \%$ but by $33.21 \%$ (figure 5). These differences are significant (Wilcoxon, $\mathrm{p}=0.01$ ). The non linearity is more pronounced in case of the combined perturbation ec\&srp\&wib (separately: $36.63+19.65+23.25=79.53 \%$, but together: $55.94 \%$, see figure 6 ).

This non-linearity is observed too in case of exclusive perturbation of the proprioceptive input (figure 6): vib reduced balance control by $23.25 \%$, srp by $19.65 \%$, the combination of both srp\&evib leads to a reduction by $26.1 \%$ in stead of $42.9 \%$. This behaviour can be explained by many, however not mutually exclusive mathematical models that describe balance control in terms of input-output relations (selective thresholds, saturation effects, interaction between sensory inputs etc.). Despite this 
aspect, it is still valid to conclude that vibration of the Achilles tendons and closure of the eyes are the most effective perturbations of the proprioceptive and visual input in healthy subjects respectively.

Based upon these results one could speculate about the relative contributions of the visual, proprioceptive and vestibular systems to balance control. To do so two fundamental assumptions have to be made. First, one needs to assume that no other than visual, vestibular or proprioceptive sensory modalities contribute. This assumption is still a matter of heavy dispute, as for example the existence of special gravito-receptors in the human body have been postulated (20). Second, one needs to assume that the different techniques used, disturb the visual and/or proprioceptive system completely. This assumption certainly holds for the ec conditions but most likely does not hold for the proprioceptive system: the balance control system will certainly receive relevant input from joints and ligaments all over the body that is not affected by the perturbation techniques applied here. Neverthelless, the following estimate is made to give an indication of the possible relative magnitudes of the various sensory contributions. At first glance, manipulation of the visual system seems to affect balance control to a greater extent than the proprioceptive system (figures. 6 and $8,34.79-36.63 \%$ versus $26.1 \%$ ). Eyes closed combined with a sway referenced platform is effective to "isolate" the vestibular system to some extent in agreement with literature $(21,22)$. The combination of eyes closed with a sway referenced platform and vibration leads to a maximum decrease of balance control of about $56 \%$. The labyrinthine input can therefore be estimated to contribute maximally $44 \%$ to balance control under these conditions, as at least proprioceptive perturbation can be assumed to be far from complete. In summary: these data suggest that -on averagevision contributes maximum $37 \%$, propriocepsis minimum $26 \%$ and the labyrinths maximum $44 \%$ in healthy subjects, and that depending on the available sensory information and acquired balance control strategies, weighting factors of the sensory inputs may vary within these margins.

However, several complications still hinder a straight forward interpretation of these estimates. For example, the impact of propriocepsis on balance control is two-fold First, propriocepsis, together with visual and vestibular input, serve to perceive the spatial orientation of the body to induce appropriate motor action. Second, propriocepsis serves in the feed-back loops of all motor-control systems themselves, and plays an important role on the effector side of the balance control system. As the contribution of the propriocepsis on balance control is studied through detection of motor activity (motion), the question is, whether we can ever discriminate between these two aspects with the current methodologies.

Also, the results discussed so far refer to the evaluation of the averaged values of TMP in the population of 97 healthy subjects. It must be stated that the analysis of the individual data reveal quite different results: the sensitivity for the various perturbation techniques varies widely between the subjects (figure 7). This might be due to differences in motor learning strategies acquired in relation to daily life requirements. This aspect at least hampers a generalisation of the estimate made above and complicates the localisation of deficits of the three sensory sub-systems in patients based upon measurements of balance control. 


\section{References}

1. Black FO, Nashner LM. Vestibulospinal control differs in patients with reduced versus distorted vestibular function (1984). Acta Oto-Laryngol. Suppl. 406:110114.

2. Black FO. Vestibulospinal function assessment by moving platform posturography (1985). Am. J. Otol. Suppl. 3:39-46.

3. Shumway-Cook A, Horak FB. Assessing the influence of sensory interaction on balance: suggestions from the field (1986). Phys. Ther. 66:1548-1550.

4. Norré ME. Posture in otoneurology (1990). Acta Oto-Rhino-Laryngol. Belg. 44:355-364.

5. Norre ME. Sensory interaction testing in platform posturography (1993). J. Laryngol. Otol. 107:496-501.

6. Pyykko I, Hansson GA, Schalen L, Henriksson NG, Wennmo C, Magnusson M. Vibration induced body sway (1983). In: Claussen CF (ed.). Computer in der Neuro-otologie. Proc. Neuro-otogical and Equilibriometric Society, 10:139-155.

7. Magnusson M, Enbom H, Johansson R, Pyykko I. Significance of pressor input from the human feet in anterior-posterior postural control. The effect of hypothermia on vibration-induced body-sway (1990). Acta Oto-Laryngol 110: 182-188.

8. Magnusson M, Enbom H, Johansson R, Wiklund J. Significance of pressor input from the human feet in lateral postural control. The effect of hypothermia on galvanically induced body-sway (1990). Acta Oto-Laryngol. 110:321-327.

9. Pyykko I, Toppila E, Starck J, Aalto H, Enebom H, Siedel H: Computerized posturography: development of stimulation and analysis methods (1986). In: Claussen CF, Kirtane MV (eds.), Vertigo, nausea, tinnitus and hearing loss in cardio-vasucular diseases. Amsterdam: Elsevier Science Publishers, 353-362.

10. Booth JB, Stockwell $\mathrm{CW}$. A method for evaluating vestibular control of posture (1987). Otolaryngol. 86: 93-97.

11. Black FO, Wall CIII, Nashner LM. Effects of wisual and support surface orientation references upon postural control in vestibular deficient subjects (1983). Acta Oto-Laryngol. 95:199-201.

12. Horak FB. Clinical measurement of postural control in adults (1987). Phys. There 67:1881-1885.

13. Sloane $P$, Blazer $D$, George LK. Dizziness in a community elderly population (1989). J. Am. Geriatr. Sac. 37:101-108.

14. Dobie RA, Platform Posturography (Letter; comment)(1996). Arch. Otolaryngol. Head Neck Surg. 122: 1273-1276.

15. Black FO, Homer L. Platform Posturography (Letter; comment) (1996), Arch. Otolaryngol. Head Neck Surg. 122: 1273-1276.

16. Di Fabio RP. Sensitivity and specificity of platform posturography for identifying patients with vestibular dysfunction (1995). Phys. Ther. 75: 290-305.

17. EL-Kahky AM, Kingma H, de Jong I, Dolmans M. Dynamic posturography in healthy subjects: evaluation of COG measuring techniques and individualisation of test conditions. Chapter 2 of this thesis. 
18. Winter DA. Anthropometry. Biomechanics of human movement (1979). 43-64.

19. NeuroCom Int. Inc. Data interpretation manual 'Equitest system', version 4.0 (1991).

20. Mittelstaedt $H$. Interaction of eye-, head-, and trunk-bound information in spatial perception and control (1997). J. Vestibular Res. 7: 283-302.

21. Black FO, Shupert CL, Horak FB. Abnormal postural control associated with peripheral vestibular disorders (1988). Prog. Brain Res. 76:263-275.

22. Kantmer RM, Rubin AM, Armstrong CW, Cummings V. Stabilometry in balance assessment of dizzy and normal subjects (1991). Am. J. Otolaryngol. 12:196-204. 


\section{Chapter 4}

\section{Effect of training on a balance bar upon}

postural control near the limit of stability in healthy subjects: a double blind placebo controlled study 


\section{Abstract}

Recently we developed a new technique to evaluate the postural system near the individual limit of stability with a low test-retest variation. In this double blind placebocontrolled study this technique is applied to evaluate the effect of habituation to the test conditions in 11 healthy subjects (experiment 1) and to explore the effect of specific balance training upon balance control in 56 other healthy subjects (experiment 2). In the first experiment subjects were tested three times on one day. In the second experiment 28 subjects were trained on a balance bar (training group), while 28 subjects performed a pseudo-training (control group). The results show no significant effect of habituation to the test condition or equipment. Training on the balance bar showed a steady improvement with respect to the balancing abilities on the bar from session to session. The subjects in the 'training' and control group reported no clear change of balance control in daily life activities irrespective the significant improvement of performance on the balance bar by the training group. No effect of balance training was observed upon balance control near the limit of stability as quantified by dynamic posturography. The negative test outcome of dynamic posturography correlated well with the absence of any effect of balance training upon dailly life activities but does not correlate with the improvement of performance on the balance bar. We concluded that posturography near the limit of stability is not able to quantify the effect of specific balance training that has no impact on balance control in daily life activities. In contrast, in a previous study, we observed that the test scores obtained by posturography near the limit of stability correlated well with the subjects own judgement of balance capabilities (sports) in daily life. It is suggested that dynamic posturography primary evaluates motor control and less spatial orientation and is not suited to evaluate specific balance control as a reflection of spatial orientation abilities.

\section{Introduction}

Any effort to quantify balance control in a reproducible way is hampered by several aspects (1). First, through active learning processes, subjects habituate to the test conditions. Second, test performance depends on the instruction: what is the subject supposed to do precisely. Third, test performance depends on the subject's motivation. In case of complex motor tasks, subjects tend to perform better and show a steady improvement during successive testing with optimal instruction and motivation. Dynamic posturography is often put forward as a method to quantify the effect of vestibular habituation training upon balance control in patients $(2,3,4,5)$, which related to the previous, rises the question whether posturography evaluates habituation to the testcondition, actual balance control (training aspects included) and/or the subject's motivation.

The normal balance control mechanism in healthy subjects has been reported to show a remarkable stability: in individual responses, no statistically significant day-to-day variation in sway velocity and average displacement as assessed by stabilometry is 
observed and intra-individual variation tends to be small (6). Fatigue seems not to affect stability: fatiguing exercises of the right leg calf muscles induce no change of body sway in healthy subjects standing on the fatigued right leg. Nevertheless, there is an adaptive process active as in contrast, a significant decrease of body sway over time when standing on the nonfatigued leg can be observed (7). Apparently balance control is kept within rather precise limits, irrespective a certain amount of fatigue $(6,7)$. So, without training one might expect a rather constant performance with respect to balance control in static conditions.

In contrast to fatigue, training seems to have an impact on basic body sway as it has been shown that untrained subjects are less stable than dancers, which in turn are less stable than acrobats (8). This suggests that stabilometry could be used to evaluate the impact of training upon balance control in daily life activities (acrobatics, dancing etc). Seemingly in line with this, it was shown that body sway in healthy subjects decreases over a ten-day training session by visualisation of their COG during computerised feedback of their body sway in the anterior-posterior direction (9). Here the training sessions were used to train but also to assess balance control and it might well be that the improvement was primarily due to habituation to the test condition and less due to an improvement of balance control during daily life activities. And indeed, it was shown by others (10) that static balance control itself as revealed by stabilometry was not improved after visual feedback training, despite a significant improvement of the performance during the training exercises. These findings suggest that laboratory equipment only shows an improvement if the type of training is closely related to the type of balance assessment at least regarding (semi-) static measurements of balance control. With this respect dynamic posturography has been reported to be more sensitive. Balance training of elderly that improves walking, balance at one leg stance, remarkably also improved the test scores in the 3 most difficult test conditions of the equitest dynamic posturography (srp, ec\&srp, srp\&s) (11).

Recently we developed a measuring system to evaluate balance control near the individual limit of stability where the test subjects are forced to perform optimally to prevent a fall (12). This approach resulted in a good reproducibility that allowed an accurate comparison of the various techniques used to disturb the input of the sensory subsystems (13). The method is not that much influenced by the subject's motivation as is a major problem in stabilometry, as the responses associated with a poor motivation are easily recognised (12). The latter aspect is an interesting feature as it might allow us to evaluate the effect of balance training on balance control. In this double blind placebo-controlled study, this technique is applied to evaluate the effect of habituation to the test condition and equipment (experiment 1), as well as to measure the effect of training on a balance bar upon balance control (experiment 2).

\section{Subjects}

After informed consent 67 healthy medical students ( 29 males, 38 females) volunteered for participation in this study. The subjects had no history of neurological or musculoskeletal problem that could be expected to affect their balance control and 
spatial orientation capabilities. In addition, they had no history of episodes of dizziness or ear operations and were not taking any medication at the time of testing.

Eleven heal thy subjects ( 5 females, 6 males, 28 - 68 years old) participated in experiment 1 dealing with the effect of habituation to the equipment. All 11 subjects showed a normal oto-neurological status and audiometry, and standard vestibular testing (spontaneous, gaze, saccades, optokinetics, smooth pursuit, torsion swing, and calorics) revealed no abnormalities. All subjects had a moderately active life (no substantial regular sport activities). Body sway velocity, body sway area, as well as the associated Romberg coefficients of all subjects as revealed by computerised stabilometry were well within the normal limits.

Fifty-six healthy medical students ( 24 males, 32 females) volunteered for participation to experiment 2 dealing with the effect of training upon balance control. The subjects were not informed concerning the different training programs used in this study (placebo versus training) apart from some general information, which stated that the training was expected to be effective in improving balance control. Fifty-two out of the 56 subjects judged their balance capabilities as moderate to good, the other four as good. Subjects were randomised into a "training" group and a control group. The training group comprised 28 healthy students (11 males and 17 females, 17 to 25 years of age with a mean age of 20.75 years). The control group comprised 28 healthy students (13 males and 15 femalles, 18 to 31 years of age with a mean age of 21.25 years).

\section{Methods}

The experimental set-up composed of a high resolution $(<0.1 \mathrm{~cm}$.) home built real time infrared (IR)-video body movement tracking system, a dynamic force plate of which the rotation axis coincided with the axis through the ankle joints (T-Post, JaegerToennies GmbH, Wuerzburg, Germany) provided with a device for cooling the foot soles (Instrumental Service Maastricht University (ISMU, The Netherlands)), a dual Achilles' tendon vibrator (ISMU, the Netherlands), a dynamic slide projection system (ISMU, The Netherlands and Jaeger-Toennies GmbH, Wuerzburg, Germany, patent PT1265390) and a modified Nystagliner system (Jaeger-Toennies GmbH, Wuerzburg, Germany) for data-acquisition (set at $50 \mathrm{~Hz}$ per channel) and stimulus control (platform, slide projection, vibrators). The $50 \mathrm{~Hz}$ IR-video tracker system imaged the position of five active IR-markers fixed to the ankle, knee, hip and shoulder joints and near the axis of head nodding. The position and velocity of the centre of gravity of the body (COG) in the anterior-posterior plane based upon the marker positions was detected (12). The dynamic slide projector imaged a random dot pattern on a transview screen (width $3.20 \mathrm{~m}$., height $2.40 \mathrm{~m}$., temporal vision was blocked by special goggles), $0,5 \mathrm{~m}$. in front of the subject. Both slide position and orientation, and the focal length of the projection lens was controlled by four computer driven synchro motors to allow any motion of the observed visual reference frame. The vibration was applied through home built vibrators attached to the right and left Achilles' tendon by an elastic strip. The computer-controlled vibrator's DC-motor (Simprop Electronic) 
housed in a cylinder allowing a certain degree of movement to govern the amplitude of the vibration with a maximum frequency of $250 \mathrm{~Hz}(+/-10 \mathrm{~Hz})$ and a maximum amplitude of $0.2 \mathrm{~cm}$. The cold supporting surface consisted of a metal box filled with ice water (about 1-3 ${ }^{\circ} \mathrm{C}$ ), specially designed to be placed on the dynamic platform without affecting its movement (ISMU). It must be marked that the position of the rotation axis was affected by this procedure, but was not taken into considerations with respect to the calculations of the performance.

\section{Experiment 1. effect of habituation to the equipment}

Balance control was assessed in each subject three times a day, at $9.00 \mathrm{am}, 13.00 \mathrm{p.m}$. and 17.00 p.m., using 8 sensory conditions as described below (see balance control measurement).

\section{Experiment 2. effect of training on a balance bar upon balance control}

\section{Balance training}

\section{Training group}

The training group was trained in the hospital under direct supervision twice per week. The program was meant to optimise the vestibulo-ocular reflex (VOR) as well as the vestibulo-spinal reflex (VSR). First the subjects were asked to shake the head as fast as possible (yaw rotation) while fixating a pseudo-random moving visual target with a maximum of 3 minutes or until they started to feel dizzy. The balance exercises consisted of walking on a wooden bar $(2.5 \mathrm{~m}$. long and $15 \mathrm{~cm}$. wide) for five minutes, step by step, under the following eight conditions (in order of increasing complexity):

1. Eyes open, looking straight ahead; 2. Eyes open, head in retro-flexion; 3. Eyes open, looking to a fixed visual target; 4 . Eyes open, visually tracking a pseudo random moving target; 5 . Eyes closed; 6 . Eyes closed, head shaking in the horizontal plane; 7 . Eyes closed, head in retro-flexion; 8. Eyes closed, head shaking during retro-flexion.

The subjects started to balance on the bar in condition one. If they succeeded in reaching the end of the bar without fall or hesitation, the next condition was tried. In case of a fall or hesitation the previous condition was tried again. Subjects completed fourteen training sessions in total, two times per week.

\section{Control group}

The control group performed a pseudo-training at home. The pseudo-training program for the control group consisted of 10 very slow yaw rotations of the head, to the right and to the left while they were sitting on a chair. The pseudo balance exercise consisted of five slow head rotations to the right or to the left while standing (=yaw rotation).

\section{Balance control measurement}

The progress of balance control in the "training" group was scored after each training 
session by registration of condition ( 1 to 8 ) they could maximally cope with. Balance controll was quantified 1 day before the beginning and one day after finishing the training program and was performed as follows. The examiner (AEK) was not aware of the actual training program performed by the subject.

Subjects were asked to stand as stable as possible on the dynamic platform, bare feet, arms folded at the waist, looking straight ahead, facing the slide projection screen without leaning forward or backward during each test period of 20 seconds. All subjects wore a headphone supplied with soft, not distracting music to prevent spatial orientation on auditory cues. COG position and velocity was continuously measured by means of the video system. The basic platform movement (12) consisted out of 3 mutual harmonically unrelated frequencies $(0.16,0.42,1.15 \mathrm{~Hz})$ with an initial sum of all three amplitudes of $2 \mathrm{dg}$. During the duration of the experiment the amplitude of the platform movement (APM) increased automatically in time with 0.004 degree per $20 \mathrm{~ms}(0.2 \mathrm{dg} / \mathrm{s})$ as long as COG velocity was below $3 \mathrm{~cm} / \mathrm{s}$ (in refs. 12 and 13 this level was set at 5 $\mathrm{cm} / \mathrm{s}$ ). APM was fixed when the subject could maintain the COG velocity within the range from $3-5 \mathrm{~cm} . \mathrm{s}$ (in refs. 12 and 13 this window was set at $5-8 \mathrm{~cm} . \mathrm{s}$ ). APM automatically decreased with $0.004 \mathrm{dg}$ per $20 \mathrm{~ms}(0.2 \mathrm{dg} / \mathrm{s})$ when COG velocity exceeded $5 \mathrm{~cm} / \mathrm{s}$ (in refs. 12 and 13 this level was set at $8 \mathrm{~cm} / \mathrm{s}$ ). APM also decreased whenever $50 \%$ (3.125 degrees) of the maximum angle $(6.25 \mathrm{dg})$ of forward or backward leaning was reached irrespective the actual body sway velocity, to prevent the subject from falling. Balance control was expressed in terms of the tolerated platform movement (TPM) quantified as the average APM tolerated during the 20 seconds test duration. With an initial APM of $2 \mathrm{dg}$ and a maximum increase of $0.2 \mathrm{dg} / \mathrm{s}$ this resulted in a maximum APM of $6 \mathrm{dg}$ after $20 \mathrm{~s}$. and a maximum TPM of 4 degrees. The velocity levels were adapted as compared to refs. 12 and 13 , to allow a substantial improvement after training, because all subjects already reached a TPM near to the maximum value of 4.0 with the velocity setting of refs. 12 and 13, due to their good balance capabilities. Eight different sensory conditions were evaluated.

Pseudorandom movement (prm); the subjects base of support was destabilised by the pseudo random rotation of the platform alone during normal vision (projection af a random dot pattern).

Pseudorandom destabilisation with a sway referenced platform (srp); subjects were tested similar as described in condition prm, however -besides the basic pseudorandom movement- the platform angle moved also according to the body sway angle relative to the vertical with a positive feed back gain of 0.5 .

Pseudorandom destabilisation with vibrators (vib); subjects were tested similar as described in condition prm with activated vibrators attached to the right and left Achilles' tendon.

Pseudorandom destabilisation with cooled foot soles (col); subjects were tested similar as described in condition prm, however the supporting surface was equipped with a metal box filled with ice water. Prior to this test, the subjects were seated on a chair, the feet placed on the cooling-device (mounted on the rotating platform) for 5 minutes. After the feet were pre-chilled, the subjects were asked to stand up and the actual measurement took place immediately thereafter.

Psetudorandom destabilisation with a sway referenced visual image (srs); subjects were 
tested similar as described in condition prm, however the projected image of the slide moved according to the posterior-anterior sway of the COG (positive feed back gain of 1.0).

Pseudorandom destabilisation with eyes closed (ec); subjects were tested similar as described in condition prm, however with eyes closed to prevent any visual orientation. Pseudorandom destabilisation with a sway referenced platform and visual image (srp\&s); subjects were tested similar as described in condition srp and srs combined. Pseudorandom destabilisation with eyes closed and a sway referenced platform (ec\&srp); subjects were tested similar as described in experiment SIp, however with eyes closed to prevent any visual orientation.

\section{Data analysis}

The APM during each experiment was recorded as a function of time and TPM was calculated. To evaluate the effect of training and sensory perturbation, both absolute and relative TPM changes were analysed. Absolute TPM values before and after training were compared, but also the change of TPM (before and after training) in a specific sensory condition relative to the TPM calculated in condition prm was analysed. Statistical analysis (Wilcoxon Matched-pairs Signed-ranks test, Mann Whitney $U$ test) was performed using commercially available software (PC-SPSS version 7.51 for Windows).
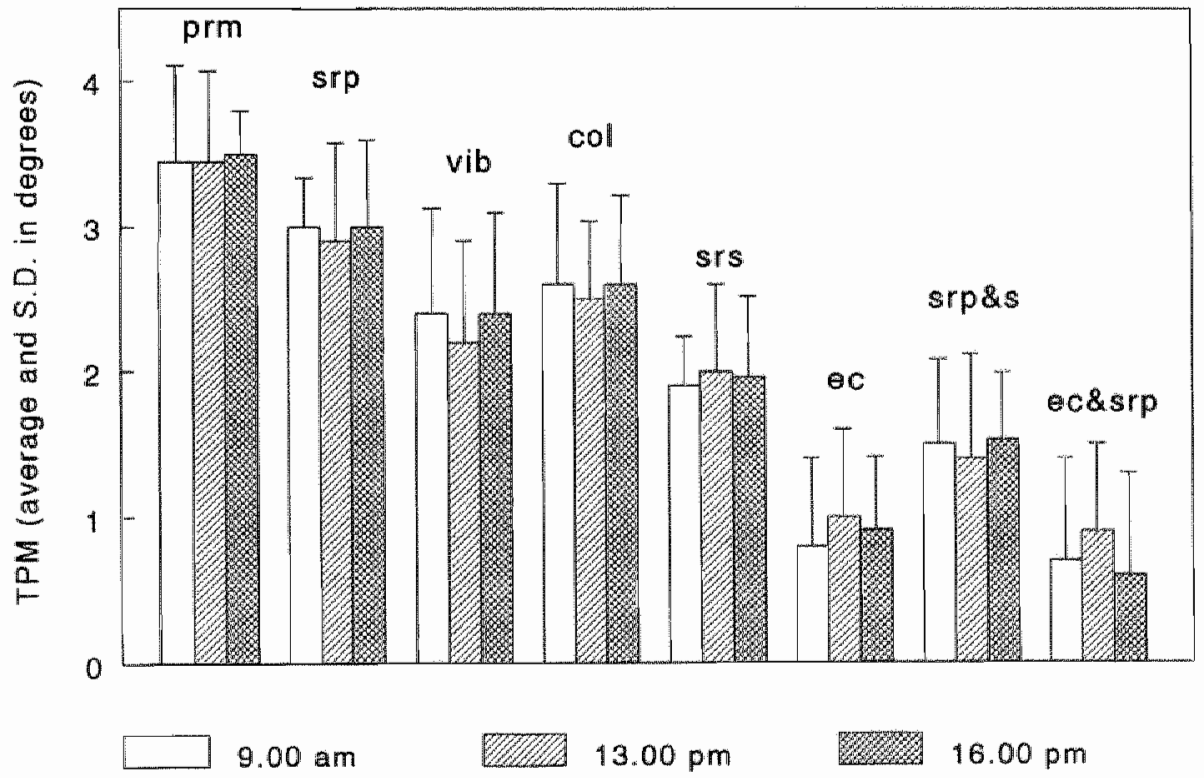

Figure 1.

TPM (mean and S.D.) in 11 healthy subjects detected at various sensory conditions at three different times on one day.

For abbreviations see text. 


\section{Results}

Experiment 1. effect of habituation to the equipment

No significant differences (paired T-tests, Wilcoxon Matched-pairs Signed rank test, $\mathrm{p}>0.05$ ) were found irrespective the sensory condition, irrespective whether the relative or absolute TPM values evaluated. The average TPM $(n=11)$ at various sensory conditions at the three different times of assessment are shown in figure 1 .

\section{Experiment 2. effect of training upon balance control}

Performance, as scored by the condition they could cope with on the training, improved (Wilcoxon Matched pairs signed rank test, $\mathrm{p}<0.0001$ ) in all 28 subjects of the training group. The condition the subjects could cope with after the first balance bar training ranged from 1 to 5 depending on the individual (average 2.4). During the last session all subjects could cope with minimal condition 4 of the balance-training program (average 6.3). Some could even cope with condition 8. In contrast none of the subjects (control and training group) reported any improvement of balance control during daily life activities.

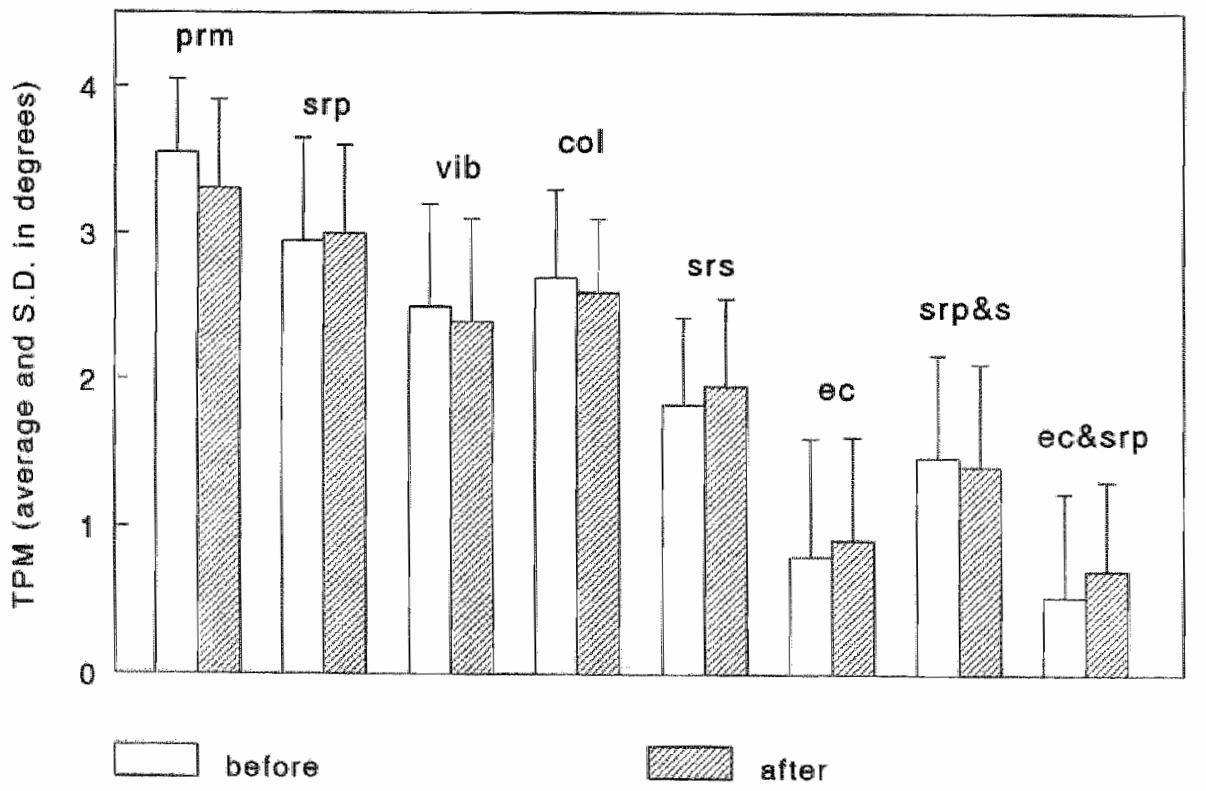

Figure 2. TPM (average and S.D) at various sensory conditions before and after balance training (training group).

For abbreviations see text. 
Figure 2 shows the absolute TPM values before and after training in the training group. The relative change of TPM (compared to TPM in condition prm) before and after training in the training group is shown in figure 3 . Figures. 4 and 5 show the results of the control group.

No significant differences (Wilcoxon Matched-pairs Signed rank test, $\mathrm{p}>0.05$ ) were found even for the trained group irrespective of the sensory condition, irrespective of the relative or absolute TPM values. The absolute TPM values and the TPM-changes of the control group and the training group were not significantly different (Mann Whitney U test, $\mathrm{p}>0.05$ ).

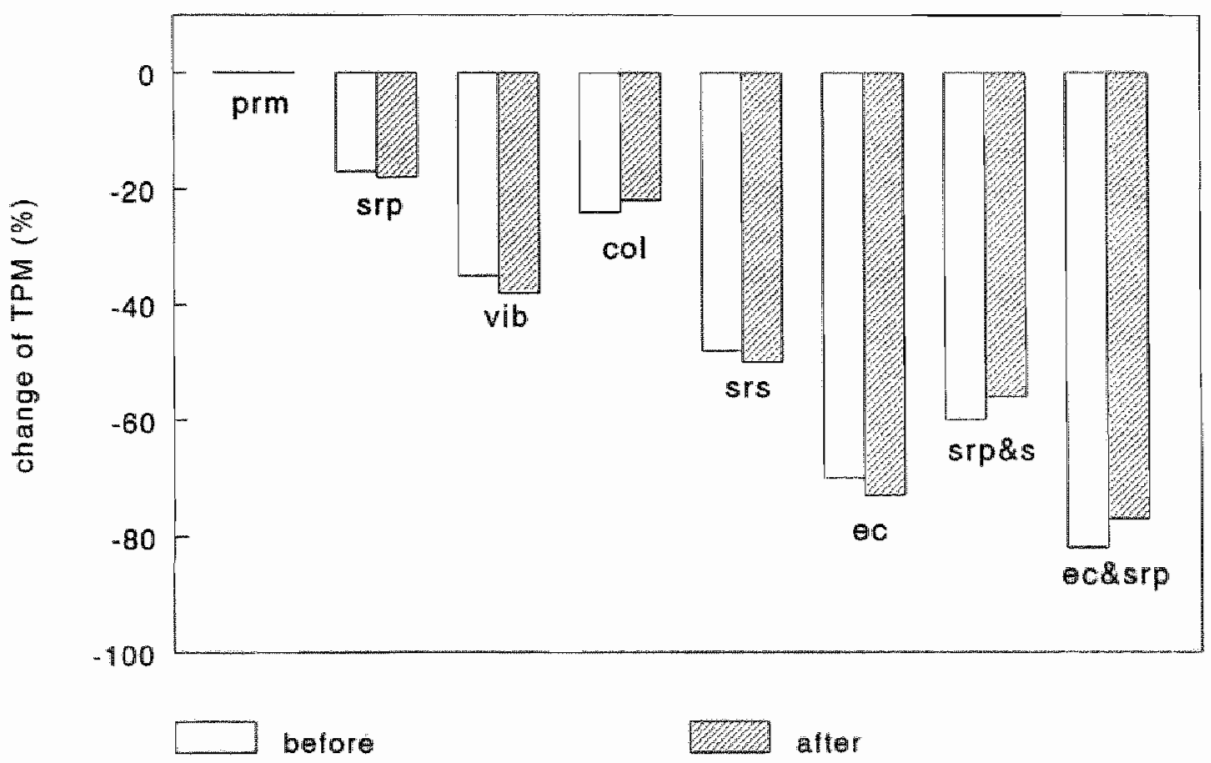

Figure 3. TPM change at various conditions relative to the TPM score in condition PRM, before and after balance training (training group). For abbreviations see text. 


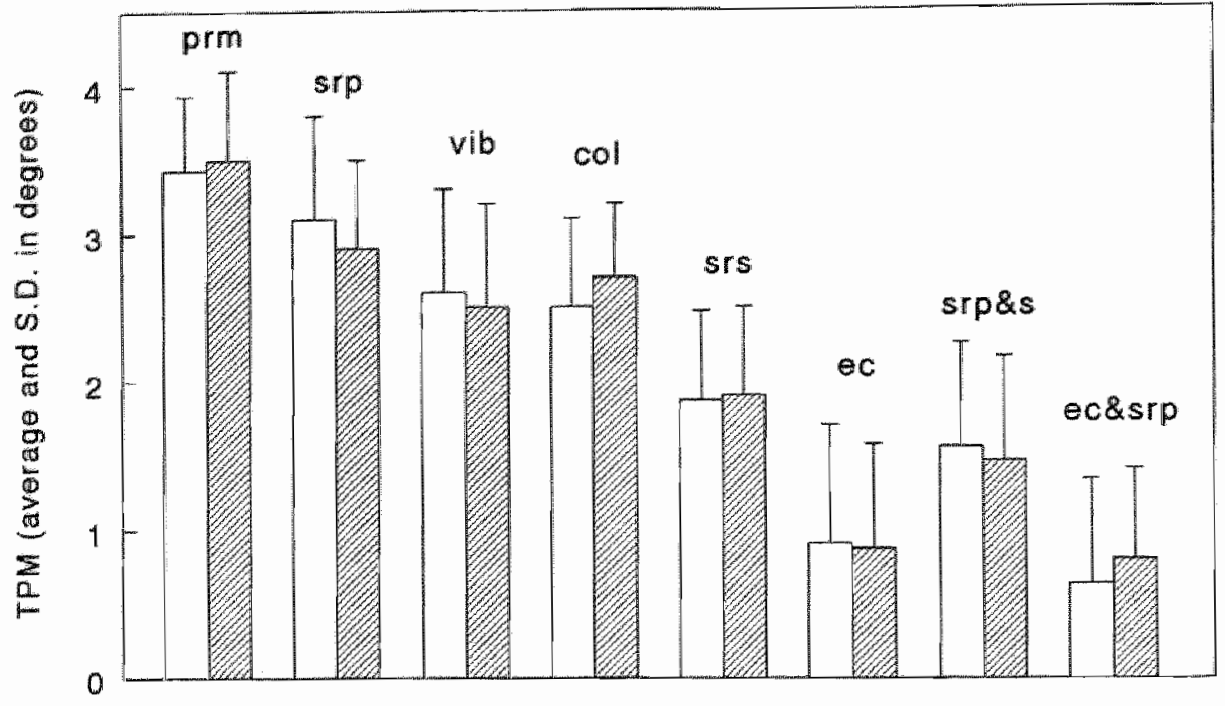

$\square$ before $\quad$ after

Figure 4. TPM (average and S.D) at various sensory conditions before and after pseudo-balance training (control group).

For abbreviations see text.

\section{Discussion}

The more or less invariable TPM values gathered during 3 consecutive measurements spread over one day, suggest that within the accuracy of the measurements no significant habituation of the subjects to the equipment or experimental conditions occurred. Apparently balance control in healthy subjects shows a remarkable stability in agreement with the literature (6).

Training on the balance bar showed a steady improvement with respect to the balancing abilities on the bar from session to session (average score increased ultimately from 2.4 to 6.3). The subjects in the 'training' and the control group reported no clear change of balance control in daily life activities despite the significant improvement of performance on the balance bar.

In a previous study (12) it was shown that the TPM score correlates well with the subjects own judgement of balance capabilities in daily life in contrast with the findings of the routine stabilometry: a high TPM is associated with an active life, a low TPM was associated with a relatively inactive. This indicated that posturography detected near the limit of stability is able to detect differences in balance control. 


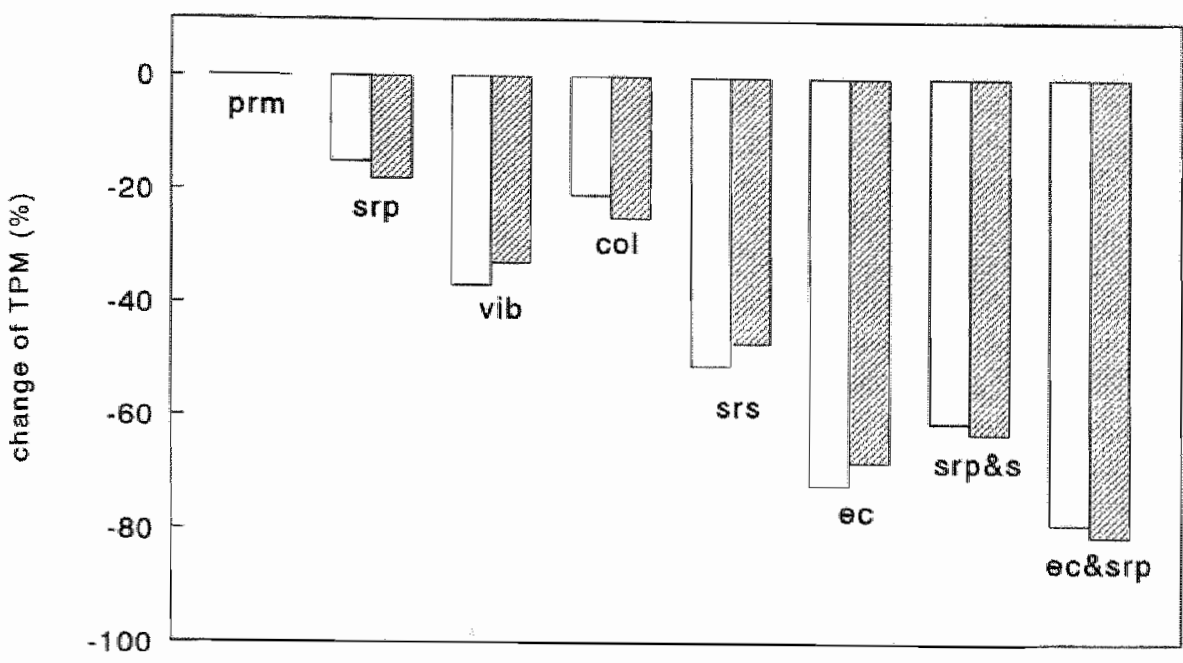

before $\quad$ alter

Figure 5. TPM change at various conditions relative to the TPM score in condition PRM, before and after pseudo-balance training (control group).

For abbreviations see text.

According to figures 2,3,4 and 5 irrespective of the sensory condition, no substantial changes in balance control had occurred despite the training. The test condition apparently does not mimic the balance bar and balance training on a bar. Also, this type of training turned out to be not effective to improve balance control and has no direct consequences for daily life activities.

Similar to stabilometry (8) and the sensory organisation test (11), dynamic posturography near the limit of stability is able to detect differences in balance control that are also reflected in daily life activities (12). But in contrast to stabilometry evaluation of posture near the limit of stability is not sensitive for specific balance training or habituation to the test equipment that have no impact on balance control in daily life activities.

Nevertheless these findings and the interpretation of the literature as presented in the introduction may give rise to another type of discussion. It is generally assumed that visual, vestibular and proprioceptive input dominate balance control, however strongly dependent on the precise stimulus context. Also, it is well established that propriocepsis is the key sensory modality to control the motor activity as the efferent part of the balance control system. Measured by dynamic posturography, the contribution of the propriocepsis towards balance control is therefore always evaluated on the sensory as well as the effector side simultaneously. Many habituation programs used for patients 
with vestibular deficits could better be characterised as 'motor training" programs; balance training which should involve training of spatial orientation is often only a small part of the program. As we did hardly measured any effect of specific balance training in this study on the test outcome, it might very well be that dynamic posturography indeed evaluates proprioceptive contributions as suggested in literature, but not because of a strong input to the sensory part (detection of the orientation relative to gravity) but more through the dominant role it must play in the efferent part of the balance control system. In short: dynamic posturography might predominantly evaluate motor control and less spatial orientation and is not that much suited to evaluate specific balance control as a reflection of spatial orientation abilities. These considerations might help in understanding the fact that acrobats have a better static balance than dancers. Dancers generally have a larger range of motion and a lot of motor training but less basic balance related activities than acrobats (8).

With respect to therapy: an improved performance on the dynamic posturography test after habituation training might primary reflect an optimisation of the motor control system. Specific training programs for optimisation of spatial orientation capabilities might however also contribute to revalidation. The latter aspect is an interesting subject for further research. 


\section{References}

1. Sloane P, Blazer D, George LK. Dizziness in a community elderly population (1983). J. Am. Geriatr. Soc. 37:101-108.

2. Shepard NT, Telian SA, Smith-Wheelock M, Raj A. Vestibular and balance rehabilitation therapy (1993). Ann. Otol. Rhinol. Laryngol. 102:198-205.

3. Hamman RG, Mekjavic I, Mallinson AI, Longridge NS. Training effects during repeated therapy sessions of balance training using visual feedback (1992). Arch. Phys. Med. Rehab. 73:738-744.

4. Harcourt JP. Posturography-applications and limitations in the management of the dizzy patient (1995). Clin. Oto-Laryngol. 20:299-302.

5. Norré ME, Forrez G, Beckers A. Vestibular compensation evaluated by rotation tests and posturography (1987). Arch. Otolaryngol. Head Neck Surg. 113:533-535.

6. Ishizaki H, Pyykko I Aalto H, Starck J. Repeatability and effect of instruction of body sway (1991). Acta Oto-Laryngol. 481: 589-592.

7. Adlerton AK, Moritz U. Does calf muscle fatique affect standing balance (1996). Scand. J. Med. Sci. Sports. 6:211-215.

8. Golomer E, Dupui P, Monod H. Sex linked differences in equilibrium reactions among adolescents performing complex sensorimotor tasks (1997). J. Physiol. Paris. 91: 49-55.

9. Hamman $\mathbb{R}$, Longridge NS, Mekjavic I, Dickinson J. Effect of age and training schedules on balance improvement exercises using visual biofeedback (1995). J. Oto-Laryngol. 24: 221-229.

10. Barona R, Zapater E, Montalt J, Armengot M, Basterra J. The effect of visual feedback exercises on balance in normal subjects (1994). Acta Oto-RhinoLaryngol. Esp. 45: 161-155.

11. Ledin T, Kronhed AC, Moller C, Moller-M, Odkvist LM, Olsson B. Effects of balance training in elderly evaluated by clinical tests and dynamic posturography (1990). J. Vestibular Res. 1: 129-138.

12. EL-Kahky AM, Kingma H, de Jong I, Dolmans $M$. Dynamic posturography in healthy subjects: evaluation of COG measuring techniques and individualisation of test conditions. Chapter 2 of this thesis.

13. EL-Kahky AM, Kingma H, de Jong I, Dolmans M. Balance control near the limit of stability at various sensory conditions in healthy subjects. Chapter 5 of this thesis. 


\section{Chapter 5}

\section{Balance control near the limit of stability at various sensory conditions in patients suffering from vertigo or balance disorders}




\section{Abstract}

Recently a new measuring system was developed to evaluate the postural system near the limit of stability where subjects are forced to perform optimally to prevent a fall. This approach resulted in a good reproducibility and allowed evaluation of the impact of the visual, vestibular and proprioceptive input to the balance control system using various perturbation techniques like sway referencing the supporting platform, sway referencing the visual surround, closing the eyes, vibration of the Achilles tendons and by chilling the foot soles. This new technique is applied in four groups of patients suffering from long lasting persistent vertigo (whiplash syndrome $(n=25)$, Ménière's disease $(n=28)$, acute $(n=28)$ or gradual peripheral function loss $(n=26)$ ) with various abnormalities detected by standard vestibular examination and a statolith function test. Balance control -on average- was maximally affected by closure of the eyes and by vibration of the Achilles tendons. The other perturbation techniques applied were less effective. The combination of vibration and a sway referencing platform was the most effective technique used to disturb the proprioceptive input. No consistent typical pattern was observed that could classify the patients with respect to the history or outcome of the standard vestibular examination: the individual data indicate that the contribution of the sensory inputs toward balance control varies considerably between patients and clearly limits the clinical relevance of this type of dynamic posturography. Balance control near the limit of stability was significantly decreased in the majority of chronic whiplash patients with vertigo or balance disorders, as well as patients suffering from Ménière's disease or patients known with a central compensated peripheral vestibular function loss. More than $89.3 \%$ of the patients could be classified with use of the balance control test, specially when the responses on the conditions with a sway referenced vision, and eyes closed (with and without the sway referenced platform) were taken into account. However, based upon their balance control no discrimination could be made between the four different patient groups.

\section{Introduction}

In most clinics quantitative evaluation of the vestibulo-ocular reflex by electronystagmography (ENG) forms the keystone for the assessment of patients with complaints of vertigo or balance disorders. However, a major problem exists (1) as many patients with such complaints are characterised by a normal outcome of this standard vestibular examination (spontaneous, gaze, saccadic, optokinetic, smooth pursuit, rotatory chair and caloric tests). Quantitative evaluation of the vestibulo-spinal reflex (VSR) is therefore considered to be of additional value to allow a more complete interpretation of the patients complaints $(2,3)$. The use of dynamic posturography to investigate the VSR system has gained more and more interest since the introduction of the computerised dynamic posturography $(4,5)$. Unlike other vestibular tests aimed at diagnosing a lesion, posturography is considered as a functional test of balance with limited diagnostic capabilities $(4,6)$. Goebel and Paige (7) reported that computerised 
dynamic posturography (CDP) enhances identification of the ballance system abnormalities in patients with and without vertigo. While the multiple tasks involved in CDP testing have been reported (8) to include test conditions sensitive to vestibular abnormalities, they found that CDP alone did not distinguish between patients with and without vertigo. They further noted that CDP provides limited help in distinguishing between central and peripheral disorders. Hamid et al (9) reported six abnormal. sensory patterns generated by the CDP. They used the results of the rotatory chair test for this classification. In contrast, Norre et al (10) reported that there is a clear disagreement in the results of the findings of the ENG and posturography in patients with peripheral vestibular disorders. The functional state of the VSR could not be predicted by the results of rotational tests, which was explained by postulating that the central compensation of peripheral function loss might be different for the VOR compared to the VSR.

The current CDP protocols are insufficient to provide the clinically desirable levels of lesion specificity $(1,11)$. Patients with partially compensated lesions often show no abnormality (12). For these patients, detection and localisation of the lesion is performed with more conventional tools, such as history, physical examination, audiometry and caloric testing. Stribly et al (13), emphasised that it is most likely necessary to apply tests with sensory conditions that are significantlly difficult to really challenge the nervous system. It seems obvious to assume that deficits of the balance control system can be unmasked only if the patient is presented with a difficult selfadjustable test stimulus to challenge his own postural system capability.

Recently we developed a measuring system that aims to evaluate the postural system near the individual limit of stability where the test subjects are forced to perform optimally to prevent a fall (14). This approach resulted in a good reproducibility that allowed an accurate comparison of the various techniques used to disturb the input of the sensory subsystems in healthy subjects (15) and is not that much influenced by the subject's motivation or habituation to the equipment. The only restriction observed in the method so far was that the individual data in healthy subjects suggests that the contribution of the sensory inputs toward balance control varies considerably between individuals which might limit the clinical relevance of dynamic posturography. In this study we evaluated this technique in patients suffering from persistent vertigo in relation to the diagnostic category and the outcome of standard vestibular examination (spontaneous, gaze, saccades, optokinetic, smooth pursuit, torsion swing, caloric test) and in 53 patients also in relation to the ocular counter rolling (OCR) upon lateroflexion as a clinical statolith test (16).

\section{Subjects}

After informed consent 30 healthy subjects ( 13 females and 17 males, age ranged from 21 to 64 years, mean age 40.2 year) and 107 patients ( 41 males and 66 females, 17-76 years old, mean age 46.2 year) with persistent complaints of instability and/or vertigo for more than nine months participated in this study. 
Only healthy subjects were included that showed a normal oto-neurological status and in which standard vestibular testing (spontaneous, gaze, saccades, optokinetic, smooth pursuit, torsion swing, calorics) revealed no abnormalities. Also body sway velocity, body sway area, as well as the associated Romberg coefficients of all subjects as revealed by computerised stabilometry was within the normal limits. The subjects had no history of neurological or musculo-skeletal problems that would affect their balance and orientation. They had no history of episodes of dizziness or underwent any ear operation and were not taking any medication at the time of testing. Only subjects were included that considered themselves as physically moderately active (incidental but no regular sport activities).

Only patients were included that showed a normal neurological status, normal outcome of the spontaneous, gaze, saccadic, optokinetic, and smooth pursuit test. In some patients abnormalities were found with the torsion swing, caloric test and/or statolith function test. The patients had no history of musculo-skeletal problems that would affect their balance and orientation. The patients did not underwent any ear operation and were not taking any medication at the time of testing. The diagnostic profile of the patients is listed in table 1 . The diagnosis of compensated acute peripheral function loss (APFL) was based upon a sudden onset of severe vertigo with a third degree spontaneous horizontal nystagmus with nausea and vomiting, as well as a caloric hyporeflexia; the symptoms decreased in all patients within three months with normalisation of all ENG findings apart from the caloric hyporeflexia, sometimes accompanied by a decreased VOR on the torsion swing test and a decreased statolith ocular reflex. Only patients with persistent vertigo and balance disorders were selected for this study. The diagnosis of gradual peripheral function loss (GPFL) was based upon a not clearly perceived, gradual onset of mild vertigo and balance disorders with a normal ENG, apart from a caloric hyporeflexia sometimes accompanied by a decreased VOR on the torsion swing test and a decreased statolith ocular reflex. The diagnosis of definite Ménière's disease was made in agreement with the guidelines of the AAOHNS 1995 classification. Seventeen (out of 28 total) Ménière's disease patients showed a caloric hypo-reflexia. All whiplash patients had persistent complaints of instability after rearend motor vehicle collisions. All of them were considered as chronic cases according to the criteria of the Quebec task force on Whiplash-Associated Disorders (17). The results of standard vestibular examination are listed in table 2 .

\begin{tabular}{|l|c|}
\hline Diagnosis \& Group & nr. of patients \\
\hline Acute peripheral function loss (APFL) & 28 \\
Gradual peripheral function loss (GPFL) & 26 \\
Ménière's disease & 28 \\
Whiplash injury & 25 \\
& -107 \\
\hline
\end{tabular}

Table 1. Diagnostic profile of the patients. 


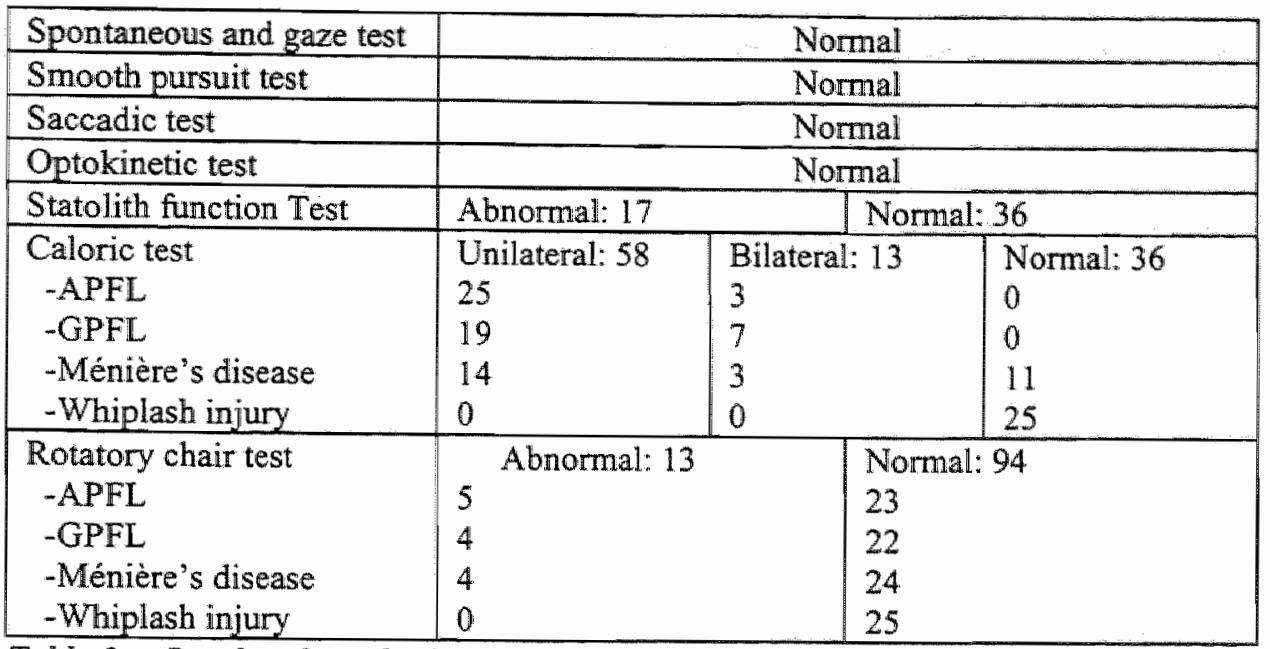

Table 2. Results of standard vestibular examination of the patients.

The criteria for normality used were as follows:

Spontaneous test:

Gaze test:

Smooth pursuit:

Optokinetic test:

Torsion swing test:

Caloric test: slow component velocity $(\mathrm{SCV})<5 \mathrm{dg} / \mathrm{s}$

no nystagmus

position gain $>87 \%$, phase $(-3$ to $+3 \mathrm{dg})$

minimal $\mathrm{SCV}>23 \mathrm{dg} / \mathrm{s}$ at $40 \mathrm{dg} / \mathrm{s}$ stimulation

gain $>.30$, phase $(-10$ to $10 \mathrm{dg})$

cold irrigations SCV $>8 \mathrm{dg} / \mathrm{s}$, warm irrigations $>10 \mathrm{dg} / \mathrm{s}$

unilateral weakness $<20 \%$, nystagmus preponderance $<$ $25 \%$.

In 53 patients ocular counter rolling (OCR) upon 40 degrees lateroflexion was measured as a clinical statolith function test using video oculography $(16,23)$. Seventeen patients showed a reduced $O C R$ upon lateroflexion $(<=4$ degrees) of which 4 patients out of 11 with a history of acute peripheral function loss, 4 patients out of 13 with a history of gradual peripheral function loss, whereas 5 patients out of 9 with Ménière's disease, and 4 out of 20 with a whiplash injury.

\section{Methods}

The experimental set-up composed of a high resolution $(<0.1 \mathrm{~cm})$ home built real time infrared (IR) video body movement tracking system, a dynamic force plate (T-Post, Jaeger-Toennies GmbH, Wuerzburg, Germany) provided with a device for cooling the foot soles (Instrumental Service Maastricht University (ISMU, The Netherlands)], a duall Achilles" tendon vibrator (ISMU, The Netherlands), a dynamic slide projection system (ISMU, The Netherlands and Jaeger-Toennies GmbH, Wuerzburg, Germany, patent PT1265390) and a modified Nystagliner system (Jaeger-Toennies GmbH, 
Wuerzburg, Germany) for data-acquisition (set at $50 \mathrm{~Hz}$ per channel) and stimulus control (platform, slide projection, vibrators). The $50 \mathrm{~Hz}$ IR-video tracker system consisted of a video camera focused on the left side of the subjects body. The camera inaged the position of five active IR markers fixed to the ankle, knee, hip and shoulder joints and near the axis of head nodding. The IR marker positions (,$y$ in the anteriorposterior plane) were detected in the video frame, grabbed by a personal computer and subsequently transferred to the data-acquisition PC (Nystagliner). A biomechanical model (14) was used to calculate the position and velocity of the centre of gravity of the body (COG) in the anterior-posterior plane based upon the marker positions detected. Body sway velocity was calculated by differentiating ( 3 points) the digitised position signal. The data-acquisition computer also controlled the dynamic platform that could rotate up to 9 degrees maximum with a maximum angular velocity of 50 degrees/second and maximum angular acceleration of 1400 degrees $/ \mathrm{sec}^{2}$ (maximum delay $18 \mathrm{~ms}$ ). The rotation axis of the platform coincided with the axis through the ankle joints. The dynamic slide projector imaged (slightly out of focus for optimal visual impact) a random dot pattern on a transview screen (width $3.20 \mathrm{~m}$., height 2.40 $\mathrm{m}$.), $0,50 \mathrm{~m}$. in front of the subject. Both slide position and orientation, and the focal length of the projection lens was controlled by four computer (Nystagliner) driven synchro motors (maximum delay $2 \mathrm{~ms}$ ). As a consequence the image could rotate, translate and zoom in, zoom out to 'counteract' any body or head motion relative to the projection screen, or to mimic any motion of the visual reference frame. Besides the event and time control of the test procedures, the software was able to use 8 signals as an input to produce 8 output signals to control platform rotation or slide position according to a user defined transfer function. The vibration was applied through home built vibrators attached to the right and left Achilles' tendon by an elastic strip. The computer controlled vibrator's DC-motor (Simprop Electronic) housed in a cylinder allowing a certain degree of movement to govern the amplitude of the vibration with a maxinum frequency of $250 \mathrm{~Hz}(+/-10 \mathrm{~Hz})$ and a maximum amplitude of $0.2 \mathrm{~cm}$. The cold supporting surface consisted of a metal box filled with ice-water (about $1-3{ }^{\circ} \mathrm{C}$ ), specially designed to be placed on the dynamic platform without affecting its movement (ISMU, The Netherlands). It must be marked that the position of the rotation exis was affected by this procedure, but was not taken into considerations with respect to the calculations of the performance.

\section{Experiments}

\section{General aspects}

In all experiments subjects were asked to stand as stable as possible on the dynamic platform, bare feet, arms folded at the waist, looking straight ahead, facing the slide projection screen without leaning forward or backward during each test period of 20 seconds. Temporal vision was blocked by mounting special goggles to the subject. Additionally, all subjects wore a head phone supplied with soft, not distracting music to prevent spatial orientation on auditory cues. COG position and velocity was continuously measured by means of the video system. The basic platform movement 
$(14,15)$ consisted out of 3 mutual harmonically unrelated frequencies $(0.16,0.42,1.15 \mathrm{~Hz})$ with an initial sum of all three amplitudes of 2 degrees. During the duration of the experiment the Amplitude of the platform movement (APM) increased automatically in time with 0.004 degree per $20 \mathrm{~ms}(0.2 \mathrm{dg} / \mathrm{s})$ as long as COG velocity was below $5 \mathrm{~cm} / \mathrm{sec}$. APM was fixed when the subject could maintain the COG velocity within the range from 5 $-8 \mathrm{~cm} / \mathrm{sec}$.APM automatically decreased with $0.004 \mathrm{dg}$ per $20 \mathrm{~ms}(0.2 \mathrm{dg} / \mathrm{s})$ when COG vellocity exceeded $8 \mathrm{~cm} / \mathrm{sec}$.APM also decreased whenever $50 \%(3.125 \mathrm{dg})$ of the maximum angle ( $6.25 \mathrm{dg}$ ) of forward or backward leaning ("the cone of stability") was reached irrespective the actual body sway velocity, to prevent the subject from falling. Balance control was expressed in terms of the tolerated platform movement 'TPM' quantified as the average APM tolerated during the $20 \mathrm{sec}$.test duration. With an initial APM of 2 degrees and a maximum increase of $0.2 \mathrm{dg} / \mathrm{s}$ this resulted in a maximum APM of $6 \mathrm{dg}$ after $20 \mathrm{sec}$.and a maximum TPM of $4 \mathrm{dg}$. The effect of manipulation of the sensory inputs upon the TPM was studied. In all subjects eight different sensory conditions were evaluated.

Pseudorandom movement ( $p r m$ ); the subjects base of support was destabilised by the pseudo random rotation of the platform alone during normal vision (projection of a random dot pattern). Pseudorandom destabilisation with a sway referenced platform (srp): subjects were tested similar as described condition prm, however -besides the basic pseudorandom movement- the platform angle moved also according to the body sway angle relative to the vertical with a positive feed back gain of 0.5 . Pseudorandom destabilisation with vibrators (vib); subjects were tested similar as described in condition prm with activated vibrators attached to the right and left Achilles' tendon. Pseudorandom destabilisation with cooled foot soles (col); subjects were tested similar as described in condition prm, however the supporting surface was equipped with a metal box filled with ice-water. Prior to this test, the subjects were seated on a chair, the feet placed on the cooling-device (mounted on the rotating platform) for 5 minutes. After the feet were pre-chilled, the subjects were asked to stand up and the actual measurement took place immediately thereafter. $P_{S e u d o r a n d o m}$ destabilisation with a sway referenced visual image (srs); subjects were tested similar as described in condition prm, however the projected image of the slide moved according to the posterior-anterior sway of the COG (positive feed back gain of 1.0 ). Pseudorandom stabilisation with eyes closed (ec): subjects were tested similar as described in condition prm, however with eyes closed to prevent any visual orientation. Pseudorandom destabilisation with a sway referenced platform and visual image (srp\&s); subjects were tested similar as described in condition srp and sis combined. Pseudorandom destabilisation with eyes closed and a sway referenced platform (ec\&srp); subjects were tested similar as described in experiment srp, however with eyes closed to prevent any visual orientation.

\section{Data-analysis}

The APM during each experiment was recorded as a function of time and TPM was calculated. Statistical analysis (Mann-Whitney U-test and Wilcoxon Matched-pairs 
Signed-Rank test, Discriminant analysis, Hierarchical and Kruskal Wallis Cluster Analysis) was performed using commercially available software (PC-SPSS version 7.52 for Windows).

\section{Results}

\section{Geweral aspects}

Figure 1 shows an example of the APM as a function of time in a patient with Ménière's disease when the subjects base of support was destabilised by the pseudo random rotation of the platform alone during normal vision (prm), the effect of perturbation upon APM by disturbing the propriocepsis (srp, vib, col), vision (srs, ec) and the effect of simultaneous perturbation of propriocepsis and vision (srs\&s, ec\&srp) in a patient suffering from Ménière's disease. Figure 2 summarises the results obtained in this study. The mean TPM at eight different sensory conditions per diagnostic group (healthy subjects, patients suffering from persistent vertigo and balance disorders after an acute or gradual arisen peripheral vestibular function loss, Ménière disease and whiplash patients) are shown.

Tables $3 a, b, c, d$,e show the mean and range of all groups at the eight sensory conditions.

Comparison of the conditions within the diagnostic groups

Differences of TPM at various sensory conditions per diagnostic patient group were evaluated by Wilcoxon Matched-pairs Signed Rank tests. For all groups, TPM in condition srp, vib, col, srs, ec, srp\&s and ec\&srp was significantly smaller than in condition prm $(\mathrm{p}<0.01)$. 

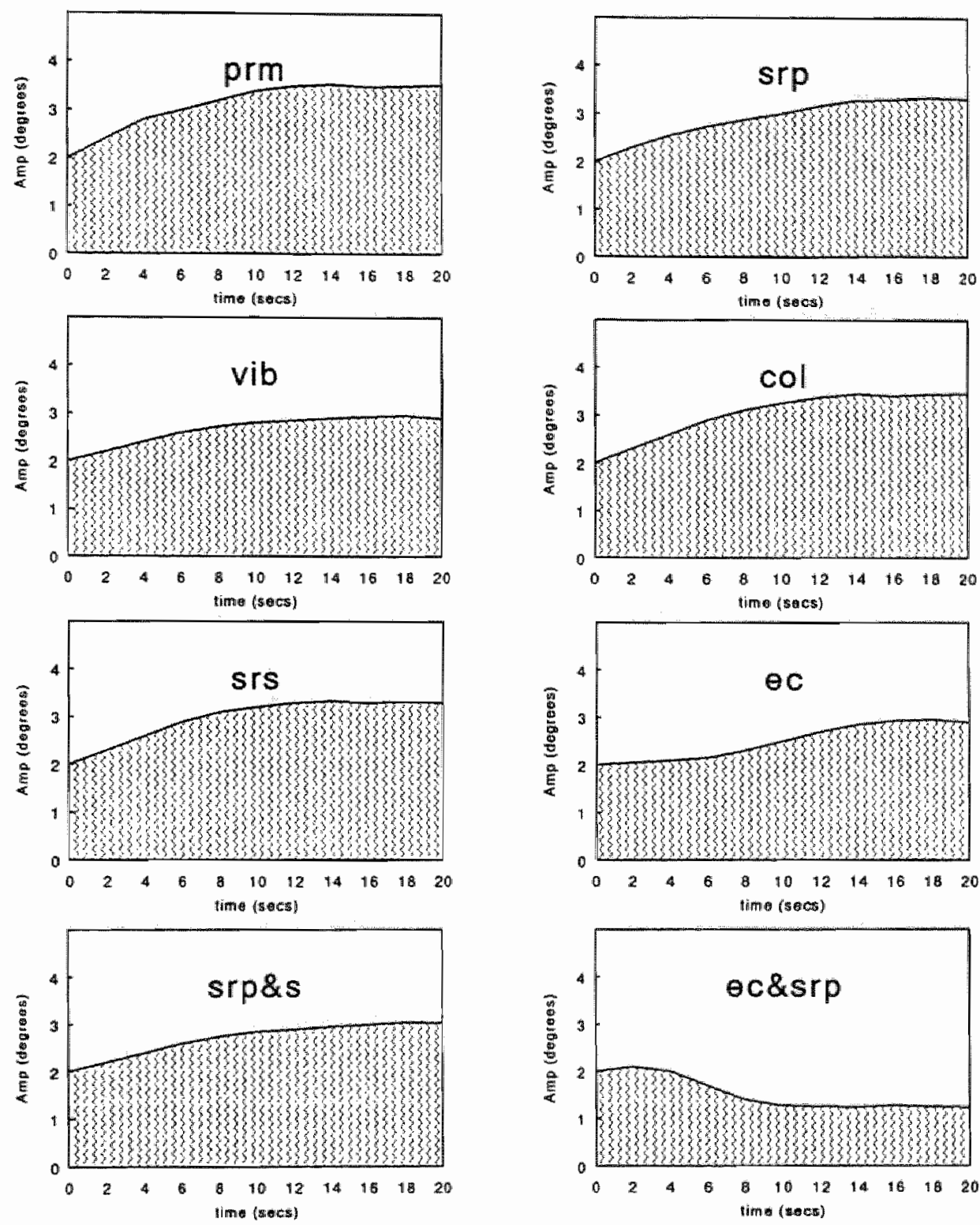

Figure 1. APM as a function of time in a patient with Ménière's disease. prm: psendo random movement; srp: sway referenced platform; wib: vibrators attached to the Achilles tendons; col: cooled foot soles; srs: sway referenced surround; ec: eyes closed; srp\&s: sway referenced platform and surround: ec\&srp: eyes closed and sway referenced platform. 


\section{Comparison of the conditions ec and srs}

Compared to condition prm $_{*}$ ec reduced TPM more than srs in healthy subjects, the "gradual peripheral function loss" group and "Menière" group $(p<0.033)$. In the "whiplash" group srs was as effective as ec (srs was relatively more effective in whiplash patients than in the other diagnostic groups).

Comparison of the conditions srp, vib and col

Compared to condition prm, vib reduced TPM more than col or srp in all but the Ménière's disease patients $(p<0.007)$. In healthy subjects srp reduced TPM more than $\mathrm{col}(\mathrm{p}<0.003)$ but in all patients srp was equally effective as col $(p<0.05)$.

\section{Comparison of the conditions srp\&s and ec\&srp}

Compared to condition prm, ec\&srp reduced TPM more than srp\&s in all but the whiplash patients $(\mathrm{p}<0.013)$. In whiplash patients both conditions reduced TPM equally effective ( $p<0.05$, srp\&s was relatively more effective in whiplash patients than in the other diagnostic groups).

\section{Comparison between the diagnostic groups}

Differences of TPM at various sensory conditions per diagnostic patient group were evaluated by Mann Whitney U-tests. For all conditions the average TPM between healthy subjects and any patient group was significantly different $(p<0.002)$. TPM differs significantly between Whiplash patients and patients with Ménière disease for the conditions srs (Mann Whitney U-test, $p<0.034$ ) and srp\&s (Mann Whitney U-test, $p<0.008$ ). However no other significant differences were observed between the four diagnostic patient groups, irrespective the sensory condition ( $p>0.05$ ).

\section{Analysis of predictors for group memberships}

With use of discriminant analysis we explored which sensory conditions contributed most to a possible discrimination between healthy subjects and the four patient groups. Conditions ec, ec\&srp and srs appeared to contribute maximum with however only $47.4 \%$ of the cases correctly classified. Approaching the problem from the other side: a hierarchical cluster analysis with no pre-assumptions about the number of clusters (all 8 conditions included as parameters), or a Kruskal Wallis cluster analysis with the preassumption that 5 clusters should be distinguished, resulted in even worse discrimination. Based upon this result, the graphs displayed in figure 2, the results of the statistics using the Mann Whitney U-tests, we concluded that the major problem seems to arise from the fact that the performance of the four patient groups is very similar and only differs from the healthy subject group. We therefore re-analysed the data by discriminant analysis, now defining three groups: 1 . healthy subjects, 2. vestibular patients (acute or gradual peripheral function loss, Ménière's disease) and 3. whiplash patients. Again conditions ec, ec\&srp and srs appeared to contribute maximum; however only $73.2 \%$ of the cases were correctly classified. 


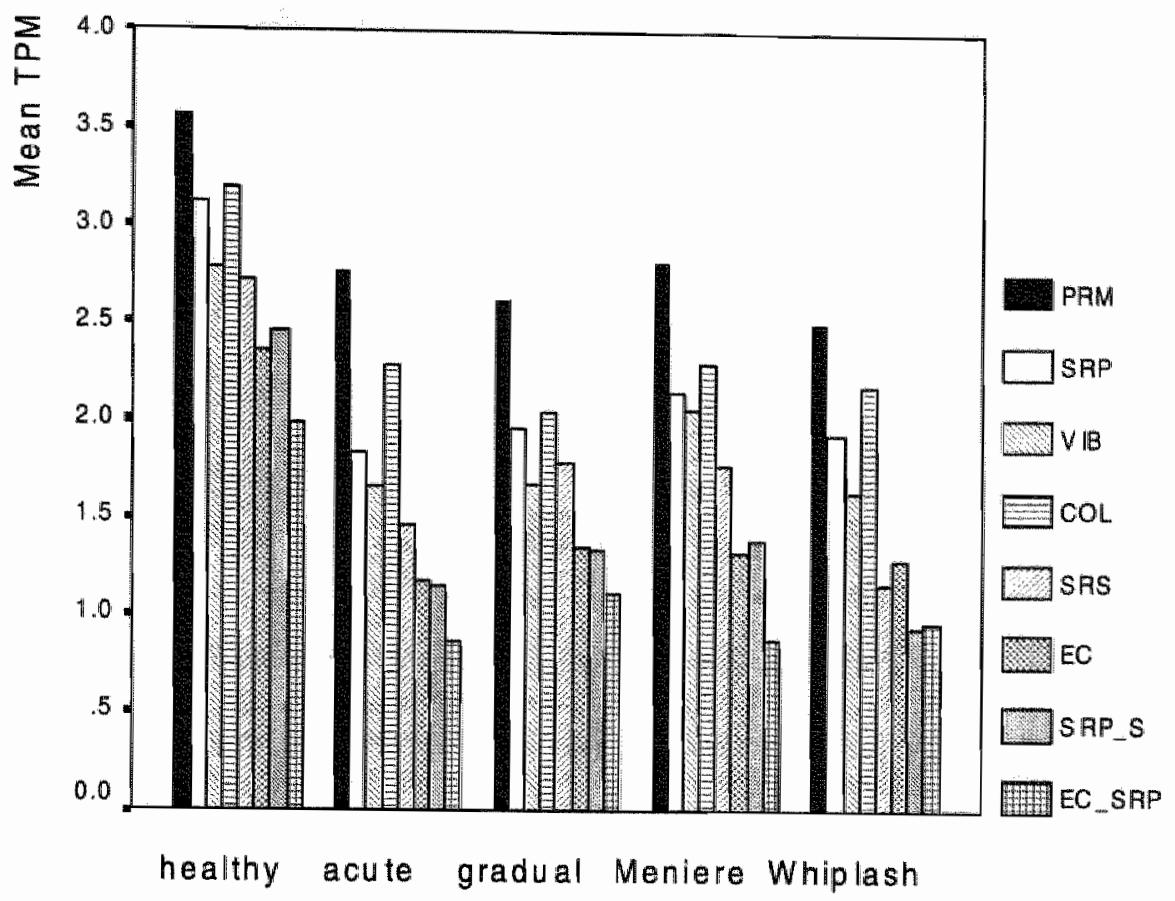

Figure 2. $\quad$ Mean TPM at eight different sensory conditions per diagnostic group (healthy subjects (abbreviation: healthy), patients suffering from persistent vertigo and balance disorders after an acute (abbreviation: acute) or gradual (abbreviation: gradual) arisen peripheral vestibular function loss, Ménière's disease (abbreviation: Meniere) and whiplash patients (abbreviation: Whiplash)). For all other abbreviations see text.

Performing a Kruskal Wallis cluster analysis by pre-definition of three possible clusters (three diagnostic groups) the five diagnostic groups studied here could be classified into healthy (cluster 2), vestibular or whiplash (cluster 1 or 3 ). The result is displayed in figure 3. As this result was still disappointing a renewed analysis was performed, now defining only two groups: healthy subjects and patients. Conditions ec, ec\&srp and srs appeared to contribute maximum again, now with $90.6 \%$ of all cases correctly classified ( $93.5 \%$ of the healthy subjects, $89.3 \%$ of the patients). Performing the cluster analysis now by pre-definition of two possible clusters (three diagnostic groups) the five diagnostic groups studied here could be classified into healthy (cluster 1) or patient (cluster 2). The result per group is displayed in figure 4. Figure 5 shows the result if the four patient groups are summed and no discrimination is made anymore to sub groups. 


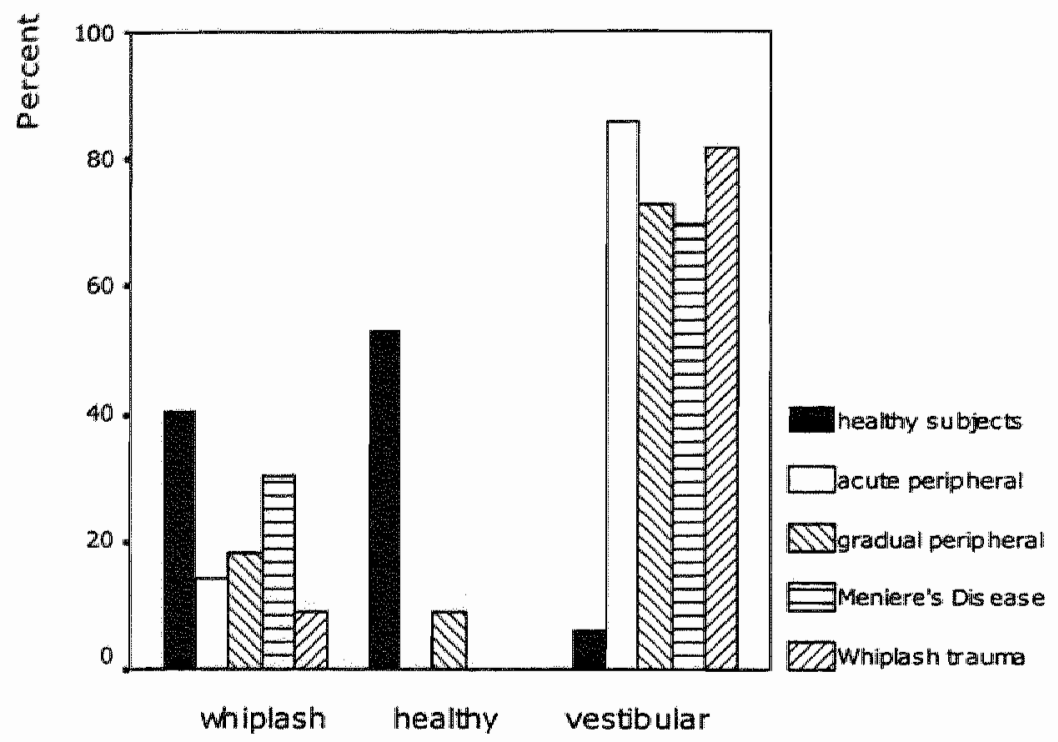

Figure 3. Subjects classification result of the Kruskal Wallis cluster analysis based upon the test outcome of the conditions ec,ec\&srp and srs, by pre-definition of three possible clusters: healthy subjects (abbreviaton: healthy), patients with a vestibular dysfunction (abbreviation: vestibular) and whiplash patients (abbreviation: whiplash).

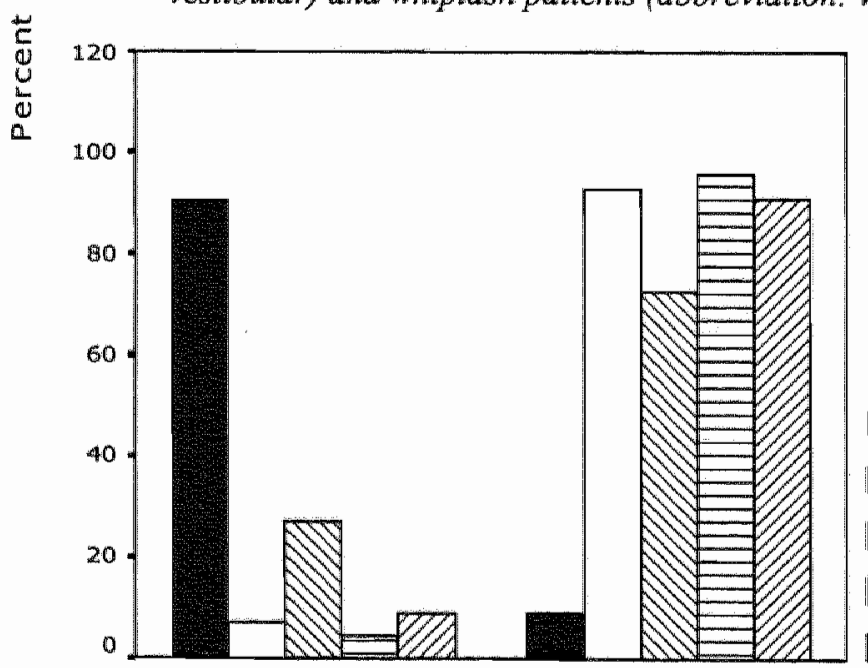

heatthy subjects

Jacute pepipheral

Algradual peripheral

EMeniere's Dis ease

QWhiplash traumb

normal test

abnormall test

Figure 4. Subjects classification result of the Kruskal Wallis cluster analysis based upon the test outcome of the conditions ec, ecdsrp and srs, by pre-definition of two possible clusters: healthy subjects (abbreviaton: healthy) and all patient categories clustered (abbreviation: patient). 


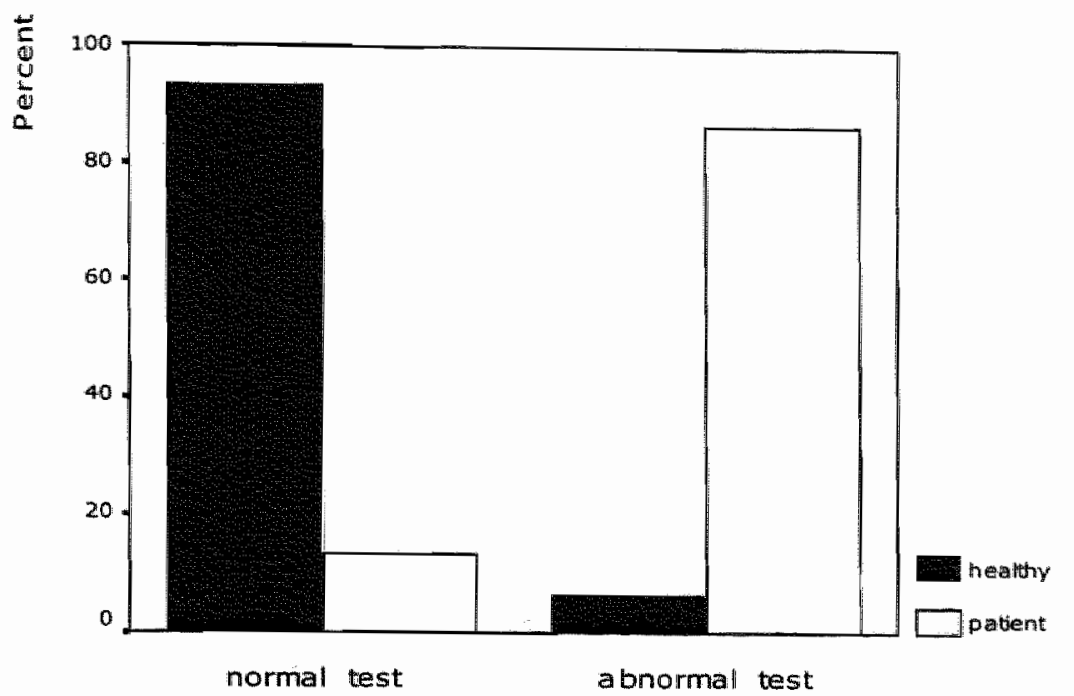

Figure 5. Subjects classification result of the Kruskal Wallis cluster analysis based upon the test outcome of the conditions ec, ec\&srp and srs, by pre-definition of two possible clusters healthy subjects (abbreviation: healthy) and all patients categories (abbreviation: patient). The result of the four patient groups is summed; no discrimination is made into sub groups.

Correlation's between findings of posturography and standard vestibular tests In this study patients with a history of an acute or gradual peripheral function loss were classified -among other criteria- upon a caloric hypo-reflexia. As all these patients showed a reduced performance upon the posturography test, a $100 \%$ correlation was found. Performance was maximally reduced in case of a bilateral caloric hyporeflexia (not areflexia) but not significantly different from the patients with a unilateral caloric hyporeflexia. Seventeen out of 28 Ménière's disease patients showed a calloric hyporeflexia; there was no significant difference in balance control regardless the sensory condition $(\mathrm{p}>0.05)$. No abnomalities with the caloric test were observed in the whiplash patients that nevertheless also showed a reduced balance control not significantly different from the patients with a peripheral function loss. Figure 6 show TPM scores in Ménière's disease patients and patients with a history of a (gradual or acute) peripheral function loss separated by the outcome of the torsion swing test (abnormal $n=13$, normal $n=69$ ). No significant differences were found, irrespective the sensory condition ( $p>0.05$ ). Figure 7 show TPM scores in Ménière's disease patients, whiplash patients and patients with a history of a (gradual or acute) peripheral function loss separated by the outcome of the statolith test (abnormal $n=17$, normal $n=36$ ). No significant differences were found, irrespective the sensory condition $(p>0.05)$. 


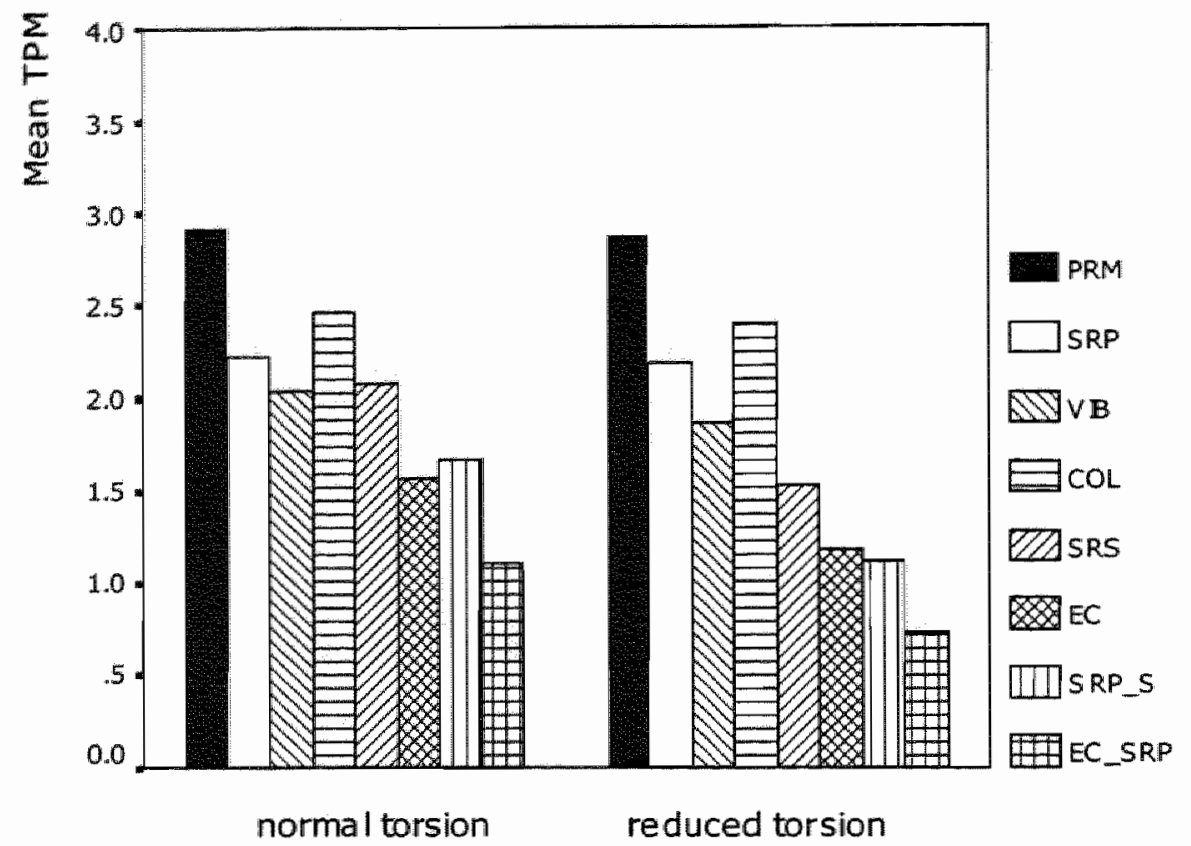

Figure 6. TPM scores in Ménière's disease patients and patients with a history of a (gradual or acute) peripheral function loss separated by the outcome of the torsion swing test (abnormal $n=13$, normal $n-69$ ). No significant differences were found, irrespective the sensory condition $(p>0.05)$. For abbreviations see text.

\section{Discussion}

Discrimination between patients and healthy subjects

The influence of peripheral vestibular dysfunction upon the vestibulo-spinal reflex has been investigated by many authors $(18,19)$. Norre and Forrez (18) reported that a normal posturography test outcome was found in $32.5 \%$ of the patients with peripheral vestibular disorders. In another report by Norre (20) normal results were found in $25.3 \%$ in a group of patients with Ménière's disease. Morrison et al (21) found no significant difference between normals and 17 patients with Ménière"s disease using custom built posturography. Hamid et al (9), using the sensory organisation tests (SOT, often considered as the standard dynamic posturography test), reported that the sensitivity of the abnormal sensory pattern provided by the SOT is near $90 \%$. In contrast Barin et al (12) reported that the SOT test outcome in patients with unilateral peripheral vestibular loss are within normal limits. They reported that significant differences were found only with different head orientation (head extension or tilt). 


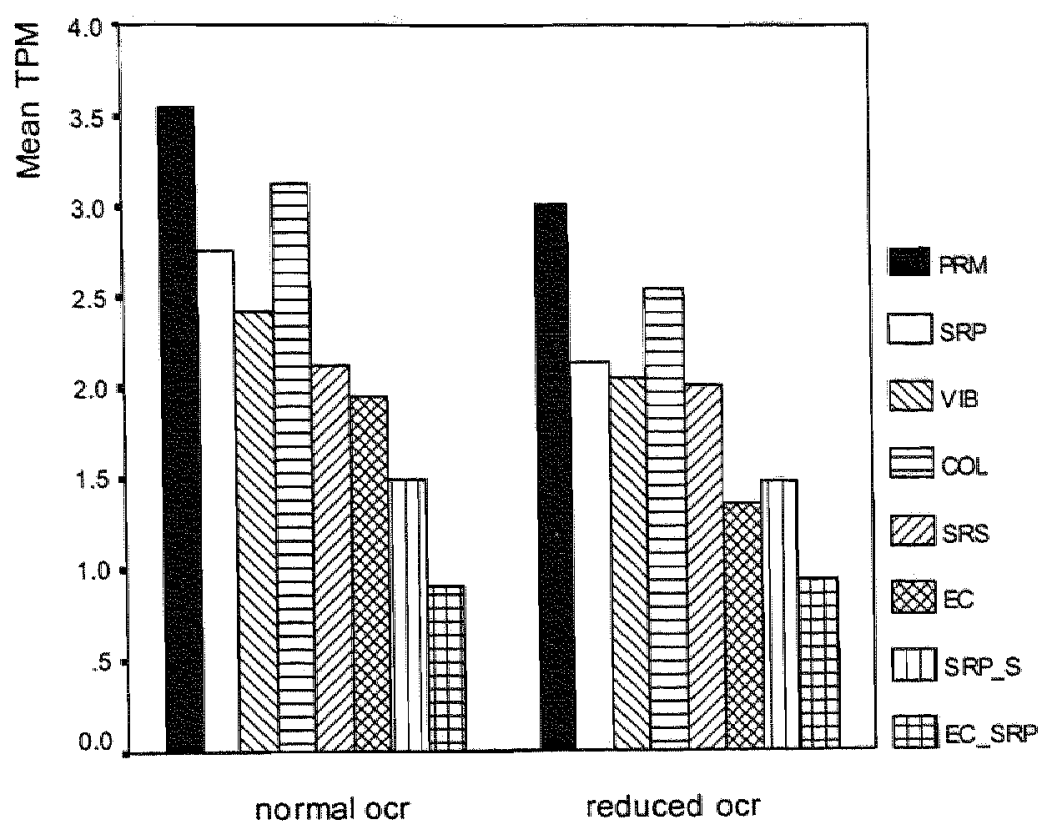

Figure 7. TPM scores in Ménière's disease patients, Whiplash patients and patients with a history of a (gradual or acute) peripheral function loss separated by the outcome of the statolite tests (abnormal $n=17$, normal $n=36$ ). No significant differences were found, in respective the sensory condition $(p=0.05)$. ocr: ocular counter rolling upon latero-flexion. For all other abbreviations see texi.

In 338 patients with peripheral vestibular disorders, it was reported (11) that the sensitivity of the sensory organisation test (SOT) was only $40 \%$. It is extremely complicated to understand the background of the discrepancies in literature. First, it must be stressed that postural performance is context dependent: any change in the sensory conditions may activate different control systems and efference copies and lead to confusing results in the same subject. Also, major abnormalities in balance control revealed by stabilometry or dynamic posturography seem to be only present directly after the onset of an acute vestibular function loss or vertigo attack, and many authors indicate that the findings tend to normalise within several weeks after the onset thanks to central compensation and vestibular habituation training. Strikingly, none of our patients did suffer from a recent acute vestibular function loss, but nevertheless we found a significant decreased balance control, in all sensory conditions, compared to healthy subjects. This suggests that quantification of balance control near the limit of stability is more sensitive than the standard procedures used by stabilometry or the sensory organisation test. However, the result of the statistics involved (Mann Whitney U-tests) refer to an evaluation of the population values of TMP and do not indicate 
whether proper discrimination can be made on an individual base. In a pilot study we observed that the test-retest variability in patients is not more than that observed in healthy subjects $(<14.5 \%)(14,15)$, which indicates that the wide range of TPM scores depicted in table 3 is due to inter-individual variability. These individual data therefore indicate a complication: the sensitivity for the various perturbation techniques seems to vary widely between the subjects, as is depicted in table 3 . This suggests that the contribution of the sensory inputs toward balance control differs considerably per individual and may be simply due to differences in the vestibular function related to the specific pathology, but also due to differences in motor leaming strategies in relation to daily life requirements. This aspect limits the discrimination between patients and healthy subjects, and limits the localisation of deficits of the three sensory subsystems in patients based upon measurements of balance control. In agreement with this, no clear cut, significant differences are observed between the four diagnostic groups, apart from some minor findings. In healthy subjects and all other patients groups but whiplash patients, balance control is more effected by exclusion of visual cues (ec) than by misleading visual cues (srs). Whiplash patients are more sensitive to misleading visual cues: their performance is as bad with srs as with ec. Roos (22) measured increased postural sway with stabilometry in whiplash patients with different head position compared to healthy subjects; the results were interpreted assuming that the disturbed sensory information leads to a shift in the relative weight of the sensory information in favour of vision. This hypothesis could also explain our data that indicate that whiplash patients tend to rely more on (wrong) visual cues (srs and srp\&s) than all other subjects studied. However, a good explanation for this observed functional change related to the trauma still fails.

So far only isolated sensory conditions were used to search for differences between the four diagnostic groups and the healthy subjects. To assess the power of a combination of outcomes, discriminant analyses and cluster analyses were performed. The discriminant analysis indicated that the "most difficult" conditions ec, srs and ec\&srp are also the most relevant: with the outcome of these 3 conditions in $90.6 \%$ of the cases. patients and healthy subjects could be distinguished from each other (figure 5). This indicates that even on an individual base classification can be achieved; this "predictive" aspect needs verification in a second study using the selection parameters calculated in this study. Unfortunately, discrimination is not precise, no distinction can be made into a patient category (figure 4) and no specific pattern could be observed that could be correlated to a patient category (figure 2).

\section{Impact of manipulation of sensory input on balance control: most effective techniques}

In patients, similar to what was found in a previous study dealing with balance control near the limit of stability in healthy subjects (15) the ec condition appeared to be the most effective visual condition to affect balance control, whereas the vib condition appeared to be the most effective proprioceptive condition to reduce the TPM score. Nevertheless, additional incorporation of the srs and ec\&srp condition in the test battery appeared to be essential to obtain an optimal discrimination between patients and healthy subjects. 


\begin{tabular}{|l|l|l|l|}
\hline & Minimum & Maximum & Mean \\
\hline prm & 1.68 & 3.98 & 3.5573 \\
srp & 1.37 & 3.94 & 3.0827 \\
vib & 1.42 & 3.78 & 2.7826 \\
col & 1.02 & 3.98 & 3.1890 \\
srs & 0.40 & 3.72 & 2.7007 \\
ec & 0.76 & 3.30 & 2.3391 \\
srp \& s & 0.00 & 3.87 & 2.4376 \\
ec \& srp & 0.41 & 3.67 & 1.9805 \\
\hline
\end{tabular}

Table $3 a$. Healthy subjects.

\begin{tabular}{|l|l|l|l|}
\hline & Minimum & Maximum & Mean \\
\hline prm & 1.34 & 3.82 & 2.7759 \\
srp & 1.09 & 3.22 & 1.8421 \\
vib & 0.96 & 2.74 & 1.6625 \\
col & 1.22 & 3.51 & 2.2800 \\
srs & 0.56 & 2.66 & 1.4659 \\
ec & 0.52 & 1.86 & 1.1771 \\
srp \& s & 0.44 & 2.63 & 1.1505 \\
ec \& srp & 0.51 & 1.86 & 0.8740 \\
\hline
\end{tabular}

Table $3 b$. History with acute pripheral function loss.

\begin{tabular}{|l|l|l|l|}
\hline & Minimum & Maximum & Mean \\
\hline prm & 1.60 & 3.71 & 2.6237 \\
srp & 0.85 & 3.66 & 1.9648 \\
vib & 0.69 & 3.15 & 1.6770 \\
col & 0.94 & 3.09 & 2.0482 \\
srs & 0.69 & 3.35 & 1.7919 \\
ec & 0.61 & 2.54 & 1.3542 \\
srp \& s & 0.55 & 2.81 & 1.3469 \\
ec \& srp & 0.63 & 2.46 & 1.1232 \\
\hline
\end{tabular}

Table 3c. History with gradual onset of peripheral function loss.

\begin{tabular}{|l|l|l|l|}
\hline & Minimum & Maximum & Mean \\
\hline prm & 1.20 & 3.70 & 2.7433 \\
srp & 0.67 & 3.62 & 2.0736 \\
vib & 0.53 & 3.01 & 1.9853 \\
col & 0.73 & 3.30 & 2.2956 \\
srs & 0.69 & 3.39 & 1.7851 \\
ec & 0.53 & 2.37 & 1.3277 \\
srp \& s & 0.68 & 3.06 & 1.3381 \\
ec \& srp & 0.51 & 1.40 & 0.8512 \\
\hline
\end{tabular}

Table 3d. Ménière's disease. 


\begin{tabular}{|l|l|l|l|}
\hline & Minimum & Maximum & Mean \\
\hline prm & 1.20 & 3.74 & 2.4013 \\
srp & 0.61 & 3.10 & 1.8294 \\
vib & 0.72 & 2.54 & 1.6238 \\
col & 1.02 & 3.30 & 2.0845 \\
srs & 0.57 & 1.77 & 1.1717 \\
ec & 0.69 & 1.74 & 1.2381 \\
srp \& s & 0.55 & 1.58 & 0.9165 \\
ec \& srp & 0.59 & 1.74 & 0.9358 \\
\hline
\end{tabular}

Table 3e. Whiplash trauma.

Impact of manipulation of sensory input on balance control: most effective techniques In patients, similar to what was found in a previous study dealing with balance control near the limit of stability in healthy subjects (15) the ec condition appeared to be the most effective visual condition to affect balance control, whereas the vib condition appeared to be the most effective proprioceptive condition to reduce the TPM score. Nevertheless, additional incorporation of the srs and ec\&srp condition in the test battery appeared to be essential to obtain an optimal discrimination between patients and healthy subjects.

\section{Relation outcome of standard vestibular examination with balance control}

Normal balance control was found in $93.5 \%$ of the healthy subjects; these subjects were characterised by a normal torsion swing test, caloric and statolith test. So, in only $6.5 \%$ of the subjects a poor correlation was found. In patients $89.3 \%$ of the patients showed an abnormal balance control. Howewer the outcome of the caloric, statolith and torsion swing test could be either normal or abnormal. In 23 out of the 25 whiplash patients balance control was diminished, although caloric and torsion test were normal and only the statolith tests was abnormal in 4 out of 20 whiplash patients. Per definition, the caloric test was abnormal in all patients suffering from peripheral vestibular function loss, but balance control was only abnormal in a lower number of subjects, specially in patients with a gradual onset of function loss (figure 4). Figures 6 and 7 also illustrate the poor correlation between the vestibulo-ocular reflex tests and the dynamic posturography. So, at least no causal correlation could be established in this study between outcome of the caloric, torsion swing or statolith test and TPM, irrespective of the sensory condition and the diagnostic category. It is well accepted that the high frequency VOR is not fully compensated after peripheral function loss, indicating that passive or active head shaking tests are superior to the low frequency torsion swing test to quantify the relevant vestibulo-ocular status. A similar aspect seems to hold now for the vestibulo-spinal reflex: posturography near the limit of stability seems to evaluate the vestibulo-spinal function better than stabilometry or the sensory organisation test, as a clear loss of balance control is observed in patient with vestibular deficits. 


\section{Conclusions}

1. Balance control near the limit of stability was significantly decreased in the majority of chronic whiplash patients with vertigo or balance disorders, and in patients suffering from Ménière's disease or known with a central compensated peripheral vestibular function loss.

2. More than $89.3 \%$ of the patients could be classified with diminished balance control.

3. Based upon their balance control no discrimination could be made between the four different patient groups.

4. No clear cut correlation was found between the outcome of the balance control test and the outcome of the torsion swing test, the statolith function test and the caloric test. 


\section{References}

1. Keim RJ. Clinical comparisons of posturography and electronystagmography (1993). Laryngoscope 103:713-716.

2. Black FO, Shupert CL, Horak FB. Abnormal postural control associated with peripheral vestibular disorders (1988). Prog. Brain Res. 76:263-275.

3. Bles W, De Jong JMBV. Uni- and bilateral loss of vestibular function (1986). In: Bles W, Brandt $T$ (eds.), Disorders of posture and gait, Amsterdlam: Elsevier Science Publishers. 127-141.

4. Nashner LM, Peters JF. Dynamic posturography in the diagnosis and management of dizziness and balance disorders (1990). Neurol. Clin. 8:331-349.

5. Voorhees RL. Dynamic posturography findings in central nervous system disorders (1990). Otolaryngol. Head Neck Surg. 103: 96-101.

6. Furman JM. Role of posturography in the management of vestibular patients (1995). Otolaryngol. Head Neck Surg. 112: 8-15.

7. Goebel JA, Paige GD. Dynamic posturography and caloric test results in patients with and without vertigo (1989). Otolaryngol. Head Neck Surg. 100: 553-558.

8. Black FO, Nashner LM. Postural control in four classes of vestibular abnormalities (1985). In: Igarashi M, Black F.O. (eds), Vestibular and Visual control on Posture and Locomotor Equilibrium. New York: Karger Publications. 271-281.

9. Hamid MA, Hughes GB, Kinney SE. Specificity and sensitivity of dynamic posturography. A retrospective analysis (1991). Acta Oto-Laryngol. 481:596-600.

10. Norré $M E$, Forrez $G$, Beckers A. Vestibulospinal findings in two syndromes with spontaneous vertigo attacks (1989). Ann. Otol. Rhinol. Laryngol. 98: 191-195.

11. Di Fabio RP. Sensitivity and specificity of platform posturography for identifying patients with vestibular dysfunction (1995). Phys. Ther. 75:290-305.

12. Barin K, Seitz CM, Welling DB. Effect of head orientation on the diagnostic sensitivity of posturography in patients with compensated unilateral lesions (1992). Otolaryngol. Head Neck Surg. 106:355-362.

13. Stribley RF, Albers JW, Tourtellotte WW, Cockrell JL. A quantitative study of stance in normal subjects (1974). Arch. Phys. Med. Rehabil. 55:74-80.

14. EL-Kahky AM, Kingma H, de Jong I, Dolmans M. Dynamic posturography in healthy subjects: evaluation of $\mathrm{COG}$ measuring techniques and individualisation of test conditions. Chapter 2 in this thesis.

15. EL-Kahky AM, Kingma $\mathrm{H}$, de Jong I, Dolmans M. Balance control near the limit of stability at various sensory conditions in healthy subjects. Chapter 3 in this thesis.

16. Kingma $H$, Gulikers $H$, de Jong $I$, Jongen $R$, Dolmans $M$, Stegeman P. Real time binocular detection of horizontal, vertical and torsional eye movements by an infra-red video eye tracker (1995). Acta Oto-Laryngol. 520:9-15.

17. Spitzer WO, Skovron ML, Salmi LR, Cassisy JD, Duranceau J, Suissa S, Zeiss E. Scientific monograph of the Quebec task force on whiplash-associated disorders: redefening "whiplash" and its management (1995). Spine, 20:1 S-73S.

18. Norré ME, Forrez G. Posture testing (posturography) in the diagnosis of peripheral vestibular pathology (1986). Arch. Oto-Rhino-Laryngol. 243: 186-189. 
19. Black FO, Nashner LM. Postural disturbance in patients with benign paroxysmal positional nystagmus (1984). Ann. Otol. Rhinol. Laryngol. 93:595-599.

20. Norré ME. Sensory interaction platform posturography in patients with Ménière's syndrome (1993). Am. J. Otolaryngol. 14:404-409.

21. Morrison $G$, Hawken $M$, Kennard $C_{\text {* }}$ Kenyon G. Dynamic platform sway measurement in Ménière's disease (1994). J. Vestibular Res. 4:409-419.

22. Roos JWP. Posturography in the tilting room (1991). Thesis. Free University, Amsterdam, The Netherlands. 


\section{Chapter 6}

\section{Body movement strategy near the limit of stability, studied by video-posturography in normal subjects and dizzy patients}




\section{Abstract}

Movement strategies in healthy subjects and dizzy patients (Whiplash and patients with diseases associated with labyrinthine deficits) were investigated in various sensory conditions by measuring head and body movements in detail with use of video techniques applied in posturography near the limit of stability. The body sway velocities as revealed by the COG velocity were kept within precise limits: $5-8 \mathrm{~cm} . / \mathrm{s}$ in all subjects by adjusting the amplitude of a pseudo randomly destabilising platform. Normal subjects seem to be able to control their body sway by relatively slow movements of the limbs, trunk and head, whereas patients needed more vigorous movements. All dizzy patients showed significantly more vigorous body movements to maintain the same body sway velocity than healthy subjects, but we observed no significant differences in movement strategy when comparing healthy subjects with dizzy patients. Absolute head velocity but also the head velocity relative to the ankle, knee and hip velocities are significantly higher in patients than in normal subjects in all sensory conditions. These higher velocity can be interpreted as a decreased head stabilisation in space, possible as a consequence of a worsened postural control per se. No indication was found that under the current dynamic conditions a clear ankle or hip strategy is used: the body movements are clearly more complex and all segments are involved in ballance control. It is often suggested that in stabilometry with normal stance most subjects use a pure ankle strategy in contrast to the strategies used under dynamic conditions. We therefore suggest to modify the nomenclature to some extent. We suggest to discriminate between a static and a dynamic strategy. In case of a relatively static condition, the COG can be kept within the cone of stability by a static slow strategy which is predominantly achieved by rotation around the ankle (the well known ankle strategy) which does not allow fast corrections but is energy conservative. In contrast, on a soft surface, on a small base of support, on a moving surface, under more challenging conditions, subjects tend to control the COG by using the principle of action and reaction: fast movement of body parts in one direction result in a opposite movement of the body as a whole. This dynamic strategy is reflected in an increase of angular velocities of the ankle, knee, hip and head but is not limited to the "hip strategy".

\section{Introduction}

It has been hypothesised that body movement strategies supporting erect stance are organised using one or a combination of two basic movement patterns involving predominantly ankle or hip rotations (figure 1) $(1,2)$. Nashner and Peters (3) indicated that the use of these two patterns depends on the configuration properties of the support surface and the position of the Center of Gravity (COG) relative to the limits of stability. A quietly standing person uses predominantly the ankle movement strategy as long as the COG moves slowly within the limit of stability. Also, the support surface has to be longer than the feet and firm enough to alow significant levels of torque about the ankle joint. The hip movement strategy will be more effective when the COG 
moves rapidly, approaches the limit of stability, of when the support surface is short or soft $(1,4,5,6,7)$.
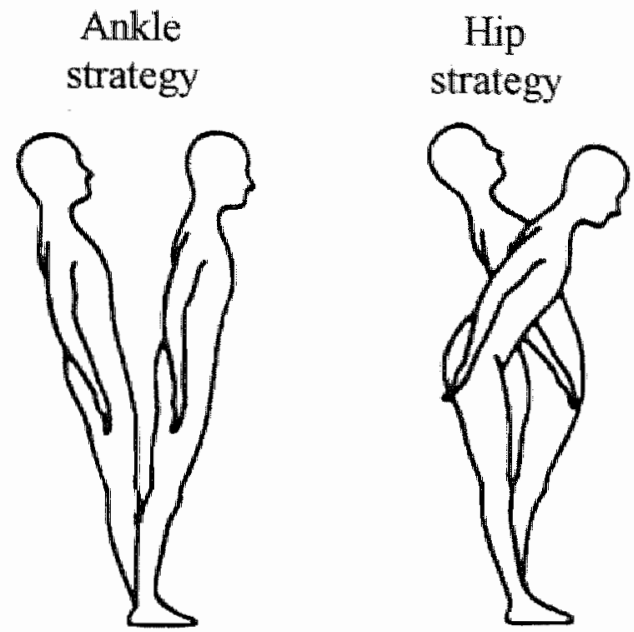

Figure 1.

Schematic representation of the ankle and hip strategies of postural movement (3).

Nashner and co-workers (4) analysed the sensory and mechanical constraints the two strategies and they predicted that a dysfunction of the vestibular system would lead to a destabilisation of the patients and force them to use different movement strategies. This hypothesis could account for the observation that patients with bilateral vestibular loss do not make effective use of hip strategy, even when provided with normal proprioceptive and visual inputs $(8,9)$. So, according to Nashner and Peters (3) patients may rely exclusively on a single strategy -ankle or hip- to maintain balance, even when it is inappropriate for the task condition. This abnormal strategy might reduce postural stability. For example, a patient may fall when he uses the ankle strategy when the COG is located beyond the limit of stability; he should use hip strategy to quickly reposition the body back to equilibrium. Also, a patient will expose an unnecessary large body sway when he depends on the hip strategy only although the COG is located near the centred position. For example, according to Hamid (personal communication, 1995), patients with positional vertigo will try to reduce head movements as much as possible by depending mainly on the hip strategy.

Nashner, (personal communication, 1995), also mentioned that head movements in relation to body movement are important to be observed in dizzy patients. This view is based upon the idea that a vestibular function loss will affect both head stabilisation as 
well as balance control including the movement strategies (4). Additionally, during balance control by either ankle or hip strategy, the head will generally rotate relative to gravity. Decreasing or increasing this head rotation will increase or decrease the angular acceleration inputs to the vertical semicircular canals and the gravity component of the linear acceleration to the otoliths. It was therefore predicted that patients with westibular diseases are forced to use different patterns of head and body movements (4).

Discrimination between hip and ankle strategy is generally made with use of a force platform. Large shear forces with high frequencies are supposed to indicate predominantly hip strategy, whereas small shear forces with low frequencies point to the use of the ankle strategy. Although this measuring technique is elegant and simple, it is less reliable in case of a moving support (see chapter 2 of this thesis) and does not allow to analyse the movements of the feet, lower legs, upper legs, trunk and head separately.

The purpose of this study was to analyse movement strategies in healthy subjects and dizzy patients by measuring head and body movements in more detail with use of video techniques. The major research question in this paper is to verify the hypothesis that analysis of movement strategies allows detection of a vestibular function loss.

\section{Subjects}

77 normal healthy subjects participated in this study ( 36 males and 41 females, 17-68 years old, mean age 26.1 years) and 55 patients (20 males and 35 females, 18-74 years old, mean age 45.7) with persistent complaints of instability and/or vertigo for more than nine months. Only healthy subjects were included that showed a normal otoneurological status and in which standard vestibular testing (spontaneous, gaze, saccades, optokinetic, smooth pursuit, torsion swing, calorics) revealed no abnormalities. Also body sway velocity, body sway area, as well as the associated Romberg coefficients of all subjects as revealed by computerised stabilometry had to be within the normal limits. The subjects had no history of neurologic or musculo-skeletal problems that would affect their balance and orientation. They had no history of episodes of dizziness or underwent any ear operation and were not taking any medication at the time of testing. Onlly subjects were included that considered themselves as physically moderately active (incidental but no regular sport activities).

Only dizzy patients were included that showed a normal neurological status, normal outcome of the spontaneous, gaze, saccadic, optokinetic, and smooth pursuit test. In some patients abnormalities were found with the torsion swing and/or caloric test. The patients had no history of musculo-skeletal problems that would affect their balance and orientation. The patients did not underwent any ear operation and were not taking any medication at the time of testing. The diagnostic profile of the patients and the results of the standard vestibular examination is listed in table 1. The diagnosis definite Ménière's disease was made in agreement with the guidelines of the AAOHNS 1995 classification. Seventeen Ménière's disease patients showed to have a caloric hypo- 
reflexia. All whiplash patients had persistent complaints of instability after rear-end motor vehicle collisions. All of them were considered as chronic cases according to the criteria of the Quebec task force on Whiplash-Associated Disorders $(10,13)$. The results of standard vestibular examination are listed in table 2.

\begin{tabular}{|l|l|}
\hline \multicolumn{1}{|c|}{ Diagnosis \& Group } & \multicolumn{1}{c|}{ Nr. of patients } \\
\hline Dizzy & $55(100 \%)$ \\
Normal calorics & $11(20 \%)$ \\
Caloric hyporeflexia & $44(80 \%)$ \\
Whiplash injury & $11(20 \%)$ \\
\hline
\end{tabular}

Table 1. Diagnostic profile of the patients

\begin{tabular}{|c|c|c|c|}
\hline $\begin{array}{l}\text { Spontaneous / gaze test } \\
\text { Smooth pursuit test } \\
\text { Saccadic test } \\
\text { Optokinetic test }\end{array}$ & \multicolumn{3}{|c|}{$\begin{array}{l}\text { Normal } \\
\text { Normal } \\
\text { Normal } \\
\text { Normal }\end{array}$} \\
\hline $\begin{array}{l}\text { Caloric test } \\
\text { peripheral vest. loss } \\
\text { Ménière's disease } \\
\text { Whiplash injury }\end{array}$ & $\begin{array}{l}\text { Unilateral: } 34 \\
\qquad \begin{array}{c}20 \\
14 \\
0\end{array}\end{array}$ & $\begin{array}{c}\text { Bilaterall: } 10 \\
7 \\
3 \\
0 \\
\end{array}$ & $\begin{array}{c}\text { Normal: } 11 \\
0 \\
0 \\
11 \\
\end{array}$ \\
\hline $\begin{array}{l}\text { Rotatory chair test } \\
\text { peripheral vest. loss } \\
\text { Ménière's disease } \\
\text { Whiplash injury }\end{array}$ & \multicolumn{2}{|c|}{$\begin{array}{l}\text { Abnormal: } 13 \\
10 \\
3 \\
0\end{array}$} & $\begin{array}{l}\text { mal: } 42 \\
17 \\
14 \\
11\end{array}$ \\
\hline
\end{tabular}

Table 2. Results of standard vestibular examination of the patients

The criteria for normality used were as follows:

Spontaneous test

Gaze test

Smooth pursuit

Optokinetic test

Torsion swing test

Caloric test: slow component velocity $(\mathrm{SCV})<5 \mathrm{dg} / \mathrm{sec}$. no nystagmus

position gain $>87 \%$, phase $(-3$ to $+3 \mathrm{dg})$ minimal SCV $>23 \mathrm{dg} / \mathrm{sec}$. at $40 \mathrm{dg} / \mathrm{sec}$. stimulation $0.65>$ gain $>.30$, phase $(-10$ to $10 \mathrm{dg}$ ) at $0.1 \mathrm{H} 2$ cold irrigations $50 \mathrm{dg} / \mathrm{sec},>\mathrm{SCV}>8 \mathrm{dg} / \mathrm{sec}$. $80 \mathrm{dg} / \mathrm{sec} .>\mathrm{SCV}>10 \mathrm{dg} / \mathrm{sec}$. warm irrigations $\mathrm{SCV}>10 \mathrm{dg} / \mathrm{sec}$. unilateral weakness $<20 \%$ nystagmus preponderance $<25 \%$

\section{Methods}

The experimental set-up composed of a high resolution $(<0.1 \mathrm{~cm})$ home built real time infrared (IR) video body movement tracking system, a dynamic force plate (T-Post, Jaeger-Toennies GmbH, Wuerzburg, Germany) provided with a device for cooling the 
foot soles (Instrumental Service Maastricht University (ISMU, The Netherlands)), a dual Achilles" tendon vibrator (ISMU, The Netherlands), a dynamic slide projection systern (ISMU, The Netherlands and Jaeger-Toennies GmbH, Wuerzburg, Germany, patent PT1265390) and a modified Nystagliner system (Jaeger-Toennies GmbH, Wuerzburg, Germany) for data-acquisition (set at $50 \mathrm{~Hz}$ per channel) and stimulus control (platform, slide projection, vibrators). The $50 \mathrm{~Hz}$ IR-video tracker system consisted of a video camera focused on the left side of the subjects body. The camera imaged the position of five active IR markers fixed to the ankle, knee, hip and shoulder joints and near the axis of head nodding. The IR marker positions ( $x, y$ in the anteriorposterior plane) were detected in the video frame, grabbed by a personal computer and subsequently transferred to the data-acquisition PC (Nystagliner). A biomechanical model (chapter 2 of this thesis) was used to calculate the position and velocity of the COG in the anterior-posterior plane based upon the marker positions detected. Body $s w a y$ velocity was calculated by differentiating ( 3 points) the digitised position signal. The data-acquisition computer also controlled the dynamic platform that could rotate up to 9 degrees maximum with a maximum angular velocity of 50 degrees/second and maximum angular acceleration of 1400 degrees $/ \mathrm{sec}^{2}$ (maximum delay $18 \mathrm{msec}$.). The rotation axis of the platform coincided with the axis through the ankle joints. The dynamic slide projector imaged (slightly out of focus for optimal visual impact) a random dot pattern on a transwiew screen (width $3.20 \mathrm{~m}$., height $2.40 \mathrm{~m}.), 0,50 \mathrm{~m}$. in front of the subject. Both slide position and orientation, and the focal length of the projection lens was controlled by four computer (Nystagliner) driven synchro motors (maximum delay $2 \mathrm{msec}$.). As a consequence the image could rotate, translate and zoom in, zoom out to 'counteract' any body or head motion relative to the projection screen, or to mimic any motion of the visual reference frame. Besides the event and time control of the test procedures, the software was able to use 8 signals as an input to produce 8 output signals to control platform rotation or slide position according to a user defined transfer function. The vibration was applied through home built vibrators attached to the right and left Achilles" tendon by an elastic strip. The computer controlled vibrator's DC-motor (Simprop Electronic) housed in a cylinder allowing a certain degree of movement to govern the amplitude of the vibration with a maximum frequency of $250 \mathrm{~Hz}(+/-10 \mathrm{~Hz})$ and a maximum amplitude of $0.2 \mathrm{~cm}$. The cold supporting surface consisted of a metal box filled with ice-water (about 1-3 ${ }^{\circ} \mathrm{C}$ ), specially designed to be placed on the dynamic platform without affecting its movement (ISMU, The Netherlands). It must be marked that the position of the rotation exis was affected by this procedure, but was not taken into considerations with respect to the calculations of the performance.

Based upon the video-imaging technique, movement patterns of body parts could be detected. Head stabilisation was quantified by calculating the absolute value of the angular velocity of the line segment connecting the IR markers mounted on the head and shoulder relative to the trunk ( $\sigma$ ) (line segment connecting the markers mounted on the shoulder and hip). Specific hip movements were quantified by calculation of the absolute value of the angular velocity of the line segment connecting the markers placed on the shoulder and hip relative to the absolute angular velocity of the line segment connecting the markers mounted on the hip and knee $(\alpha)$. Specific knee 
movements were quantified by calculation of the absolute value of the angular velocity of the line segment connecting the markers mounted on the hip and the knee to the absolute angular velocity of the line segment connecting the markers mounted on the knee and ankle $(\varepsilon)$. Specific ankle movements were quantified by calculation of the absolute angular velocity of the line segment connecting the markers mounted to the knee and ankle to the absolute horizontal $(\chi)$ (figure 2).

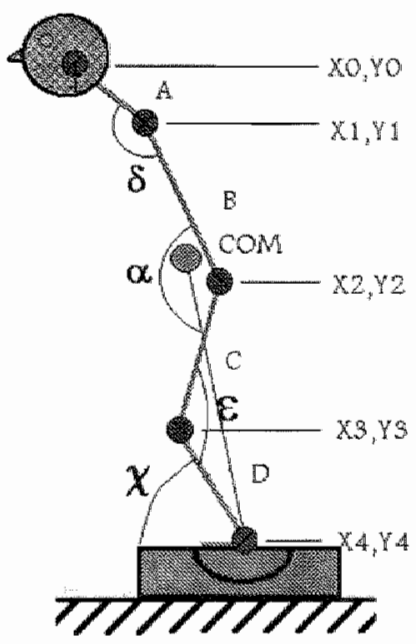

Figure 2.

Schematic drawing of the biomechanical model (see chapter 2 of this thesis) described by Winter (11) and the measured angular velocities.

\section{Experiments}

In all experiments subjects were asked to stand as stable as possible on the dynamic platform, bare feet, arms folded at the waist, looking straight ahead, facing the slide projection screen without leaning forward or backward during each test period of 20 seconds. Temporal vision was blocked by mounting special goggles to the subject. Additionally, all subjects wore a head phone supplied with soft, not distracting music to prevent spatial orientation on auditory cues. $C O G$ position and velocity was continuously measured by means of the video system. The basic platform movement consisted out of 3 mutual harmonically unrelated frequencies $(0.16,0.42,1.15 \mathrm{~Hz})$ with an initial sum of all three amplitudes of 2 degrees. During the duration of the experiment the Amplitude of the platform movement (APM) increased automatically in time with 0.004 degree per $20 \mathrm{~ms}(0.2 \mathrm{dg} / \mathrm{sec}$ ) as long as COG velocity was below $5 \mathrm{~cm} / \mathrm{sec}$. APM was fixed when the subject could maintain the COG velocity within the range from $5-8$ 
$\mathrm{cm} / \mathrm{sec}$. APM automatically decreased with $0.004 \mathrm{dg}$ per $20 \mathrm{msec} .(0.2 \mathrm{dg} / \mathrm{sec}$.) when COG velocity exceeded $8 \mathrm{~cm} / \mathrm{sec}$. APM also decreased whenever $50 \%(3.125 \mathrm{dg})$ of the maximum angle $(6.25 \mathrm{dg}$ ) of forward or backward leaning ("the cone of stability") was reached irrespective the actual body sway velocity, to prevent the subject from falling. Figure was expressed in terms of the tolerated platform movement 'TPM' quantified as the average APM tolerated during the 20 seconds test duration. With an initial APM of 2 degrees and a maximum increase of $0.2 \mathrm{dg} / \mathrm{sec}$. this resulted in a maximum APM of $6 \mathrm{dg}$ after $20 \mathrm{sec}$. and a maximum TPM of $4 \mathrm{dg}$. To study body movement strategy, the angular velocities of the movements; of the head in relation to the trunk, the hip joint (movements of the thigh in relation the trunk), the knee (movements of the thigh to the leg) and the ankle (movements of the leg in relation to the absolute horizontal) were detected and the average velocities over the test 20 seconds duration were calculated. The effect of manipulation the sensory inputs upon the velocities of the head, hip, knee and ankle joints were studied. In all subjects eight different sensory conditions were evaluated as has been previously described (12, chapter 2 and 3 in this thesis).

1) Eyes open, pseudo random platform movement (prm)

2) Eyes open, pseudo random platform movement and sway referenced platform (srp)

3) Eyes open, pseudo random platform movement and sway referenced slide projection (srs)

4) Eyes closed, pseudo random platform movement (ec)

5) Eyes open, pseudo random platform movement and sway referenced platform and slide projection (sip\&s)

6) Eyes closed, sway referenced platform (ec\&srp)

7) Vibration of the calf muscles (vib)

8) Eyes open, pseudo random platform movement and cold platform (col)

\section{Data-analysis}

The APM during each experiment was recorded as a function of time, and TPM and average body part velocities were calculated. Statistical analysis was performed using commercially available software (PC-SPSS version 7.52 for Windows).

\section{Results}

In figure 3 the angular velocities of lower leg to horizontal (ankle), lower leg to thigh (knee), thigh to trunk (hip) and head to trunk (head) in a normal subject are plotted as a function of time. In figure 4 the averaged angular velocities are depicted of ankle, knee, hip and head for normal subjects and patients, for all eight sensory conditions. The relative contributions of the movements of the various body parts, as reflected in the four angular velocities detected, were reproducible ( $100 \% \times \|$ (result 1 -result 2$)$ / (result $1+$ result 2 ) $\mid<16.4 \%$ ) but varied widely between subjects in all four populations of this study. 
Dependence of angular velocities upon the sensory condition in normal subjects and patients

The angular velocities of ankle, knee, hip and head in condition prm were lower than in all other sensory conditions (Wilcoxon Signed Rank Test, $p<0.01$ ). All angular velocities increased minimal in condition srs and maximal in condition ec\&srp (see figures $4 \mathrm{a}-\mathrm{f})$. The angular velocities increased in cranial direction or so to say bottomup $(p<0.05)$. Within most subjects, the average head-trunk angular velocities were not different from the head-vertical (gravitation vector) angular velocities $(p<0.05)$ because of the slow movements of the upper trunk relative to those of the head. The average angular velocity ratio's "head/knee" and "head/hip" in normal subjects did not depend on the sensory condition ( $p>0.05$, see table 3$)$; in contrast the average angular velocity ratio's "head/ankle" were higher in four conditions (ec, ec\&srp, vib, col) than in the reference condition prm $(p<0.05)$. In whiplah patient and even more in dizzy patients, average angular velocity ratio's depended on the sensory condition as well, but in a more complex way $(p<0.05)$.

Angular velocities strategy in patients compared to normal subjects

The absolute angular velocities and the angular velocity ratio's were significantly larger in patients (whiplash patients and dizzy patients with or without a vestibular function loss) than in normal subjects irrespective the sensory condition (MannWhitney U-test, $\mathrm{p}<0.05$ ). The average angular velocity and velocity ratio's did not differ between the three patient groups $(p>0.05$ ) apart from the ratio head $/ \mathrm{knee}$ between whiplash and dizzy patients in condition srp\&s and ec\&srp $(p=0.05)$. See table 3. 


\begin{tabular}{|c|c|c|c|}
\hline & $\begin{array}{l}\text { Ratio-Velocity } \\
\text { Head/Ankle }(\mu \text {-SD) }\end{array}$ & $\begin{array}{l}\text { Ratio-Velocity } \\
\text { Head/Knee }(\mu \text {-SD) } \\
\end{array}$ & $\begin{array}{l}\text { Ratio-Velocity } \\
\text { Head/Hip }(\mu \text {-SD) }\end{array}$ \\
\hline \multicolumn{4}{|l|}{ Normal } \\
\hline $\mathrm{prm}$ & $1.5-0.6$ & $1.4-0.4$ & $1.3-0.4$ \\
\hline $\operatorname{sip}$ & $1.5-0.6$ & $1.4-0.5$ & $1.3-0.4$ \\
\hline srs & $1.5-0.4$ & $1.4-0.4$ & $1.3-0.5$ \\
\hline srp\&s & $1.6-0.6$ & $1.4-0.6$ & $1.3-0.4$ \\
\hline $\mathrm{ec}$ & $1.8-0.5^{*}$ & $1.5-0.4$ & $1.4-0.4$ \\
\hline ec\&srp & $1.7-0.4^{*}$ & $1.5-0.5$ & $1.3-0.4$ \\
\hline vib & $1.8-0.6^{*}$ & $1.6-0.4$ & $1.4-0.5$ \\
\hline $\mathrm{col}$ & $1.7-0.4^{*}$ & $1.6-0.5$ & $1.5-0.5$ \\
\hline \multicolumn{4}{|l|}{ Dizzy } \\
\hline $\mathrm{prm}$ & $2.0-0.4$ & $1.7-0.5$ & $1.4-0.6$ \\
\hline $\operatorname{srp}$ & $2.2-0.6$ & $1.9-0.5 *$ & $1.7-0.4 * 6$ \\
\hline srs & $2.1-0.6$ & $2.0-0.5 *$ & $1.8-0.4 *$ \\
\hline srp\&s & $2.1-0.5 "$ & $1.9-0.4 *$ & $1.8-0.6 *$ \\
\hline $\mathrm{ec}$ & $2.1-0.5 "$ & $1.8-0.4^{\circ}$ & $1.7-0.4 *$ \\
\hline ec\&srp & $2.2-0.6 *$ & $1.8-0.5^{\prime \prime}$ & $1.7-0.4^{*}$ \\
\hline vib & $2.3-0.5 *$ & $1.8-0.5^{\circ}$ & $1.5-0.4$ \\
\hline col & $2.0-0.5$ & $1.6-0.4$ & $1.3-0.6$ \\
\hline \multicolumn{4}{|c|}{ Whiplash } \\
\hline $\mathrm{prm}$ & $2.1-0.5$ & $1.6-0.6$ & $1.6-0.4$ \\
\hline $\operatorname{sip}$ & $2.1-0.5$ & $1.7-0.4$ & $1.5-0.5^{\prime \prime}$ \\
\hline srs & $2.1-0.6^{a}$ & $2.0-0.4 * "$ " & $1.9-0.5 *$ \\
\hline srp\&s & $2.3-0.5 * *$ & $2.3-0.4 * \cdots$ & $1.9-0.5 *$ \\
\hline $\mathrm{ec}$ & $2.1-0.4$ & $1.9-0.5^{*}$ & $1.7-0.4^{16}$ \\
\hline ec\&srp & $2.1-0.6$ & $2.1-0.5 * " 6$ & $1.6-0.6$ \\
\hline vib & $2.3-0.5 *$ & $1.9-0.4 "$ & $1.5-0.4$ \\
\hline $\mathrm{col}$ & $2.1-0.6$ & $1.5-0.5$ & $1.5-0.4$ \\
\hline
\end{tabular}

Table 3. Average ( $\mu$ ) and standard deviation (SD) of the ratio of the angular velocities (head/ankle, head/knee, head/hip) over the eight sensory condition in normal subjects, dizzy patients and whiplash patients. ${ }^{*}$ indicate significant difference compared to the ratio in condition prm ( $p<0.05$, Wilcoxon Signed Ranks test); "indicate significant difference compared to the same velocity ratio in healthy subjects; ' indicate significant difference of the same velocity ratio in whiplash patients compared to dizzy patients. 

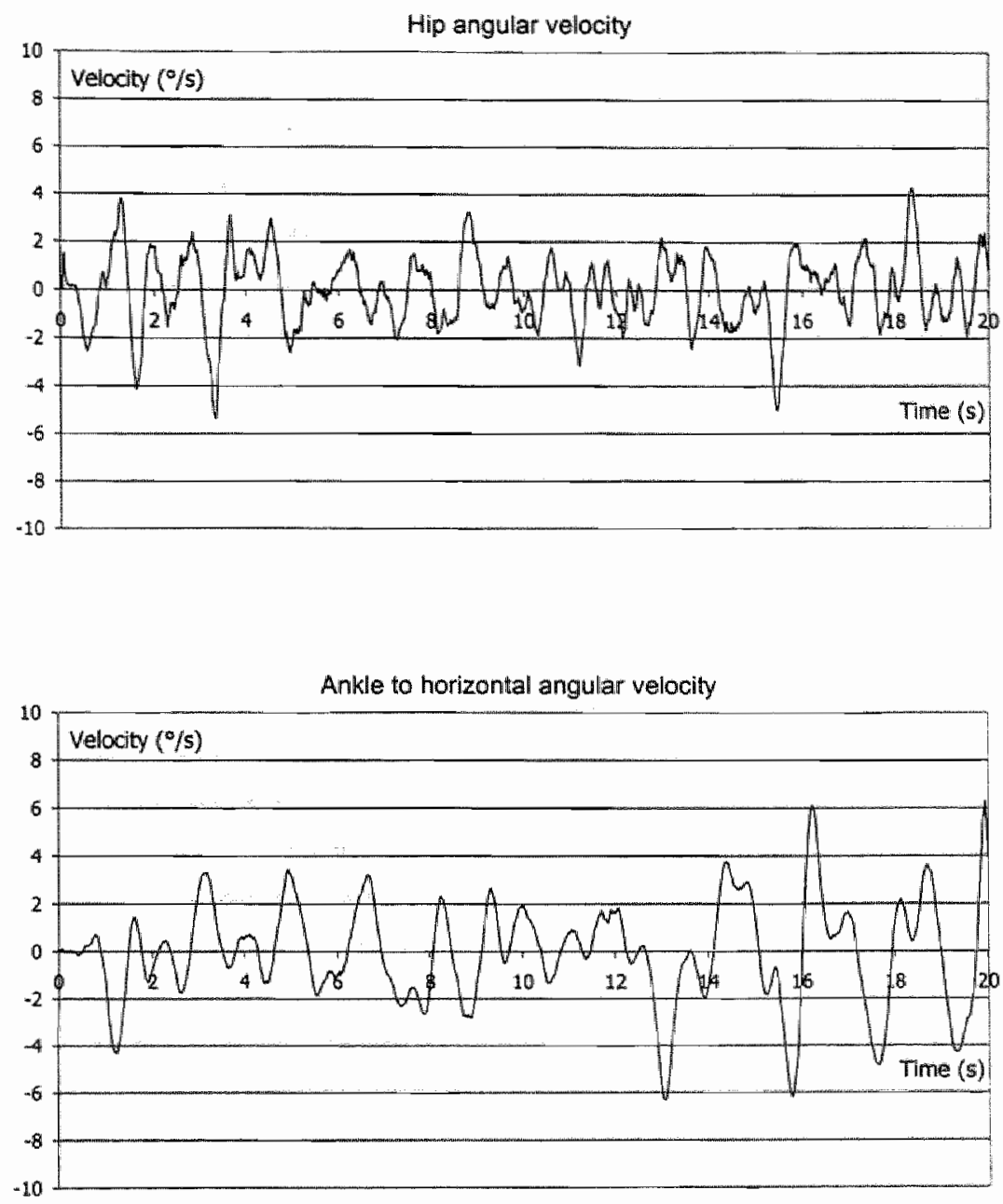

Figure 3a. A typical example of the angular velocities of hip and ankle in a normal subject as a function of time. 


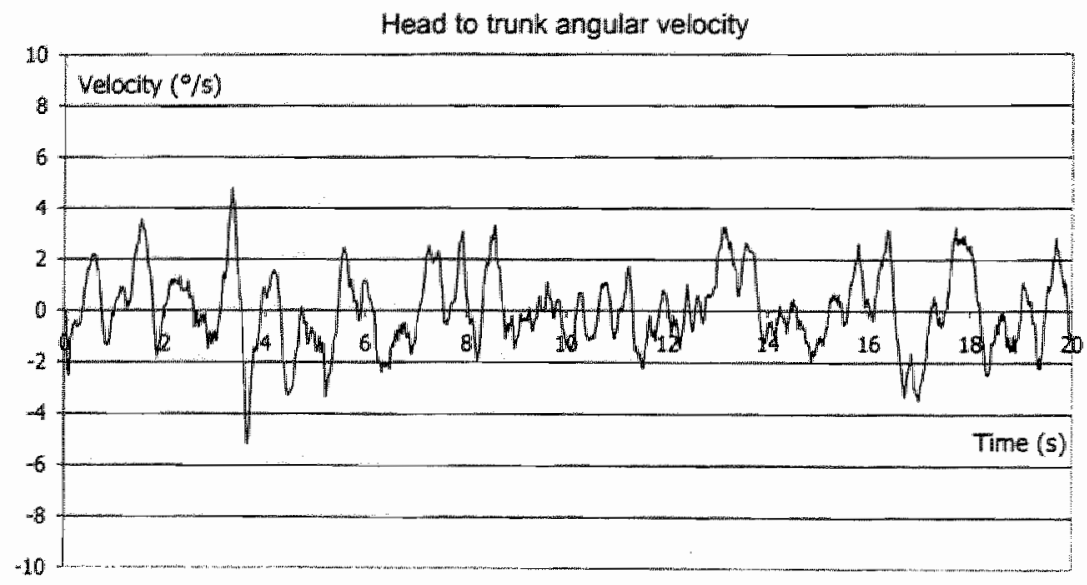

Thigh to lower leg angular velocity

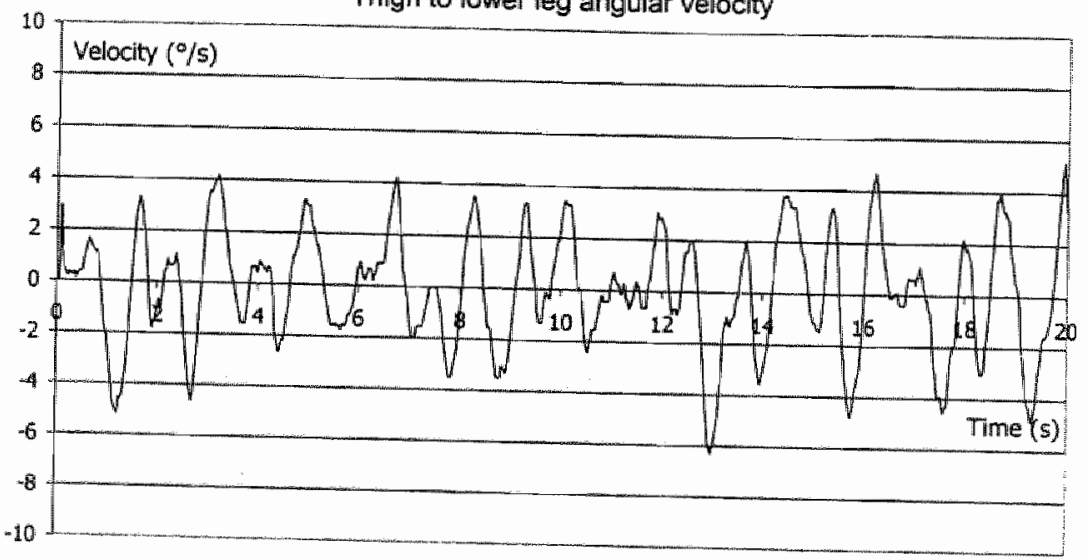

Figure 3b. A typical example of the angular velocities of knee and head in a normal subject as a function of time. 

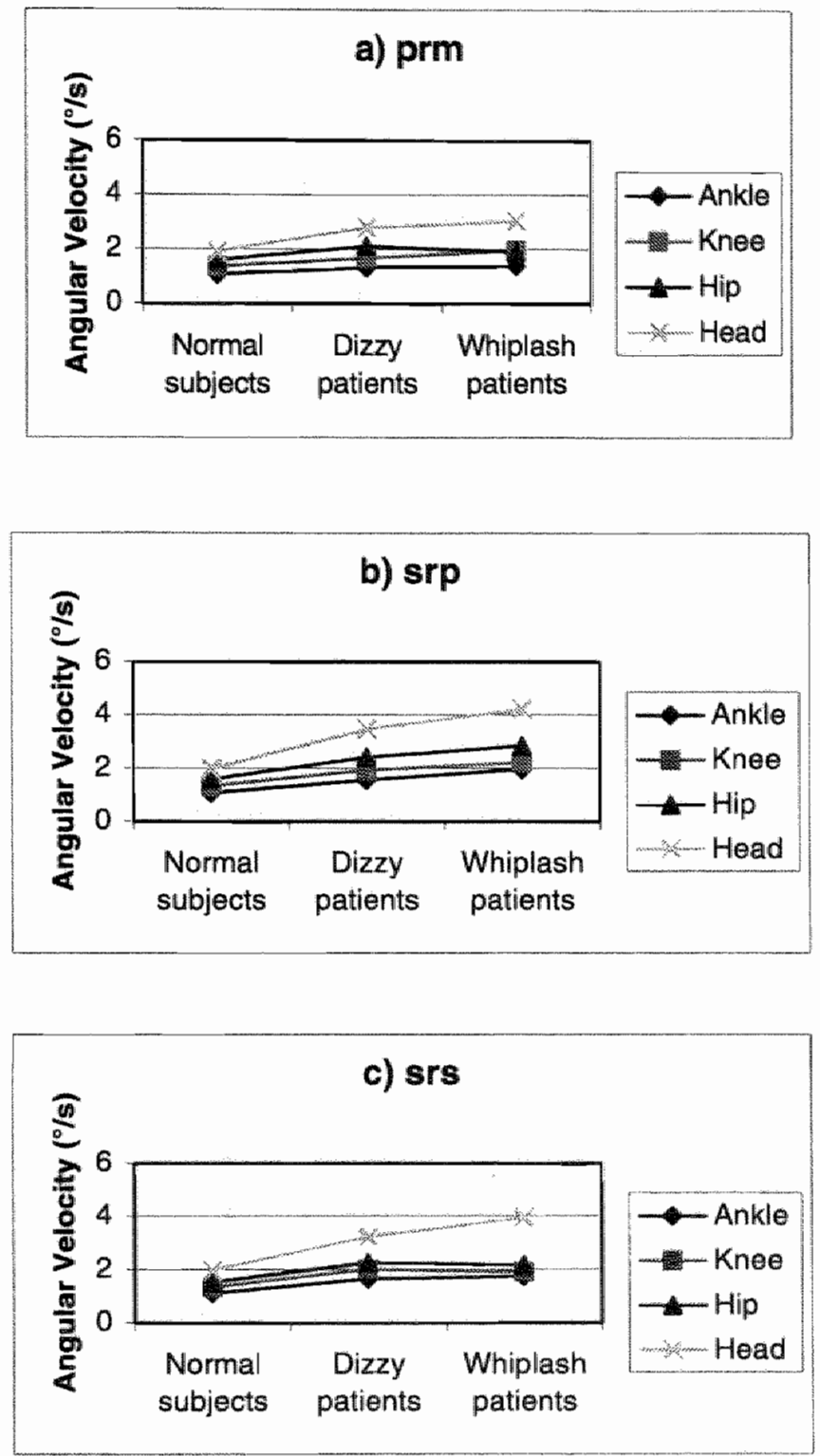

Figure 4a-c. Show the averaged angular welocities of ankle, knee, hip and head for the normal subjects and patients, for 3 sensory conditions.

For abbreviations see text. 

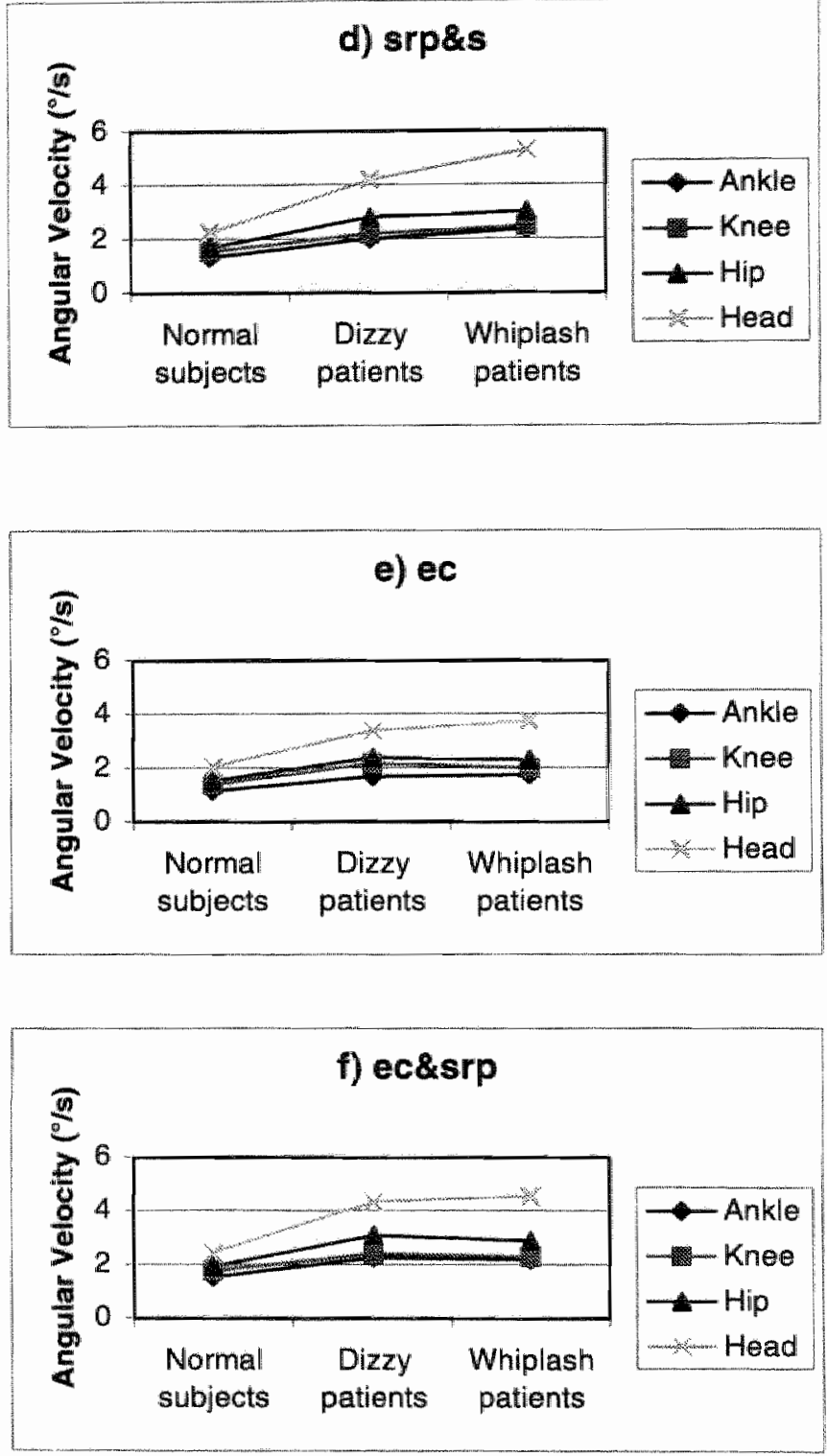

Figure 4d-f. Show the averaged angular velocities of ankle, knee, hip and head for the normal subjects and patients, for 3 sensory conditions. For abbreviations see text. 

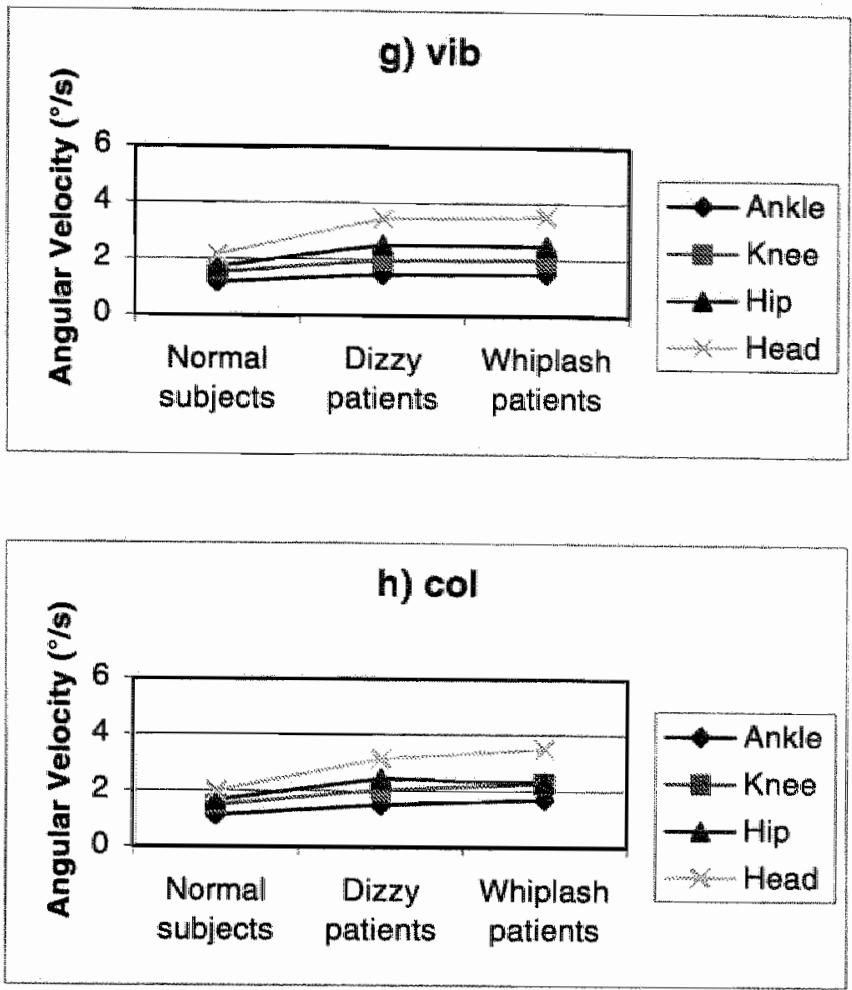

Figure $4 g-h$. Show the averaged angular velocities of ankle, knee, hip and head for the normal subjects and patients, for $2 l$ sensory conditions. For abbreviations see text.

\section{Discussion}

We studied body movement strategy under various sensory conditions in patients and in normal subjects. The body sway velocities as revealed by the COG velocity were kept within precise limits: $5-8 \mathrm{~cm} . \mathrm{s}$ in all subjects by adjusting the amplitude of the moving platform. The large variation of relative contributions of the movements of the various body parts in all four populations of this study hinders a clear discrimination between patients and normals towards movement strategies.

In contrast, we observed a striking difference in the group averages of the angular velocities of ankle, knee, hip and head between normal subjects and patients, and between the various sensory conditions. As the body sway velocity of all subjects was 
kept within the same limits, we conclude that normal subjects seemed to be able to control their body sway by relatively slow movements of the limbs, trunk and head, whereas patients needed more vigorous movements. The relative contribution of the ankle, knee and hip movements to balance control is very similar in normal subjects compared to patients, and does not depend upon the sensory condition. So despite the difference in absolute velocities, -on average- a rather similar movement strategy is used by patients and normal subjects, within the wide variation of strategies used.

In contrast, a striking difference is observed between patients and normal subjects with respect to the head to trunk angular velocities: the head is worse stabilised in patients than in normals. This worse performance regards the absolute head velocity but also the head velocity relative to the ankle, knee and hip vellocities (table 3 ). The higher absollute head velocity in patients in all sensory conditions (relative to the trunk but also to the gravitation vector) points to a decreased head stabilisation in space, possible as a consequence of a worsened postural control per se. Alternatively, one might also hypothesize that the increased head movements are part of a different active strategy to control posture, which also includes -although to a lesser extent- more active movements of all other body parts related to the ankle, knee and hip joint.

Shupert and co-workers $(8,9,10)$ mentioned that patients with profound bilateral vestibular losses do not make use of the hip movement strategy, even when provided with normal proprioceptive and visual inputs, and that even patients with normal findings on caloric and high frequency rotatory chair tests can show abnormal movement strategies. These observations do not agree with our results, as we do not see that much differences in strategies between patients and normals at all, irrespective the outcome of the caloric test or the torsion swing test. Also, even the patients with the most severe vestibular disorders showed a "hip"strategy (see below). We hypothesize that this discrepancy might be primary related to the differences in the test conditions: less challenging with more impact of motivation and instruction (SOT) versus challenging with less impact of motivation and instruction (this study). The latter condition might force any subject to a more or less an optimal strategy which appeared to be not very different between normals and patients.

Under accurate sensory (functionally appropriate) conditions, all examined subjects made use of a relatively static strategy with low body component velocities, i.e. they executed their anterio-posterior sway through the ankle joints. Under different conflicting sensory conditions with a dynamic destabilised platform, all subjects are forced to make use of movements of all body parts to maintain upright posture.

No indication is found that under the current dynamic conditions a clear ankle or hip strategy is used if these strategies would imply the exclusive or dominant movement of the ankle or hip respectively: the body movements are clearly more complex and all segments are involved in balance control. However we are aware of the fact that the term "hip-strategy" already refers to a combination of movements of various body parts. We therefore suggest to modify the nomenclature to some extent. We suggest to discriminate between a static and a dynamic strategy. In case of a relatively static condition the COG can be kept within the cone of stability by a static slow strategy 
which is predominantly achieved by rotation around the ankle (the well known ankle strategy) which does not allow fast corrections but is energy conservative. In contrast, on a soft surface, on a small base of support, on a moving surface, under more challenging conditions, subjects tend to control the $C O G$ by using the principle of action and reaction: fast movement of body parts in one direction result in a opposite movement of the body as a whole. This dynamic strategy is reflected in an increase of angullar velocities of the ankle, knee, hip, head and upper limbs but is not limited to the "hip strategy".

The so-called neuromuscular theory is one of the frequently used explanations in patients with whiplash injury to account for the symptoms $(13,14)$. The most common complaint of whiplash patients is pain in the neck and might point to a dysfunction of the neck, be it with a muscular, proprioceptive, or neuro-muscular origin. As a consequence, one might expect a worse head stabilisation in whiplash patients. The results of this study indeed indicate a poor head stabilisation in whiplash patients, although not worse than in the other two patient group. However, the large interindividual variabillity does not allow a discrimination between a whiplash patient from the normal population, which limits the direct clinical relevance of this finding. Nevertheless, a poor head stabilisation is a severe handicap during many daily life activities and might play a role in the cognitive disorders that are frequently reported in whiplash patients.

\section{Conclusion}

We found no indications for a clear hip or ankle strategy under dynamic conditions where subjects are forced to maintain balance near the limit of stability. In general, during dynamic movements, the cephalic body parts move with higher velocities than the caudal body parts. We observed that any subject, patient or non-patient may, use a wide range of movement strategies involving movements of all body parts. Also, no indication was found that patients with vestibular dysfunction or whiplash patients made use of other movement strategies than normal subjects. On the other hand, it was clearly observed that patients needed higher velocity body movements to control balance than normal subjects, and that -on average- head angular velocities were higher in patients compared to normal subjects. The latter aspect is interpreted as pointing to a worse head stabilisation in patients. 


\section{References}

1. Horak FB, Nashner LM. Central programming of postural movements: adaptation to altered support surface configurations (1986). J. Neurophysiol. 55:1369-1381.

2. Nashner LM, McCollum G. The organization of human postural movements: a formal basis and experimental synthesis (1985). Behav. Brain Sci. 8:135-172.

3. Nashner LM, Peters JF. Dynamic posturography in the diagnosis and management of dizziness and balance disorders (1990). Neurol. Clin. 8:331-349.

4. Nashner LM, Shupert CL, Horak FB, Black FO, Organization of posture control: an analysis of sensory and mechanical constraints (1989). Prog. Brain Res. $80: 411-418$.

5. Moore SP, Horak FB, Nashner LM. The effect of prior leaning on human postural responses (1991). In: NeuroCom International Inc. Equi Test Data Interpretation Marnual. Clackamas, Ore..

6. Horak FB, Nashner LM, Diener HC. Postural strategies associated with somatosensory and vestibular loss (1990). Exp. Brain Res. 82: 167-177.

7. Diener HC, Dichgans L: Long loop reflexes and posture (1986). In: Bles W, Brandt $T$ (eds.), Disorders of posture and gait, Amsterdam: Elsevier Science Publishers. 41-51.

8. Shupert CL, Nashner LM, Horak FB, Black FO. Coordination of the head and body in standing posture in normals and patients with billaterally reduced vestibular function (1987). Soc. Neuros. Abstr. 13:352.

9. Shupert CL, Black FO, Horak FB, Nashner LM. Coordination of the head and body in response to support surface translations in normals and patients with bilaterally reduced vestibular function (1988). In: Amblard B, Berthoz A, and Clarac F. (Eds.), Posture and Gait: Development, Adaptation and Modulation, Amsterdam: Elsevier,. 281-289.

10. Shupert CL, Horak FB, Black FO. Abnormal postural coordination in patients with distorted vestibular function (1988). Soc. Neuros. Abstr. 14:65.

11. Winter DA. Biomechanics of human movement (1979). New York: Wiley \& Sons. 43-64. ISBN: 0-471-03476-2.

12. EL-Kahky AM, de Jong I, Dolmans M., Kingma H. Dynamic posturography in healthy subjects: evaluation of COG measuring techniques and individualisation of test conditions (1998). Chapter 2 in this thesis.

13. Macnab I. The 'whiplash syndrome' (1971). Orthop. Clin. North Am. 2:389-403.

14. Spitzer WO, Skovron ML, Salmi LR, Cassisy JD, Duranceau J, Suissa S, Zeiss E. Scientific monograph of the Quebec task force on whiplash-associated disorders: redefening "whiplash" and its management (1995). Spine, 20:1S-73S. 
Chapter 7

\section{General Discussion}


Advanced computerized posturography is nowadays widely used to quantify several aspects of balance control $(1,2)$. However, the methods applied in the commercially available systems only yield functional information about the vestibular, proprioceptive and visual contributions towards balance control. Besides, the information gained in this way is characterized by considerable inter-and intra individual variability and to our opinion contributes less to the clinical diagnosis than the patient's history and a thorough evaluation of the VOR despite the large variety of opinions in literature (314).

The aum of this study was to develop and evaluate a technique for analysis of movement strategies and for quantification of the contributions of three sensory systems towards balance control.

\section{Design of the equipment and method}

We first developed the experimental set-up for an adequate detection of body position and velocity. Many techniques are available for detecting the COG of which the force platform seems to be the one that is the most frequently used. Yet, force platforms primary record the center of foot pressure as well as the reaction forces (15). The latter aspect hinders a precise detection of the position of the COG under dynamic conditions: already a force exerted to the platform by the toes of a fully stable subject will be recorded by a force platform as a possible movement of the COG. Moreover, this detection technique does not probe the movements of the various body segments separatelly. Often sensors mounted in the platform to detect shear forces are therefore used to discriminate between ankle and hip movements. Through this study we found that an angular rate sensor is more accurate in recording the shift of the COG and consequently the body sway velocity as well (chapter 2). However, real accurate estimation of body movements requires the use of multiple angular rate sensors or multiple IR markers with video-techniques in combination with a bio-mechanical model for a correct interpretation of the sensed signals.

The video-technique with multi marker tracking was used throughout this work as it showed to be a simple and reliable method to detect the body sway velocity and position under dynamic conditions. This technique is widely used in gait analysis. The major inaccuracies of the method are associated with problems in positioning the markers on the anatomical reference points and are related to limitations of the biomechanical models used to interpret the marker movements in terms of active the forces and momenta. The same limitations occur when several multi-axial accelerometers are fixed to the body segments or in case of position detection by magnetic induction. The latter technique is becoming more and more popular. These systems make use of $6 \mathrm{D}$ detection: the position ( $3 \mathrm{D}: \mathrm{x}, \mathrm{y}, \mathrm{z}$ ) and orientation (3D: $\alpha, \beta, \gamma)$ of a body segment in space. The sensors, accelerometers or small 3D magnetic coils, are fixed to the body segments to detect position and orientation of the segment. In table 1 some characteristics of the various $6 \mathrm{D}$ systems are summarized. 


\begin{tabular}{|c|c|c|c|c|c|}
\hline & $\begin{array}{l}\text { Position } \\
\text { detection }\end{array}$ & $\begin{array}{l}\text { Velocity and } \\
\text { Acceleration } \\
\text { detection }\end{array}$ & $\begin{array}{l}\text { Spatial } \\
\text { resolution }\end{array}$ & $\begin{array}{l}\text { Temporal } \\
\text { resolution }\end{array}$ & General remarks \\
\hline $\begin{array}{l}\text { Video }+ \\
\text { markers }\end{array}$ & Yes & $\begin{array}{l}\text { Yes: achieved by } \\
\text { differentiation; } \\
\text { decreased signal } \\
\text { lo noise ratio }\end{array}$ & $\begin{array}{l}<1 \mathrm{dg}^{*} \\
<0.5 \mathrm{~cm}{ }^{*}\end{array}$ & $\begin{array}{l}\text { Typical: }<10 \mathrm{msec} \\
\text { Advanced: }<2 \mathrm{msec}\end{array}$ & $\begin{array}{l}\text { Spatial resolution } \\
\text { depends on range of } \\
\text { motion; "hidden' markers } \\
\text { might complicate } \\
\text { interpretetion; detection } \\
\text { of forces and momenta } \\
\text { depend on biomechanical } \\
\text { model; convenient small } \\
\text { markers }\end{array}$ \\
\hline $\begin{array}{l}\text { Accelerom } \\
\text { eters }\end{array}$ & $\begin{array}{l}\text { Poor: drift } \\
\text { induced by } \\
\text { integration }\end{array}$ & $\begin{array}{l}\text { Accel: optimal } \\
\text { Velocity: drift } \\
\text { Induced by } \\
\text { Integration }\end{array}$ & $\begin{array}{l}<1 \mathrm{dg} / \mathrm{s} 2 \\
<0.5 \mathrm{~cm} / \mathrm{s} 2\end{array}$ & $<1 \mathrm{msec}$ & $\begin{array}{l}\text { Limited range of motion } \\
\text { because connection } \\
\text { subject-machine is } \\
\text { generally required; wirng } \\
\text { and "heawy" body fixed } \\
\text { equipment; casy access to } \\
\text { force detection. }\end{array}$ \\
\hline $\begin{array}{l}\text { Magnetic } \\
\text { coils }\end{array}$ & Yes & $\begin{array}{l}\text { Yes: achiewed by } \\
\text { differentiation; } \\
\text { decreased signal } \\
\text { to noise ratio }\end{array}$ & $\begin{array}{l}<1 \mathrm{dg} * * \\
<0.2 \\
\mathrm{~cm} . * *\end{array}$ & $\begin{array}{l}\text { Typical: }<20 \text { msec } \\
\text { Advanced: }<5 \\
\text { msec }\end{array}$ & $\begin{array}{l}\text { Limited range of motion } \\
\text { because connection } \\
\text { subject-machine is } \\
\text { generally required and } \\
\text { because of limited emitter } \\
\text { range: } \\
\text { Spatial resolution depends } \\
\text { on range of emitter range } \\
\text { **; detection of forces } \\
\text { and momenta depend on } \\
\text { bio-mechanical model; } \\
\text { Large and heavy sensors }\end{array}$ \\
\hline
\end{tabular}

Table 1. Summary of some characteristics of the various $6 D$ systems.

All systems are able to detect lateral as well as forward-backward and up-down movements. This study focussed onto movements in the sagittal plane as is done in most studies. In contrast, the elegant research performed by Bles with respect to balance control is focussing more onto lateral sway (16). In these study a tilting room is used and appears to be a specific otolith-visual interaction test. The work of Bles clearly indicate that balance control should be studied in three dimensions (16). Most likely most research has been performed two-dimensional to allow sufficient simplification for theoretical modeling and to reduce the variability. However, it becomes more and more evident that balance control strongly depends on the precise context in which it is studied, and that extrapolation of results from one condition to another seldom hold. As a full $6 \mathrm{D}$ detection of movements is now technically possible, we would promote to study in the near future posture in six dimensions accordingly. With help of the $4 \mathrm{D}$ video technique, we were able to explore balance control in the sagittal plane in more detail as compared to the use of a force platform. We observed that body sway velocity alone is not a sufficient indicator for the postural system capabilities, but that absolute body position relative to the absolute vertical is also 
essential in the evaluation of balance control. But even more striking, we observed that the study of movement strategies requires a multi-segment analysis.

\section{Intra-individual variability: aspects of habituation and training}

Based upon a number of pilot studies, we concluded that it was necessary to cope with the large range of possible postural capabilities of subjects, and also with the impact of motivation and instruction upon performance. The basic approach chosen was to investigate the balance system under a physiological sensory condition that would be a real challenge for any individual. This idea was based upon the hypothesis that sensory input and motor control is used maximally only, when we are really forced to balance near the limit of stability. Using the pseudo-randomly moving platform as an automatic personal adjustable "debalancing" device, we observed that reproducibility was optimized indeed, and thanks to the improved reproducibility we were able to confirm that balance control does differ from person to person because the differences (inter-individual variances) found were slightly larger than the test-retest variations.

The concept of vestibular rehabilitation therapy is of value in patients with peripheral vestibular disorders, and its purpose is to provide maximal proprioceptive and visual input to expedite vestibular compensation. From birth, the multiple information required for balance is integrated and stored in a "data center" which is thought to be in the reticular formation of the brain stem. New afferent information is constantly compared with these data and under normal circumstances immediate recognition of different sensory patterns enables vestibular activity to occur at a subconscious level. It would seem reasonable to hypothesize that, by carrying out head and balance exercises, the dizzy patient repeatedly presents the brain with the data necessary for new sensory patterns to be learned, following an alteration in the vestibular input. According to many authors this learning process continues until a new pattern of information is developed in the storage mechanism which permits recognition of the unusual input, consequent upon labyrinthine pathology. Posturography as a tool, static or dynamic, have been used to assess balance control in healthy subjects and in patients before and after vestibular training. Normal subjects and patients with peripheral vestibular disorders reduce their body sway over a ten-day training session by visualization of their COG during computerized feedback of their body sway in the anterior-posterior direction (17). Shumway-Cook and Horak used a force platform connected to a microcomputer to assist hemiplegic patients in visualizing their center of pressure, and used this information to reduce load asymmetries in stance $(10)$. These treatment strategies involving visual feedback exercises require training over repeated sessions; therefore a practice effect is introduced, which must be taken into account when evaluating any rehabilitative out come of therapy (17). Hamman et al used the Balance Master (modified Equi-test system) to train and to assess balance control among healthy individuals before and after balance training (17). They reported successful results, in the dynamic postural conditions, again it might be also a practice effect to the test condition is the cause of the subjects" improvement. As measurement of body sway and body position are used as indicator of postural stability under several conflicting sensory conditions. In this study, body sway velocity and position measurement indicated that balance training had no effect upon the healthy subject's postural 
capabilities. So dynamic posturography is not the suitable tool to measure the training outcome. In another words body sway velocity and body position is not the suitable parameter which would indicate the status of the postural system This result is in agreement with some authors who observed upon training significant improvement in the ability of subjects to follow the movement of his/her own COG displayed on a screen (visual feedback training) but could not find any significant improvement in performance on the static postural tests. We interpreted this such that dynamic posturography predominantly quantifies the postural performance on the diagnostic test or training equipment (balance master) with however a poor relation with daily life postural abilities. We observed in this study that training of healthy subjects on a balance bar improved performance on the bar, but hardly affected performance on the posturography test. So, the new method is also not sensitive enough to detect minor differences between healthy subjects and slight improvements in balance control. This aspect could be explained in more detail as follows. Most healthy subjects did not experience the basic test condition (prm) as really challenging; they felt secure and kept on learning during the 20 seconds test duration. When the degree of challenge was increased (higher and/or narrower velocity windows), most subjects could only cope for a short while with the destabilized platform, dropped in performance for a while, increased in performance and so on, but did not reach a steady state. We interpret this now such that the normal balance control system is able to cope with really challenging situations near the limit of stability only for a relatively short while. However it is able to learn from this short challenging experience extremely fast, adapts and is ready to cope with an even more challenging situation. Therefore, as long as the healthy subject feels secure and self confident he will be able to improve his performance until a limit is reached that surpasses by the challenging abilities of our platform. Only in case of misleading or abolishing the sensory sub systems, quite a number of healthy subjects do reach their maximum performance, reflected in a steady state (plateau) within 20 seconds. Obviously many "dizzy" patients feel less secure and self confident on the dynamic platform compared to healthy subjects. This aspect, irrespective the quality of the integrity of the balance control system, might account for the lower performance in patients. In agreement with this interpretation, we observed that not only vestibular rehabilitation, but also reassurance of patients (e.g. based upon the negative outcome of a complete vestibular examination), led to slight but significant improvements of performance. Evaluation of balance control near the limit of stability proved to be a sensitive test for identification of malingerers. Malingerers appeared to be characterized by body sway velocities that are not positively correlated with the magnitude of the debalancing base of support and show inconsistent not reproducible responses. Nevertheless, we are aware of the implications: the current interpretation of the test results limits the usefulness of the dynamic posturography below but also near the limit of stability for clinical application.

\section{Manipulation of the proprioceptive input}

We quantified the impact of most frequently used methods to disturb the proprioceptive system (a sway referenced platform, vibration of the calf muscles, chilling of the foot soles) with the new approach. Vibration of the calf muscles affected balance control 
more than the other two options. However, the results indicated also that none of these methods were able to abolish proprioceptive input completely. This result was expected as there are many structures distributed all over the body to provide proprioceptive information. Corpuscles of Ruffini situated in the capsules of the articulations are providing information about the direction and velocity of movements; articular position is provided through the articular ending of Golgi situated in the articular ligaments; corpuscles of Vater-Paccini situated in the skin provide information about every movement. As this sense is distributed all over and even in the body, it will be virtually impossible to abolish all proprioceptive input to the balance control system. However there is another aspects that complicates the quantification of the proprioceptive contribution towards balance control.

The proprioceptive system has a dual contribution in the process of balance control. First, propriocepsis contributes to the perception of spatial orientation which is a necessary afferent input to the brain to control posture. Second, propriocepsis acts as the sensory part in the feed back loops of the motor control system it self, which should be considered as the effector of the balance control system. So, any form of dynamic posturography that quantifies performance through body movement and aims to quantify the contribution of the "afferent" part of the propriocepsis versus visual and vestibular input, will suffer from this duality. Impairment of propriocepsis will affect posture through both the effector and affector side and reduce performance. The message of this statement is that it might be better to study the sensory contribution to balance control by creating stimulus conditions which hardly require motor abilities: return from dynamic posturography to stabilometry with emphasis on perception of spatial orientation.

\section{Manipulation of the visual input}

We evaluated also two methods to manipulate the visual input to the balance control system (eyes closed and/or a sway referenced slide projection). Eyes closed was more effective in disturbing the postural system. This was evident in both healthy subjects as well as in several patient groups. The eyes closed condition is by far the most simple test to quantify the visual contribution towards balance control. In daily life, visual cues prevent falling through anticipation to obstacles like irregularities of the ground, stairs, holes etc. However, vision is also important for balance control in case of complex, sometimes conflicting sensory conditions like sailing or flying. In case of sensory conflicts, the ability to selectively suppress visual or vestibular cues and to favor the competitive sensory input is an essential feature.

This is precisely the basic rationale behind the implementation of the sway referenced visual surround in the sensory organization test. This condition might inform us about the ability of a subject to suppress misleading visual cues towards spatial orientation. Impairment of this ability is thought to point to a specific class of dysfunction's named "visually dependent subjects" that can be considered as the inverse of visually impaired subjects.

It is widely known that the precise characteristics of the visual scenery determine whether the individual will be misleaded by visual cues. The slide projection used in our research project can be expected to be a less effective visual reference in 
comparison with the "concrete "visual reference applied in the equitest system used by many researchers. The best visual reference is a tilting room as used by Bles and coworkers (see chapter 1) that surrounds the subject completely (16). Another basic problem with any sensory cue is that its impact often depends on the mental setting and the pre-knowledge of the subject, a factor that introduces extra variability in responses. A well known example is that of the passenger sitting in a train that he expects to leave any moment now. When he looks through the window while the train besides him starts to move, the passenger will interpret this moving visual cue such that he thinks that his train is departing, despite the lack of other sensory cues (vestibular, proprioceptive and auditive). The mental setting (expectation to leave) apparently overrules the actual sensory information; we know that these misinterpretations frequently happen, specially in fast changing sensory conditions with a low degree of attention. One might expect that in case of balance control, the impact of pre-knowledge and mental setting, is highly relevant when near the limit of stability. Under such dynamic circumstances, postural adjustments have to be fast forcing the subject to be extremely alert and allowing less time for a critical evaluation of the reliability of the perceived cues. However, we learned from a critical evaluation of several moving visual cues, that most subjects are able to exclude fake visual cues within a few seconds, subsequently neglect them and concentrate upon the alternative reliable sensory inputs. Therefore we think that the slide projection used in this research is indeed a less effective visual reference in comparison with the "concrete" visual reference applied in the equitest system. Nevertheless, the slide projection was found to induce an increase of body sway in stable platform conditions which indicates that the subjects experience the image realistic enough to contribute to spatial orientation. A sway referenced slide projection induced additional instability in patients suffering from a whiplash injury compared to healthy subjects and vestibular deficits' patients. This is interpreted in terms of frequently reported reduced attention and concentration abilities in Whiplash patients. The reduced attention might account for the reduced ability to estimate the reliability of the perceived sensory cues.

\section{Quantification of sensory contribution towards balance control}

A major conclusion from our study is that with concern to balance control, both movement strategy as well as the use of sensory inputs varies widely from individual to individual and that no typical patterns can be observed in healthy subjects or the patients examined through this work.

On an anatomical and physiological basis, it is well known that the pathways of the VOR and VSR are separate. The compensation process for both reflexes is different as well. The three semicircular canals and the otolithic organs are responsible for both reflexes. Our ability to investigate the system 'precisely' is available mainly through the caloric and the standard rotatory chair tests which however evaluate the function of the lateral semicircular canal only. Other parts of the labyrinth (the vertical semicircular canals and the otolithic organs) are not routinely or precisely investigated. The VSR depends on input from all semicircular canals as well as the otolithic organs. Therefore, implicitly, posturography evaluates the labyrinth as a whole, which might account for the difficulty to have a precise clinical evaluation of balance control. Moreover, the 
westibular system is only one of the many afferent inputs to a complex multi-sensory orientation controlled system and ultimately the vestibular activity produces postural changes only after it has been processed and monitored in the light of other leamed processes. The latter aspect is extremely relevant: a different context or a manipulation of the sensory input will activate other multi-sensoric efference copies and lead to a complete change in weighting factors of the sensory inputs towards the brain (see chapter 5). These aspects hinder us to uncap this complex multi-sensoric system just by dynamic posturography. Actually we therefore consider posturography as a test that merely supplies functional information about balance control in the test context but is not able to quantify the contributions of the sensory input.

\section{Vestibular deficits and movement strategies}

The maculae induce muscle contractions that produce negative geotropic movement or forces compensating for steady changes in the direction of the force of gravity. Additionally, upon excitation, semicircular canals and otolithic organs mediate transitory contractions of the muscles. One might therefore expect that patients with vestibular diseases would have different patterns of head and body movements. Yet, through this study we did not find any difference in the head or the body movements in dizzy subjects compared with healthy subjects. Additionally, we could not confirm reports that the maintenance of postural sway in the anterio-posterior sway is by hip and or ankle movements (strategies). We indicated that human control balance in all possible planes with all possible body segments and joints, and that the concept of hip and ankle strategy can be considered as an artifact of the fact that balance control is then described in the sagittal plane alone. 


\section{References}

1. Black FO. Vestibulospinal function assessment by moving platform posturography (1985). Am. J. Otol. Suppl. 39-46.

2. Nashner LM, Shupert CL, Horak FB, Black FO. Organization of posture controls: an analysis of sensory and mechanical constraints (1989). Prog. Brain Res. 80:411418.

3. Black FO, Nashner LM. Postural control in four classes of vestibular abnormalities (1985). In: Igarashi M, Black F.O. (eds), Vestibular and Visual control on Posture and Locomotor Equilibrium. New York: Karger Publications. 271-281.

4. Mirka A, Black FO. Clinical application of dynamic posturography for evaluating sensory integration and vestibular dysfunction (1990). Neurol. Clin. 8:351-359.

5. Shepard NT. The clinical use of dynamic posturography in the elderly (1989). Ear Nose Throat J. 68:940-957.

6. Norré ME, Forrez G. Vestibulospinal function in otoneurology (1986). ORL J. Otorhinolaryngol. Relat. Spec. 48:37-44.

7. Norré ME, Forrez $G$. Posture testing (posturography) in the diagnosis of peripheral vestibular pathology (1986). Arch. Oto-Rhino-Laryngol. 243:186-189.

8. Norré M.E. Contribution of a posturographic six-testset to the evaluation of patients with peripheral vestibular disorders (1992). J. Vestibular Res. 2:159-166.

9. Black FO, Nashner LM. Vestibulospinal control differs in patients with reduced and distorted vestibular function (1984). Acta Oto-Laryngol. Suppl. 406:110-114.

10. Shumway-Cook A, Horak FB. Assessing the influence of sensory interaction on balance: Suggestions from the field (1986). Phys. Ther. 66:1548-1550.

11. Hamid MA, Hughes GB, Kinney SE. Specificity and sensitivity of dynamic posturography. A retrospective analysis (1991). Acta Oto-Laryngol. Suppl. 481:596-600.

12. Black FO, Shupert CL, Horak FB, Nasher LM. Abnormal balance control associated with peripheral vestibular disorders (1988). Prog. Brain Res. 76:263275.

13. Shupert CL, Nashner LM, Horak FB, Black FO. Coordination of the head and body in standing posture in normals and patients with bilaterally reduced vestibular function (1987). Soc. Neuros. 13:352.

14. Shupert CL., Horak FB, Black FO. Abnormal postural coordination in patients with distorted vestibullar function (1988) Soc. Neuros. 14:65.

15. Berendsen T. Quantitative mechanical analysis of the detection of position of body COG by a force platform (1991). Master Thesis, Technical Uniwersity Twente.

16. Bles W. Sensory interactions and human posture (1979). Thesis, Free University, Amsterdam.

17. Hamman RG, Mekjavic I, Mallinson AI, Longridge NS. Training effects during repeated therapy sessions of balance training using visual feedback (1992). Arch. Phys. Med. Rehabil. 73:738-744. 


\section{Summary}


The aim of this study was to develop and evaluate a technique for analysis of movement strategies and for quantification of the contributions of three sensory systems towards balance control. An introduction to postural control and quantitative methods for evaluation of the postural control system is given in chapter 1 . Upright stance has an enormous significance for the human being, because it frees the hands for productive activity and improves visual orientation. Upright stance and balance in general is regulated by neuromuscular control centres in the brain that depend upon redundant information provided by vestibular, visual and somatosensory input. In the context of balance control, these three sensory systems provide the following information. The vestibullar system senses absolute head acceleration and spatial orientation. The visual system detects motion and orientation of the image of the visual surround projected on the retina. The somatosensory system primarily senses the motions of the lower extremities as well as the force exerted by the feet on the support surface. Therefore, body orientation is detected in three different frames of references. Vestibular input is partly referenced to gravity, visual input is referenced to the visual surround and somatosensory input is referenced to an earth based support surface. Absolute orientation to the earth vertical is therefore derived from the vestibular system only, whereas the other senses provide relative orientation references. A balance system abnormality may occur in the three sensory systems mentioned above, in the motor system involved in executing the appropriate musculoskeletal responses and in the associated neuro-muscular and sensory integration centres in the brain. The complexity of the balance system makes localization of any dysequilibrium problem difficult, even if other causes of instability can been ruled out. Therefore, loss of balance control has intensively been studied through quantification of vestibulo-spinal reflexes. Quantification of vestibulo-spinal reflexes is done by measuring either EMG activity of the postural muscles or more often by measuring body sway. Dynamic posturography was introduced about fifteen years ago where sway referenced platforms were applied to reduce or mislead the somatosensory input in a sophisticated and computer controlled way in combination with a sway referenced visual surround. The ultimate goal was isolation of one or more sensory input systems. Dynamic posturography system has become the standard of posturography world-wide, despite some essential drawbacks that has to be considered as a functional test of balance with limited diagnostic capabilities. In chapter 2 , two of the basic problems with dynamic posturography are addressed and evaluated by studying postural control in 11 healthy subjects. The first problem concerns the fundamental limitation of the force platform as a measuring device applied in posturography. An alternative technique based upon infrared (IR)-video tracking of five markers fixed to the head, shoulder, hip, knee and ankle was developed to calculate the position of the body centre of gravity (COG); this technique is compared with techniques using a force platform or a single body fixed angular rate sensor. The fundamental question is put forward whether force transducers mounted in platforms are adequate sensors to detect COG position specially when applied in dynamic conditions (vigorous moving subjects or platforms). The second problem concerns the poor reproducibility and low specificity of dynamic posturography: test performance is related to the functional status of the subjects balance system, and the subject's motivation and 
interpretation of the test-instruction. "Good" patients appear to perform better than "bad" normal subjects and most subjects show a steady improvement during successive testing (habituation and adaptation). To cope with this problem a technique is introduced to quantify postural control near the limit of stability to force the subject to an optimal performance. We hypothesise that in this way every subject is tested according to the individual abilities and that reproducibility might improve when compared to more static, non individualised or less challenging test conditions.

Measurement technique. The output of the three detection systems and a videorecording of the actual body movements were compared in six different body and/or platform movement conditions. The force transducers detect the centre of pressure of the feet, which induces considerable systematic errors in the calculation of the actual body sway at physiological movement frequencies above $0.2 \mathrm{~Hz}$. We conclude that, the value of dynamic posturography using force platforms should be considered in line with the limited accuracy of this method to detect the COG position under dynamic conditions. Application of one single angular rate sensor neither provides an accurate detection of the COG position nor velocity. In contrast, despite some theoretical limitations related to the biomechanical models to be used, we conclude that the video tracking method produced the most accurate and reliable estimate of the COG movement, specially in case of substantial body movements at frequencies above 0.2 $\mathrm{Hz}$.

Quantification of postural control near the limit of stability. 11 healthy subjects stood with eyes open on a motor driven platform that rotated around the ankle axis pseudorandomly. COG position and velocity were real time monitored by means of the video system. During each test the amplitude of the platform movement was maximised automatically as long as the body movement was in accordance with pre-set COG velocity and COG position limits. Four physically active subjects tolerated higher platform amplitudes than three moderately active subjects; the remaining four relatively inactive subjects tolerated only small platform movements. The test score thus correlated well with the subjects own judgement of balance capabilities in daily life in contrast with the findings of the routine stabilometry that showed normal and not-significantly different postural control in all 11 subjects. Reproducibility of the test scores was within $18 \%$. In chapter 3, the new technique is applied in 97 healthy subjects to explore the perturbation effect upon postural control of sway referencing the supporting platform, of sway referencing the visual surround, of closing the eyes, of vibration of the Achilles tendon and chilling the foot soles. Posture control on average- was maximally affected by closure of the eyes (decrease of performance with about 37\%) and by vibration of the Achilles tendons (about 21\%). The other perturbation techniques applied were less effective. The combination of vibration and a sway referencing platform was the most effective technique used to disturb the proprioceptive input. The effect of chilling the foot soles ( 5 minutes) was not very effective; longer cooling was badly appreciated. Combination of perturbation techniques was often more effective but was not merely a linear summation of the effect induced by the techniques applied separately. Combination of closure of the eyes with a sway referenced platform and wibration was the most effective perturbation technique of postural control $(56 \%)$. The data suggested that vision contributes 
maximum $37 \%$, propriocepsis minimum $26 \%$ and labyrinths maximum $44 \%$ to postural control in healthy subjects, and that depending on the available sensory information and acquired postural control strategies, weighting factors of the sensory inputs may vary within these margins. However, individual data suggested that the contribution of the sensory inputs toward postural control varies considerably between individuals which might limit the clinical relevance of dynamic posturography. In chapter 4 by means of a double blind placebo-controlled study the new posturography technique is applied to evaluate the effect of habituation to the test conditions in 11 healthy subjects (experiment 1) and to explore the effect of specific balance training upon posture control in 56 other healthy subjects (experiment 2). In the first experiment, subjects were tested three times on one day. In the second experiment, 28 subjects were trained on a balancing bar (training group), while 28 subjects performed a pseudo-training (control group). The results show edno significant effect of habituation to the test condition or equipment. Training on the balance bar showed a steady improvement with respect to the balancing abilities on the bar from session to session. The subjects in the 'training' and control group reported no clear change of balance control in daily life activities despite the significant improvement of performance on the balance bar. No effect of balance training was observed upon posture control near the limit of stability as quantified by dynamic posturography. The negative test outcome of dynamic posturography correlated well with the absence of any effect of balance training upon daily life activities but does not correlate with the improvement of performance on the balance bar. We concluded that posturography near the limit of stability is not able to quantify the effect of specific balance training that has no impact on postural control in daily life activities. In contrast, in a previous study, we observed that the test scores obtained by posturography near the limit of stability correlated well with the subjects own judgement of balance capabilities (sports) in daily life. It is suggested, that dynamic posturography primary evaluates motor control and less spatial orientation and is not suited to evaluate specific balance control as a reflection of spatial orientation abilities. In chapter 5 the new technique is applied in four groups of patients suffering from long lasting persistent vertigo (whiplash syndrome $(n=25)$, Ménière"s disease $(n=28)$, acute $(n=28)$ or gradual peripheral function loss $(n=26)$ ) with various abnormalities detected by standard vestibular examination and a statolith function test. Posture control -on average- was maximally affected by closure of the eyes and by vibration of the Achilles tendons. The other perturbation techniques applied were less effective. The combination of vibration and a sway referencing platform was the most effective technique used to disturb the proprioceptive input. No consistent typical pattern was observed that could classify the patients with respect to the history or outcome of the standard vestibular examination: the individual data indicated that the contribution of the sensory inputs toward postural control varies considerably between patients and clearly limits the clinical relevance of this type of dynamic posturography. Postural control near the limit of stability was significantly decreased in the majority of chronic whiplash patients with vertigo or balance disorders, as well as patients suffering from Ménière's disease or known with a central compensated peripheral vestibular function loss. About ninety three percent of the 
patients could be classified with use of the postural control test, specially when the responses on the conditions with a sway referenced vision, and eyes closed (with and without the sway referenced platform) were taken into account. However, based upon their postural control no discrimination could be made between the four different patient groups. In chapter 6 movement strategies in healthy subjects and dizzy patients (Whiplash and patients with diseases associated with labyrinthine deficits) are investigated by measuring head and body movements in detail with use of the video techniques applied in the new posturography technique. All healthy subjects had on average a similar body sway velocity. Normal subjects seem to be able to control their body sway by relatively slow movements of the limbs, trunk and head, whereas patients need more vigorous movements. All dizzy patients showed significantly more vigorous body movements to maintain the same body sway velocity than healthy subjects, but we observed no significant differences in movement strategy when comparing healthy subjects with dizzy patients. Also, head angular velocities were larger in patients than in healthy subjects pointing to a worse head stabilisation or an active role of head movements in balance control. No indication was found that under the current dynamic conditions a clear ankle or hip strategy is used: the body movements are very complex and all segments are involved in balance control. We therefore suggest to modify the nomenclature of movement strategy to some extent. We suggest to discriminate between a static and a dynamic strategy. In case of a relatively static condition, the COG can be kept within the cone of stability by a static slow strategy which is predominantly achieved by rotation around the ankle (the well known ankle strategy) which does not allow fast corrections but is energy conservative. In contrast, on a soft surface, on a small base of support, on a moving surface, under more challenging conditions, subjects tend to control the COG by using the principle of action and reaction: fast movement of body parts in one direction result in a opposite movement of the body as a whole. This dynamic strategy is reflected in an increase of angular velocities of the ankle, knee, hip and head but is not limited to the "hip strategy". In chapter 7, several aspects of postural control near the limit of stability are discussed in terms of clinical relevance and the new method is critically evaluated. It became more and more evident that postural control strongly depends on the precise context in which it is studied, and that extrapolation of results from one condition to another seldom hold. As a full $6 \mathrm{D}$ detection of movements is now technically possible with video-techniques or equivalents, we promote to study in the near future posture in six dimensions accordingly and not only in $4 D$ as has been done mostly. A major conclusion from our study is that with concern to postural control, both movement strategy as well as the use of sensory inputs varies widely from individual to individual and that no typical patterns can be observed in healthy subjects or the patients examined through this work. The current interpretation of all test results point to a limited usefulness of the dynamic posturography below but also near the limit of stability for clinical application. We suggest that it might be better to study the sensory contribution to balance control by creating stimulus conditions which hardly require motor abilities: return from dynamic posturography to stabilometry with emphasis on perception of spatial orientation. 


\section{Samenvatting}


Het in dit proefschrift beschreven onderzoek beoogt een methode te ontwikkelen en te evalueren voor analyse van de bewegingsstrategieën van het houdingsevenwicht en kwantificatie van de bijdragen van de zintuiglijke informatie aan de houdingscontrole.

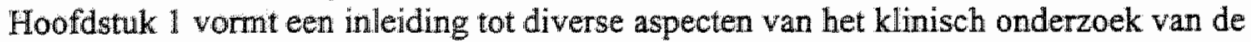
houdingscontrole. Aangegeven wordt dat het rechtop staan en de houdingsbalans geregeld worden door neuro-musculaire centra in de bersenen op grond van redundante informatie vanuit het vestibulair, visueel en somatosensorisch systeem. Het vestibulair systeem zorgt voor informatie inzake de versnellingen van het hoofd en de absolute stand van het hoofd ten opzichte van de zwaartekracht vector. Het visuele systeem detecteert primair de bewegingen en de oriëntatie van het op het netvlies geprojecteerde beeld. Het somatosensorisch systeem detecteert de stand en de bewegingen van alle lichaamsdelen en de kracht die het lichaam uitoefent op de omgeving (meestal de voeten op de ondergrond). In dit kader gaan we niet in op het feit dat ook andere zintuigsystemen via ruimtelijke oriëntatie invloed op het houdingsevenwicht kunnen uitoefenen (bijv. oriëntatie op geluid). De oriëntatie van het lichaam in de ruimte wordt derhalve voornamelijk in drie referentiekaders bepaald. Het vestibulair systeem detecteert de oriëntatie ten opzichte van de zwaartekracht vector, het visuele systeem detecteert de oriëntatie ten opzichte van de visuele omgeving en het somatosensorische systeem detecteert de oriëntatie ten opzichte van het contactoppervlak van het lichaam met de omgeving. Een functiestoornis in een der drie betrokken zintuigsystemen, in het motorisch apparaat, in de motorische of sensomotorische integratie centra in de hersenen, kunnen allen leiden tot stoomissen van de houdingscontrole. De complexiteit van het evenwichtsstelsel is zodanig groot dat een lokalisatie van het functieverlies moeilijk is, zelfs indien andere niet vestibulaire oorzaken van de evenwichtsproblemen kunnen worden uitgesloten.

Ongeveer 15 jaar geleden werd de zogenaamde dynamische posturografie geïntroduceerd waarbij het steunvlak of de visuele omgeving de lichaamsbewegingen kunnen volgen (feed back: "sway referenced platform" en "sway refenced visual surround") zodat de somatosensorische en visuele input wan het houdingscontrolesysteem gemanipuleerd wordt. Met deze zogenaamde Sensorische Organisation Test (SOT) wordt getracht de bijdrage van visuele, somatosensorische en vestibulaire zintuiglijke informatie aan de houdingscontrole te kwantificeren. Deze SOT vormt wereldwijd de standaard voor kwantificatie van de houdingscontrole, ondanks het feit dat vooral het functionele aspect van de houdingscontrole wordt geanalyseerd en de methode maar een beperkte diagnostische waarde heeft.

In hoofdstuk twee worden twee fundamentele problemen bij de dynamische posturografie onderzocht bij een groep van 11 gezonde proefpersonen. Het eerste probleem betreft de beperking van snelheidssensoren, versnellings- of krachtsensoren aangebracht in platforms of op het subject om een nauwkeurige schatting te maken van de daadwerkelijke lichaamsbewegingen. Het met deze technieken verkregen resultaat wordt vergeleken met de resultaten van metingen van lichaamsbewegingen met behulp van een binnen onze vakgroep zelf ontwikkeld infra-rood videocamera systeem dat de lichaamspositie real time meet op grond van de afbeelding van 5 markers aangebracht op de enkel, knie, heup, romp en het hoofd. De resultaten verkregen met de verschillende meettechnieken onder zes verschillende testcondities werden vergeleken. 
Met behulp van krachtopnemers wordt primair het punt van maximale druk onder de voeten bepaald, hetgeen bij lage frequenties nauwkeurig overeenkomt met de bewegingen van het massazwaartepunt van het lichaam, maar bij frequenties boven 0.2 $\mathrm{Hz}$ niet meer. We concluderen dat de dynamische posturografie die gebruikmaakt van een krachtenplatform, beperkingen kent die direct gerelateerd zijn aan onnauwkeurigheden van deze methodiek bij de bepaling van de positie en positieveranderingen van het lichaamszwaartepunt (COG) onder dynamische omstandigheden. Toepassing van een enkele snelheidssensor vlakbij het massazwaartepunt levert geen nauwkeurige schatting van de positie en bewegingen van het COG. Vastgesteld werd dat vooral tijdens snelle platform of lichaamsbewegingen, de video techniek de lichaamsbewegingen het meest betrouwbaar weergeeft, en de enige techniek is die de absolute oriëntatie van de verschillende lichaamsdelen kan kwantificeren. De beperkingen van deze techniek ligt vooral in de onnauwkeurigheden en onzekerheden in het gehanteerde biomechanisch model waarmee de positie van markers vertaald wordt in positie en oriëntatie van lichaamsdelen en het massazwaartepunt.

Het tweede probleem dat in hoofdstuk 2 wordt onderzocht betreft de beperkte reproduceerbaarheid en specificiteit van de SOT. Over het algemeen wordt de score van de subjecten bij de SOT primair bepaald door hun functionele status op het moment van onderzoek; de score wordt echter ook beïnvloed door de motivatie en de instructie van de subjecten. "Goede" patiënten kunnen een beter score hebben dan "slechte" gezonde subjecten. Tevens wordt bij opeenvolgende testen de score vaak steeds beter, wijzend op habituatie of adaptatie en resulterend in een aanzienlijke testretest variabiliteit. Wij hebben in deze studie getracht dit probleem op te lossen door de houdingscontrole te kwantificeren onder condities waar mensen nog net hun evenwicht kunnen bewaren: ieder subject wordt zo gedwongen tot een optimale prestatie, maximaal gebruikmakend maar ook maximaal afhankelijk van alle beschikbare zintuiglijke informatie. We veronderstelden dat deze benadering zou kunnen leiden tot een meting van primair de individuele capaciteiten en dat de reproduceerbaarheid beter zou kunnen zijn dan onder statische minder uitdagende testcondities, waar aandacht, concentratie, instructie en motivatie een belangrijke rol spelen. Bij 11 gezonde proefpersonen (vier fysiek zeer actieve, drie fysiek matig actieve, vier fysiek inactieve) werd deze techniek toegepast en geèvalueerd. De proefpersonen stonden op een pseudo-random motorisch aangedreven platform (rotatie-as gepositioneerd door de enkelgewrichten). De positie en snelheid van het COG werden continue, vrijwel realtime gemeten met het boven vermelde videosysteem. Bij iedere test werd de amplitude van de destabiliserende, pseudorandom platform beweging vergroot zolang de COG snelheid en positie van de proefpersoon zich binnen vooraf ingestelde veilige grenzen bevonden. De vier fysiek zeer actieve proefpersonen tolereerden een grotere platforminstabiliteit dan de drie minder actieve proefpersonen. De vier fysiek inactieve proefpersonen tolereerden slechts een geringe platforminstabiliteit. De test scores correleerden goed met de inschatting die de proefpersonen zelf van de kwaliteit van hun eigen houdingscontrole onder dagelijkse omstandigheden hadden, in tegenstelling tot de routine stabilometrie die geen significante verschillen in lichaamscontrole 
aantoonde (body sway velocity en body sway area). De reproduceerbaarheid van de test scores was beter dan $13.2 \%$.

In hoofdstuk 3 wordt deze nieuwe techniek toegepast en geëvalueerd in 97 gezonde proefpersonen, waarbij tevens het effect van verstoring werd onderzocht op de houdingscontrole door een "sway referenced platform" (SRP) of "sway referenced slide projection" (SRS), het sluiten van de ogen (EC), vilbratie van de achillespezen (VIB) en koelen van de voetzolen (COL). De houdingscontrole nam het meeste af door het sluiten van de ogen $(-37 \%)$ en door vibratie $(-21 \%)$. De andere technieken waren minder effectief. De combinatie van VIB en SRP was het meest effectief om de propriocepsis te verstoren. Het effect van COL was zeer beperkt, mogelijk door een te korte koeltijd ( $5 \mathrm{~min}$.). Over het algemeen was het effect van een combinatie van de perturbatietechnieken geringer dan de lineaire sommatie van het effect van de afzonderlijke technieken. Combinatie van EC, SRP en VIB was het meest effectief en leidde tot een gemiddelde reductie van circa $57 \%$ in houdingscontrole. De data suggereren dat in de huidige stimulus conditie, visuele oriëntatie maximaal $37 \%$, propriocepsis minimaal $26 \%$ en de labyrinten maximaal $44 \%$ bijdragen aan de houdingscontrole in gezonde proefpersonen, en dat afhankelijk van de beschikbare zintuiglijke informatie en de aangeleerde bewegingsstrategieën, de weegfactoren van deze zintuiglijke inputs tussen deze grenzen zouden kunnen variëren. Echter, de individuele data geven aan dat de bijdrage van de zintuigen aan de houdingscontrole sterk varieert per individu en geen generalisatie toelaat, wijzend op een beperkte klinische relevantie van deze vorm van dynamische posturografie.

In hoofdstuk 4 wordt de nieuwe methodiek beproeft om na te gaan of er verschillen. bestaan in houdingscontrole na evenwichtstraining: in een dubbelblind placebo gecontroleerde studie volgden 28 proefpersonen een pseudo-training waar geen effect op de houdingscontrole kon worden verwacht, terwijl 28 proefpersonen de specifieke training op een evenwichtsbalk volgden. Veranderingen in de houdingscontrole werd gemeten met de nieuwe posturografie methode, werden geëvalueerd aan de hand van de prestaties op de evenwichtsbalk zelf, en werden door de proefpersonen zelf geevalueerd aan de hand van de ervaringen bij normale dagelijkse activiteiten. Om het effect van mogelijke habituatie aan de test condities te bepalen werd de houdingscontrole bij $1 \|$ gezonde proefpersonen in drie meet sessies bepaald, met tussenpozen van $\mathbb{1}$ week. Er kon geen significant habituatie effect worden aangetoond. De specifieke training op de evenwichtsbalk toonde een gestage verbetering van de houdingscontrole op de evenwichtsbalk na iedere trainingssessie. Geen der proefpersonen (controle noch training groep) ervoer een verbetering van de houdingscontrole bij de normale dagelijkse activiteiten. Er werd evenmin enig effect gevonden van training op de houdingscontrole gemeten met dynamische posturografie "near the limit of stability". We concluderen dat de nieuwe posturografie methode niet in staat is het effect van een evenwichtstraining te meten, die geen effect heeft op de houdingscontrole bij reguliere dagelijkse activiteiten. In tegenstelling hiermee, werd in hoofdstuk 3 aangetoond dat de mate van houdingscontrole, gemeten met de nieuwe posturografiemethode, wel correspondeert met het eigen oordeel van de proefpersonen over de kwaliteit van de evenwichtsfunctie onder dagelijkse omstandigheden. Wij interpreteren deze bevindingen als dat dynamische posturografie veeleer primair de 
motorische vaardigheden toetst dan het ruimtelijk oriëntatie vermogen noodzakelijk voor een goede houdingscontrole.

In hoofdstuk 5 wordt de nieuwe techniek geèvalueerd bij vier groepen patiënten met langdurige draaiduizeligheid: vijfentwintig Whiplash patiënten, 28 patiënten lijdend aan de ziekte van Meniere, 28 patiënten met persisterende duizeligheid na een eenmalige acuut opgetreden vertigo aanval en 26 patiënten met persisterende duizeligheid bij een langzaam toenemend vestibulair functieverlies. Bij deze patiënten toonde het vestibulair onderzoek afwijkingen bij de calorisatie, het draaistoelonderzoek en de ocular counterrolling geînduceerd door lateroflexie. De houdingscontrole nam het meeste af door het sluiten van de ogen en door vibratie van de achillespezen. De andere technieken waren minder effectief. De combinatie wan VIB en SRP was het meest effectief om de propriocepsis te verstoren. Bij geen enkele patiënten groep werd een consistent specifiek patroon gevonden dat de patiennten naar klachten of het resultaat van het vestibulair onderzoek kon classificeren. De individuele data geven aan dat net als bij gezonde proefpersonen, de bijdrage van de zintuigen aan de houdingscontrole sterk varieert per individu en geen generalisatie toelaat, wijzend op een beperkte klinische relevantie van deze vorm van dynamische posturografie. Echter, bij alle vier patiënten groepen bleek de houdingscontrole wel significant slechter dan bij gezonde proefpersonen, zowel met als zonder zintuiglijke perturbatie. Discriminant analyse toonde dat gebaseerd op de het resultaat van de test condities SRS, EC, SRS+SRP en $\mathrm{EC}+\mathrm{SRP}, 92 \%$ van de patiënten als afwijkend van normaal konden worden geclassificeerd, terwijl alle gezonde proefpersonen als normaal geclassificeerd werden. De discriminantanalyse toonde echter dat de patiënten niet betrouwbaar geclassificeerd konden worden naar de vier onderscheiden patiënten populaties.

In hoofdstuk 6 worden de bewegingsstrategieën geanalyseerd die gebruikt worden voor een goed houdingsevenwicht. Daartoe werden bij zowel gezonde proefpersonen, als bij Whiplash patiënten en patiënten met ziekten passend bij een labyrintair functieverlies hoofd- en lichaamsbewegingen in detail gemeten en geanalyseerd. De gezonde proefpersonen en patiënten bleken gemiddeld allen vergelijkbare snelheden van het massazwaartepunt te hebben, hetgeen op grond wan het feed back karakter van de toegepaste methodiek te verwachten was. Echter de gezonde proefpersonen bleken in staat te zijn de positie en snelheid van het COG te controleren door middel van relatief langzame bewegingen van het hoofd, de romp en de ledematen, terwijl de patiënten veel snellere bewegingen van de lichaamsdelen nodig hadden voor controle van het COG. Tevens bleken de hoeksnelheden van het hoofd bij patienten veel hoger te zijn dan bij de gezonde proefpersonen, hetgeen op een slechtere hoofdstabilisatie of op een actievere rol van hoofdbewegingen bij de houdingscontrole van patienten wijst.

Behoudens dit verschil in hoofdbewegingen, bleek er echter geen verschil in bewegingsstrategie te bestaan tussen patiënten en gezonde proefpersonen, ongeacht de test conditie: er was geen dominantie van een typische heup-, enkel of andere strategie te zien. De lichaamsbewegingen bleken veel complexer te zijn. We stellen daarom voor in plaats van de veel gebezigde termen "heup- of enkelstrategie" door de termen statische en dynamische strategie te vervangen. Onder relatief statische omstandigheden kan het COG binnen de stabiliteitgrenzen gehouden worden door een 
statische strategie waarbij vooral rotatie rond het enkelgewricht plaatsvindt (equivalent aan de "enkelstrategie"). Deze techniek is niet in staat tot snelle houdingscorrecties, maar is energetisch voordelig. Onder dynamische meer complexere omstandigheden, bij een instabiele ondergrond of een klein steunvlak, controleert de mens de positie en snellheid van het $C O G$ door middel van het principe van actie en reactie: snelle bewegingen van lichaamsdelen in een richting leiden tot een verplaatsing van het hele lichaam (en het $\mathrm{COG}$ ) in tegenovergestelde richting. Deze dynamische techniek is snel, maar vergt naast energie ook een relatief grote mate van motorische vaardigheid. Bij deze strategie neemt de snelheid van hoofd, romp en ledematen toe.

In hoofdstuk 7 , wordt in een algemene discussie, de in dit proefschrift geïntroduceerde nieuwe techniek kritisch geèvalueerd. Lopende ons onderzoek werden we ons meer en meer bewust van het feit dat de houdingscontrole een fenomeen is dat sterk afhangt van de precieze stimulus condities, en daarmee de context waaronder het evenwicht bewaard moet worden. Daarmee is extrapolatie van de interpretatie van de in deze studie verkregen gegevens naar die verkregen met stabilometrie of dynamische posturografie "in relatieve rust" maar zeer beperkt mogelijk. De in deze studie gebruikte $4 \mathrm{D}$ techniek heeft ons inziens te veel beperkingen om voldoende inzicht te geven in de houdingscontrole. Met de inmiddels beschikbare video-technieken en andere technologische ontwikkelingen is een $6 \mathrm{D}$ analyse mogelijk waarbij niet alleen de voor-achterwaartse maar ook de zijwaartse lichaamsbewegingen in relatie tot de houdingscontrole zouden kunnen worden bestudeerd.

Op grond van al het bovenstaande komen we tot de conclusie dat de hier gepresenteerde methodiek de houdingscontrole en de invloed van de verschillende zintuigsystemen op de houdingscontrole reproduceerbaar kwantificeert. Een belangrijke conclusie van deze studie is echter wel dat zowel de bewegingsstrategieën toegepast bij de houdingscontrole als de afhankelijkheid van de houdingscontrole van de verschillende zintuigsystemen sterk varieert per individu en dat er over het algemeen geen systematische en typerende verschillen lijken te zijn tussen gezonde mensen en de hier onderzochte patiënten groepen. De conclusie lijkt gerechtvaardigd dat dynamische posturografie, "below" maar ook "near the limit of stability" vooralsnog maar een beperkte klinische relevantie lijkt te hebben. Omdat juist bij dynamische posturografie ook het motorisch controle apparaat een dominante rol speelt bij de mate van houdingscontrole, en een objectiveren van de zintuiglijke bijdrage bemoeilijkt, zal onze research zich in de toekomst primair richten op de bijdrage van de verschillende zintuigsystemen op het ruimtelijk oriëntatie vermogen noodzakelijk voor de houdingscontrolle. 
Acknowledgements 
With the most cordial feeling of thankfulness, I would like to express my deepest appreciation to Prof. dr. J.J. Manni, Professor of Oto-Rhino-Laryngology and Head and Neck Surgery (ORL) of the University of Maastricht, The Netherlands, head of the department, for his acceptance to do this work in his department. I am grateful for his effort to revise this book, his suggestions, his support and encouragement.

I am deeply indebted, grateful and thankful to Dr. Herman Kingma, department of Balance disorders (dept. ORL) of the University of Maastricht, The Netherlands, for giving me the chance to do this work. Because of his precious and unique ideas, valuable guidance and outstanding supervision, this work could be done.

I wish to express my deep thanks to Prof. Dr. Mamdoh EL-Gouhary, Professor of OtoRhino-Laryngology and Head of the Vestibulogy department, Facuity of Medicine, Ain Shams University Hospital, Cairo, Egypt, for giving me the privilege to work in this field.

I. would like to thank Miss Marlies Dolmans, ENG-technician, department of ORL, University of Maastricht, The Netherlands, for her cooperation, support and encouragement. Through her valuable assistance, this work could be done.

My deep gratitude to Iwan De Jong, engineer in technical computer sciences, department of ORL, University of Maastricht, The Netherlands, for his great and indispensable contribution in this work.

I am thankful as well to Harrie Gullikers and Ron Jongen, engineers in technical computer sciences and electrotechnics, University Hospital Mastricht, The Netherlands, for their assistance and friendly support.

I wish to express my gratefulness to Miss Anouk Brandts, secretary of the department, of ORL, University of Maastricht, The Netherlands, for her cooperation and valuable help to start this work.

My deep thanks to Joyce Prins, secretary of the department of ORL, University of Maastricht, The Netherlands, for her tremendous work to finalise this book.

I am thankful to Pauline Kruiniger, secretary of the department of ORL, University of Maastricht, The Netherlands, for her help in this work.

At last, not the least, many thanks to the Faculty of Medicine, University of Maastricht, the University Hospital Maastricht, The Netherlands, for giving me the chance to do this work.

I am also very grateful to everyone who helped me throughout this work. 


\section{Curriculum Vitae}


The author has been born in Cairo, Egypt, on 6 June 1966. He finished his high school studies on 1983 in Cairo. In 1989, he got his M.B.B.Ch., in Medicine and General Surgery. He finished his one year practise as a house officer in Ain Shams University Hospital on March 1991. After that, he worked as a resident of ear, nose and throat in the same university hospital till November 1994. During this period, he wrote an essay and got his master degree in ear, nose and throat specialty. The title of the essay was "The Role of Dynamic Posturography in Neurotological Diagnosis". On December 1994, he was assigned as an assistant lecturer of ear, nose and throat in the faculty of medicine, Ain Shams uniwersity. During this period, he joined the vestibular department of Maastricht University Hospital, The Netherlands, as a research fellow from November 1994 till March 1996. On April 1997, he got his Medical Doctor degree in ear, nose and throat from the faculty of medicine, Ain Shams university, Cairo. On August 1997, he was promoted as a lecturer. 